\title{
Higher spin currents in Wolf space for generic $N$
}

\author{
Changhyun Ahn and Hyunsu Kim \\ Department of Physics, Kyungpook National University, \\ Taegu 702-701, Korea \\ E-mail: ahn@knu.ac.kr, kimhyun@knu.ac.kr
}

ABstract: We obtain the 16 higher spin currents with spins $\left(1, \frac{3}{2}, \frac{3}{2}, 2\right),\left(\frac{3}{2}, 2,2, \frac{5}{2}\right)$, $\left(\frac{3}{2}, 2,2, \frac{5}{2}\right)$ and $\left(2, \frac{5}{2}, \frac{5}{2}, 3\right)$ in the $\mathcal{N}=4$ superconformal Wolf space coset $\frac{\mathrm{SU}(N+2)}{\mathrm{SU}(N) \times \mathrm{SU}(2) \times \mathrm{U}(1)}$. The antisymmetric second rank tensor occurs in the quadratic spin- $\frac{1}{2}$ Kac-Moody currents of the higher spin- 1 current. Each higher spin- $\frac{3}{2}$ current contains the above antisymmetric second rank tensor and three symmetric (and traceless) second rank tensors (i.e. three antisymmetric almost complex structures contracted by the above antisymmetric tensor) in the product of spin- $\frac{1}{2}$ and spin-1 Kac-Moody currents respectively. Moreover, the remaining higher spin currents of spins $2, \frac{5}{2}, 3$ contain the combinations of the (symmetric) metric, the three almost complex structures, the antisymmetric tensor or the three symmetric tensors in the multiple product of the above Kac-Moody currents as well as the composite currents from the large $\mathcal{N}=4$ nonlinear superconformal algebra.

KeYwords: AdS-CFT Correspondence, Conformal and W Symmetry

ARXiv EPRINT: 1411.0356 


\section{Contents}

1 Introduction 1

2 The large $\mathcal{N}=4$ nonlinear superconformal algebra in the Wolf space coset

2.1 The $\mathcal{N}=1$ Kac-Moody current algebra in component approach

2.2 The 11 currents of $\mathcal{N}=4$ nonlinear superconformal algebra using the KacMoody currents

3 The higher spin currents in the Wolf space coset

3.1 One higher spin-1 current

3.2 Four higher spin- $\frac{3}{2}$ currents

3.3 Six higher spin-2 currents

3.4 Four higher spin- $\frac{5}{2}$ currents

3.5 One higher spin-3 current

4 Conclusions and outlook

$\begin{array}{ll}\text { A The generators of } \mathrm{SU}(N+2) \text { in complex basis } & 27\end{array}$

B Eight rank-two tensors in the Wolf space coset $\quad 30$

C The large $\mathcal{N}=4$ nonlinear superconformal algebra 31

D The OPEs between the spin- $\frac{3}{2}$ currents for general $N$ in the bispinor notation

E The identities from the cubic terms in the first-order pole of the OPE between the spin- $\frac{3}{2}$ currents in (2.6)

F Some OPEs and identities relevant to the higher spin- $\frac{5}{2}$ currents $\quad 36$

G Some OPEs generating the higher spin currents (for $N=3,5,7,9$ ) $\quad 37$

H The coefficients and some OPEs relevant to the higher spin-3 current 39

\section{Introduction}

The three-point function in a two-dimensional conformal field theory is an important ingredient which determines the higher point functions via factorization. Another reason to study the three-point function is the fact that the three-point function in two-dimensions 
can provide some information on the three-point function in the three-dimensional bulk theory. In fact, the three-point function shares the same formula in the duality [1-3] between the higher spin theory on the $A d S_{3}$ space and the $W_{N}$ minimal model coset conformal field theory in two-dimensions. The eigenvalue equations (before calculating the three-point function) for the zero modes of the higher spin currents in the coset model match with those of the higher spin fields in the asymptotic symmetry algebra of the higher spin theory on the $A d S_{3}$ space. The simplest three-point function contains two scalar primaries with one higher spin current which is a polynomial combination of the spin-1 WZW affine currents acting on the coset primaries. Also one can study the three-point function in the 'supersymmetric' duality. In [4], the large $\mathcal{N}=4$ higher spin theory on $A d S_{3}$ based on the higher spin algebra is dual to the 't Hooft limit of the two dimensional large $\mathcal{N}=4$ superconformal coset theory on Wolf space [5-7]. Therefore, in order to reveal this duality precisely from three-point function viewpoint, it is necessary, as a first step, to obtain the higher spin currents in Wolf space for general $N$ (and general level $k$ ) which is the main motivation of this paper.

Let us describe the large $\mathcal{N}=4$ superconformal coset theory in two dimensions. The general coset with $\mathrm{U}(M)$ Chan-Paton factor and corresponding Virasoro central charge are described by $[8]$

$$
\frac{G}{H}=\frac{\mathrm{SU}(N+M)_{k} \times \mathrm{SO}(2 N M)_{1}}{\mathrm{SU}(N)_{k+M} \times \mathrm{U}(1)_{N M(N+M)(N+M+k)}}, \quad c=\frac{3 N M k}{(N+M+k)}+\frac{(k+N)\left(M^{2}-1\right)}{(N+M+k)} .
$$

For $M=1$, the coset model (1.1) divided by $\mathrm{SU}(M=1)_{k+N}$ is dual to the $\mathcal{N}=2$ higher spin supergravity from the results in [9] within the context of Kazama-Suzuki model $[10,11]$. In the appropriate 't Hooft limit, the central charge behaves as $N$. For $M=1$, there is no contribution from the second term of the central charge. See also the relevant works in [12-17].

For $M=2$, the above coset (1.1) is dual to the $\mathcal{N}=4$ higher spin supergravity [4]. The central charge behaves as $N$ in the 't Hooft limit. Note that the Virasoro central charge is the sum of Wolf space central charge $c_{\mathrm{W}}=\frac{3 N M k}{(N+M+k)}=\frac{6 N k}{(N+k+2)}$ and the $\mathrm{SU}(M=$ $2)_{k+N}$ central charge. The contributions from the latter appear in each factor group of the coset except $\mathrm{SU}(N)_{k+M}$ group. If one divides the coset by $\mathrm{SU}(M=2)_{k+N}$ further, then one has the Wolf space central charge $c_{\mathrm{W}}$. Once the four spin- $\frac{3}{2}$ currents with an appropriate normalization constant are fixed, then the third-order pole provides the above Wolf space central charge $c_{\mathrm{W}}$ (or the highest order-pole determines the normalization constant) and the Virasoro central charge $c$ in (1.1) is determined automatically from the large $\mathcal{N}=4$ nonlinear superconformal algebra in these four spin- $\frac{3}{2}$ currents. The decomposition of central charge in (1.1) implies that the two stress energy tensors from each part are orthogonal to each other and they commute with each other. We will come to this issue including the stress energy tensor and the four spin- $\frac{3}{2}$ currents in section 2 in detail. From the supersymmetric coset in the abstract to this bosonic coset, one realizes that the $2 N M(=4 N)$ free fermions in $\mathrm{SO}(2 N M)_{1}$ group survive after factoring out the four spin- $\frac{1}{2}$ fermion currents and one spin-1 current living in the large $\mathcal{N}=4$ linear superconformal algebra. The original number of free fermions was given by $\left(2 N M+M^{2}=4 N+4\right)$ which is the same as the bosonic degree of freedoms. 
For general $M(>2)$, the property of the higher spin algebra and the corresponding asymptotic symmetry algebra was described in [18]. See also the work of [19].

See also the relevant works $[20,21]$ on the large $\mathcal{N}=4$ holography [4]. For the particular level at the Kazama-Suzuki model, the $\mathcal{N}=3$ (enhanced) supersymmetry is observed in [22]. This behavior also appeared in previous examples of [23-25].

Before we are going to consider the higher spin currents, let us review on the large $\mathcal{N}=4$ nonlinear superconformal algebra [26-29]. In the work of Van Proeyen [27], the realization of large $\mathcal{N}=4$ nonlinear superconformal algebra was obtained. The four fermionic spin- $\frac{3}{2}$ currents corresponding to $\mathcal{N}=4$ supersymmetries can be associated with the unit matrix and the three complex structures. The former plays the role of $\mathcal{N}=1$ supersymmetry while the latter plays the role of the remaining, additional $\mathcal{N}=3$ supersymmetries. The three components of the spin- 1 currents are related to the three complex structures in the bosonic Kac-Moody currents. The three components of other spin-1 currents are associated with the three complex structures in the product of two fermionic Kac-Moody currents. The spin-2 stress energy tensor can be written in terms of two commuting parts, the stress energy tensor corresponding to Wolf space and the stress energy tensor from the Sugawara construction in the two kinds of above spin-1 currents (the above $\mathrm{SU}(2)_{k+N}$ group describes these two currents where the level is given by $k$ and $N$ respectively) where the index of generators appears nontrivially: although each part differs for each supersymmetry, the sum of these two parts leads to the final spin-2 stress energy tensor. In other words, the four different cases for each part provides an unique expression for the spin-2 stress energy tensor of large $\mathcal{N}=4$ nonlinear superconformal algebra.

Furthermore, in the Gates and Ketov's work [29], the above three complex structures represent almost quaternionic (tri-hermitian) structure on the Wolf space (quaternionic symmetric space) coset. They have considered the nonsymmetric coset space also where the four spin- $\frac{3}{2}$ currents contain the rank three tensor with coset indices and it turned out that one of the spin- 1 currents contain the quadratic terms in the fermionic Kac-moody currents. By using the results about the coset realizations of the large $\mathcal{N}=4$ nonlinear superconformal algebra over the so-called Freudenthal triple systems [30], they identified the above 11 generators with the $\mathcal{N}=4$ WZW generators and the quaternionic structure on the Wolf space can be written in terms of the symplectic structure of the associated Freudenthal triple systems. ${ }^{1}$

\footnotetext{
${ }^{1}$ For the large $\mathcal{N}=4$ linear superconformal algebra [31-37], Sevrin and Theodoridis [38] have determined the 14 currents of the large $\mathcal{N}=4$ linear superconformal algebra from the $\mathcal{N}=1$ super Kac-Moody currents in $\mathcal{N}=1$ superspace. The various tensors appearing in these seven $\mathcal{N}=1$ super fields are satisfied the nontrivial relations. In particular, the three tensors appearing in the quadratic in the $\mathcal{N}=1$ super KacMoody currents of $\mathcal{N}=1$ super field of superspin one play the role of almost complex structures. Of course, the usual $\mathcal{N}=1$ Sugawara stress energy tensor is given. In component approach, in [39], the complete expressions for the 16 currents of large $\mathcal{N}=4$ linear superconformal algebra were written in terms of WZW Kac-Moody currents. For the spin- $\frac{3}{2}$ currents, there are cubic terms in the fermionic WZW Kac-Moody currents with four tensors of rank three. One of them is given by a structure constant and three of them are given by the above almost complex structure contracted with a structure constant. The spin- 1 currents contain the quadratic fermion Kac-Moody currents with almost complex structure contracted two structure constants. Moreover, other spin-1 current has the quadratic fermion Kac-Moody currents with two almost complex structures contracted two structure constants.
} 
Beyond the large $\mathcal{N}=4$ nonlinear (or linear) superconformal algebra, the existence of higher spin currents was observed in [4]. The lowest $\mathcal{N}=4$ higher spin multiplet contains, one spin- 1 current, four spin- $\frac{3}{2}$ currents, six spin- 2 currents, four spin- $\frac{5}{2}$ currents and one spin-3 current. They transform as $(\mathbf{1}, \mathbf{1}),(\mathbf{2}, \mathbf{2}),(\mathbf{1}, \mathbf{3}) \oplus(\mathbf{3}, \mathbf{1}),(\mathbf{2}, \mathbf{2})$ and $(\mathbf{1}, \mathbf{1})$ under the $\mathrm{SU}(2) \times \mathrm{SU}(2)$ respectively. The explicit realization for an extension of large $\mathcal{N}=4$ nonlinear superconformal algebra in the $\mathcal{N}=4$ Wolf space coset theory has been studied in $[40,41]$ for $N=3$. This construction includes the previous descriptions in $[27,29]$. As we vary $N$ starting from $N=3$, the operator product expansions (OPEs) from [40, 41] reveal the $N$-dependence in their structure constants. From the lessons in [40,41], one can determine all the 16 higher spin currents for general $N$ once one obtains the lowest spin-1 current completely with the help of the spin- $\frac{3}{2}$ currents of large $\mathcal{N}=4$ nonlinear superconformal algebra. The metric on the $\mathrm{SU}(N+2)$ group, the three complex structures and structure constants played an important role in the construction of large $\mathcal{N}=4$ nonlinear superconformal algebra and they will appear in the construction of higher spin currents also.

The higher spin-1 current consists of the linear bosonic Kac-Moody current and the quadratic fermionic Kac-Moody currents. One should determine the two coefficient tensors appearing in this higher spin-1 current, along the lines of [40,41], by requiring that this lowest higher spin-1 current transforms as a primary current under the Virasoro stress energy tensor in (1.1) and it does not have any singular terms in the OPE with two SU(2) spin-1 currents of large $\mathcal{N}=4$ nonlinear superconformal algebra.

In this paper, we describe the important aspects in the large $\mathcal{N}=4$ nonlinear superconformal realization considered in $[27,29]$. First of all, one has four spin- $\frac{3}{2}$ currents for $N=3$. We would like to write these four spin- $\frac{3}{2}$ currents for general $N$ because the OPEs between these currents give all the information on the remaining seven currents, six spin-1 currents and one spin-2 current. All the 16 OPEs have the explicit singular terms in terms of spin-1 and spin- $\frac{1}{2}$ Kac-Moody currents. As observed in [27], one observes the three complex structures $h_{\bar{a} \bar{b}}^{i}$ in front of the above spin- $\frac{3}{2}$ currents in terms of $4 N \times 4 N$ matrices where the $4 N$ is the number of coset indices. These complex structures also appear in the spin-1 currents. One can read off the spin-2 stress energy tensor from the first-order pole in the some OPEs among the above 16 OPEs.

As emphasized before, the higher spin-1 current contains the antisymmetric second rank tensor $d_{\bar{a} \bar{b}}^{0}$ with the Wolf space coset indices in front of the quadratic fermionic KacMoody currents. This antisymmetric second rank tensor is a new quantity and satisfies the nontrivial identities, 1) the square of this tensor gives a metric on the Wolf space coset and 2 ) there exists an identity for the product between this tensor with the structure constant. By construction, the four higher spin- $\frac{3}{2}$ currents contain the product of the three complex structures (including the unit matrix) and the above antisymmetric second rank tensor. The higher spin- 2 currents contain the combinations of the (symmetric) metric, the three almost complex structures, the antisymmetric tensor or the three symmetric tensors in the multiple product of the above Kac-Moody currents as well as the composite currents from the large $\mathcal{N}=4$ nonlinear superconformal algebra. Moreover, the remaining higher spin currents of spins $\frac{5}{2}, 3$ contain more higher rank tensors. For the higher spin currents with spins $s=1, \frac{3}{2}$ and 2, the explicit manifest $\mathrm{SU}(2) \times \mathrm{SU}(2)$ representations are given. 
In section 2, we review the large $\mathcal{N}=4$ nonlinear superconformal algebra. The starting point is to begin with the four spin- $\frac{3}{2}$ currents which determine all the remaining currents (six spin-1 currents and one spin-2 current) in the large $\mathcal{N}=4$ nonlinear algebra.

In section 3, the 16 higher spin currents in terms of bosonic and fermionic Kac-Moody currents are constructed. Some higher spin currents appeared in [20] are identified. The various tensors appearing in the higher spin currents are constructed. Because these higher spin currents are constructed in terms of Kac-Moody currents, the zero modes can be obtained.

In section 4, we summarize the results of this paper and future directions are given.

In appendices $\mathrm{A}-\mathrm{H}$, some detailed calculations are described.

The mathematica package by Thielemans [42] is used for low $N$ cases with $N=$ $3,5,7,9$. For these values, one obtains all the higher spin currents which are not present in this paper explicitly. They can be obtained from the results of section 3 by substituting these values.

\section{The large $\mathcal{N}=4$ nonlinear superconformal algebra in the Wolf space coset}

In this section, we review the large $\mathcal{N}=4$ nonlinear superconformal algebra in the Wolf space coset. The 11 currents are described in terms of the $\mathcal{N}=1$ Kac-Moody currents.

\subsection{The $\mathcal{N}=1$ Kac-Moody current algebra in component approach}

Let us consider the group $\mathrm{SU}(N+2)$ in the Wolf space coset where $N$ is odd and the dimension of $\mathrm{SU}(N+2)$ is even. The generators are given in appendix A. They satisfy the usual commutation relation $\left[T_{a}, T_{b}\right]=f_{a b}{ }^{c} T_{c}$ where the indices run over $a, b, \cdots=$ $1,2, \cdots,(N+2)^{2}-1$. The normalization is as follows: $g_{a b}=\frac{1}{2 c_{G}} f_{a c}{ }^{d} f_{b d}{ }^{c}$ where $c_{G}$ is the dual Coxeter number of the group $c_{\mathrm{SU}(N+2)}=(N+2)$. Let us denote the inverse of the metric as $g^{a b} \equiv g_{a b}^{-1}$.

The operator product expansions between the spin- 1 and the spin- $\frac{1}{2}$ currents are described as [43]

$$
\begin{aligned}
& V^{a}(z) V^{b}(w)=\frac{1}{(z-w)^{2}} k g^{a b}-\frac{1}{(z-w)} f_{c}^{a b} V^{c}(w)+\cdots, \\
& Q^{a}(z) Q^{b}(w)=-\frac{1}{(z-w)}(k+N+2) g^{a b}+\cdots \\
& V^{a}(z) Q^{b}(w)=+\cdots
\end{aligned}
$$

Here $k$ is the level and a positive integer. Note that there is no singular term in the OPE between the spin-1 current $V^{a}(z)$ and the spin- $\frac{1}{2}$ current $Q^{b}(w)$. One can also obtain these OPEs in the $\mathcal{N}=1$ superspace. Furthermore, the $\mathcal{N}=2$ superspace description where the spin-1 currents have the additional quadratic fermionic terms can be obtained by introducing some nonlinear constraints as in [44]. Note that the first OPE in (2.1) has three independent OPEs if one uses the notations (with bar and unbarred indices) in $[40,41]$. 


\subsection{The 11 currents of $\mathcal{N}=4$ nonlinear superconformal algebra using the Kac-Moody currents}

As in the abstract, the Wolf space coset in the 'supersymmetric' version is as follows:

$$
\text { Wolf }=\frac{G}{H}=\frac{\mathrm{SU}(N+2)}{\mathrm{SU}(N) \times \mathrm{SU}(2) \times \mathrm{U}(1)} .
$$

Let us denote the indices belonging to $G$ and Wolf space coset $\frac{G}{H}$ as

$$
\begin{aligned}
& G \quad \text { indices : } a, b, c, \cdots=1,2, \cdots,(N+2)^{2}-1, \\
& \frac{G}{H} \quad \text { indices : } \bar{a}, \bar{b}, \bar{c}, \cdots=1,2, \cdots, 4 N .
\end{aligned}
$$

One can also introduce the subgroup $H$ indices but does not present them because they do not appear in later expressions. ${ }^{2}$ For given $(N+2) \times(N+2)$ matrix, one can associate the $4 N$ coset indices as follows:

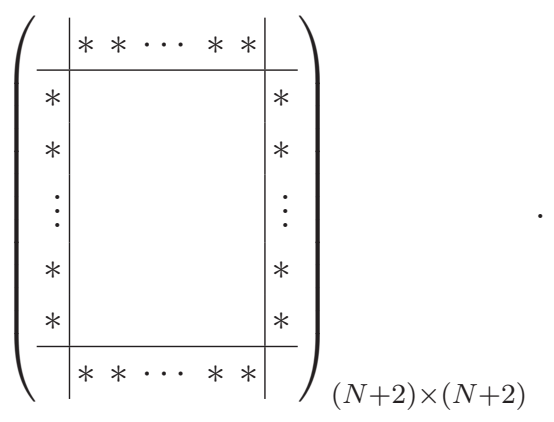

For example, the Wolf space coset generators are given in appendix A. The $N \times N$ matrix corresponding to the subgroup $\mathrm{SU}(N)$ in (2.2) is located at the inside of (2.4).

Let us start with the four fermionic spin- $\frac{3}{2}$ currents. From the $N=3$ result in [40], one can write the spin- $\frac{3}{2}$ currents $G^{\mu}(z)$ where an index $\mu$ is in the $\mathrm{SO}(4)$ vector representation as follows: ${ }^{3}$

$$
G^{\mu}(z)=A(k, N) h_{\bar{a} \bar{b}}^{\mu} Q^{\bar{a}} V^{\bar{b}}(z), \quad \mu=0,1,2,3,
$$

where $h_{\bar{a} \bar{b}}^{0} \equiv g_{\bar{a} \bar{b}}$ and $h_{\bar{a} \bar{b}}^{i}(i=1,2,3)$ are numerical constants and the $A(k, N)$ is normalization factor to be determined. Note that the indices appearing in (2.5) belong to the Wolf space coset. There is no difference in the ordering of two different currents $Q^{\bar{a}}(z)$ and $V^{\bar{b}}(z)$ because they do not have any singular terms as mentioned before. By calculating the OPE $G^{\mu}(z) G^{\nu}(w)$ which should satisfy the $\mathcal{N}=4$ nonlinear superconformal algebra (C.2) (or (C.4)), one should obtain the other currents in the right hand side of the OPE as well as the normalization constant in (2.5).

\footnotetext{
${ }^{2}$ In [40], one introduced the complex basis where the indices are given by barred and unbarred ones. In this paper, one uses the different notations. The indices $a, b, \cdots$ are given by $A$ and $A^{*}$ while the indices $\bar{a}, \bar{b}, \cdots$ are given by $\bar{A}$ and $\bar{A}^{*}$ in the complex basis. See also appendix A.

${ }^{3}$ One can assume more general ansatz which has cubic fermionic terms [29]. But in the Wolf space coset, the rank three tensor in front of these terms vanishes.
} 
Let us compute the OPE $G^{\mu}(z) G^{\nu}(w)$ using the basic OPEs in (2.1). See also the relevant works in [45-47]. It turns out that

$$
\begin{aligned}
G^{\mu}(z) G^{\nu}(w)= & \frac{1}{(z-w)^{3}} A^{2}\left[-k(k+N+2) h_{\bar{a} \bar{b}}^{\mu} h^{\nu \bar{a} \bar{b}}\right] \\
& +\frac{1}{(z-w)^{2}} A^{2}\left[(k+N+2) h_{\bar{a} \bar{b}}^{\mu} h^{\nu \bar{a}} f_{e}^{\bar{b} \bar{d}} V^{e}+k h_{\bar{a} \bar{b}}^{\mu} h_{\bar{c}}^{\nu \bar{b}} Q^{\bar{a}} Q^{\bar{c}}\right](w) \\
& +\frac{1}{(z-w)} A^{2}\left[-(k+N+2) h_{\bar{a} \bar{b}}^{\mu} h^{\nu \bar{a}} V_{\bar{d}}^{\bar{b}} V^{\bar{d}}+k h_{\bar{a} \bar{b}}^{\mu} h_{\bar{c}}^{\nu \bar{b}} \partial Q^{\bar{a}} Q^{\bar{c}}\right. \\
& \left.-h_{\bar{a} \bar{b}}^{\mu} h_{\bar{c} \bar{d}}^{\nu} f_{e}^{\bar{b} \bar{d}} Q^{\bar{a}} Q^{\bar{c}} V^{e}\right](w)+\cdots .
\end{aligned}
$$

Note that the spin-1 current contracted with the structure constant in (2.6) contains the $\mathrm{SU}(N+2)$ group index. The normalization constant $A(k, N)$ is determined by the Wolf space central charge term and one takes $\mu=\nu=0$ and $\left.G^{0}(z) G^{0}(w)\right|_{\frac{1}{(z-w)^{3}}}=\frac{2}{3} c_{\text {Wolf }}$ where the Wolf space coset central charge is given by $c_{\text {Wolf }}=\frac{6 k N}{(2+k+N)}$ in (1.1). This implies that $A(k, N)=\frac{i}{(k+N+2)}$ in (2.5) where we used $g_{\bar{a} \bar{b}} g^{\bar{a} \bar{b}}=4 N$. Therefore, the four spin- $\frac{3}{2}$ currents are given by

$$
\begin{aligned}
G^{0}(z) & =\frac{i}{(k+N+2)} Q_{\bar{a}} V^{\bar{a}}(z), \\
G^{i}(z) & =\frac{i}{(k+N+2)} h_{\bar{a} \bar{b}}^{i} Q^{\bar{a}} V^{\bar{b}}(z), \quad(i=1,2,3) .
\end{aligned}
$$

These equations correspond to the equation (3.18) of [29]. ${ }^{4}$

Let us describe the OPE structure (2.6) in detail. Let us focus on the quadratic spin-1 currents which appear in the first term in the first-order pole in (2.6). One has similar term for the OPE $G^{\nu}(z) G^{\mu}(w)$. The sum of these two terms should be equal to the twice of stress energy term (C.2) which contains also quadratic spin-1 currents as follows:

$$
h_{\bar{a} \bar{b}}^{\mu} h_{\bar{d}}^{\nu \bar{a}}+h_{\bar{a} \bar{b}}^{\nu} h_{\bar{d}}^{\mu \bar{a}}=2 \delta^{\mu \nu} g_{\bar{b} \bar{d}}, \quad(\mu, \nu=0,1,2,3) .
$$

$$
\begin{aligned}
& { }^{4} \text { For } N=3 \text {, one has the following results with (2.16) from [40]: } \\
& G^{0}(z)=\frac{i}{(5+k)}\left(\sum_{\left(\bar{A}, \bar{A}^{*}\right)=\left(\overline{1}, \overline{1}^{*}\right)}^{\left(\overline{3}, \overline{3}^{*}\right)} Q^{\bar{A}} V^{\bar{A}^{*}}+\sum_{\left(\bar{A}^{*}, \bar{A}\right)=\left(\overline{1}^{*}, \overline{1}\right)}^{\left(\overline{3}^{*}, \overline{3}\right)} Q^{\bar{A}^{*}} V^{\bar{A}}+\sum_{\left(\bar{A}, \bar{A}^{*}\right)=\left(\overline{4}, \bar{A}^{*}\right)}^{\left(\overline{6}, \overline{6}^{*}\right)} Q^{\bar{A}} V^{\bar{A}^{*}}+\sum_{\left(\bar{A}^{*}, \bar{A}\right)=\left(\bar{A}^{*}, \overline{4}\right)}^{\left(\overline{6}^{*}, \overline{6}\right)} Q^{\bar{A}^{*}} V^{\bar{A}}\right)(z), \\
& G^{1}(z)=\frac{i}{(5+k)}\left(\sum_{(\bar{A}, \bar{B})=(\overline{4}, \overline{1})}^{(\overline{6}, \overline{3})} Q^{\bar{A}} V^{\bar{B}}+\sum_{\left(\bar{A}^{*}, \bar{B}^{*}\right)=\left(\overline{4} \overline{4}^{*}, \overline{1}^{*}\right)}^{\left(\overline{6}^{*}, \overline{3}^{*}\right)} Q^{\bar{A}^{*}} V^{\bar{B}^{*}}-\sum_{(\bar{A}, \bar{B})=(\overline{1}, \overline{4})}^{(\overline{3}, \overline{6})} Q^{\bar{A}} V^{\bar{B}}-\sum_{\left(\bar{A}^{*}, \bar{B}^{*}\right)=\left(\overline{1}^{*}, \overline{4}^{*}\right)}^{\left(\overline{3}^{*}, \overline{6}^{*}\right)} Q^{\bar{A}^{*}} V^{\bar{B}^{*}}\right)(z), \\
& G^{2}(z)=\frac{1}{(5+k)}\left(-\sum_{(\bar{A}, \bar{B})=(\overline{4}, \overline{1})}^{(\overline{6}, \overline{3})} Q^{\bar{A}} V^{\bar{B}}+\sum_{\left(\bar{A}^{*}, \bar{B}^{*}\right)=\left(\bar{A}^{*}, \overline{1}^{*}\right)}^{\left(\overline{6}^{*}, \overline{3}^{*}\right)} Q^{\bar{A}^{*}} V^{\bar{B}^{*}}+\sum_{(\bar{A}, \bar{B})=(\overline{1}, \overline{4})}^{(\overline{3}, \overline{6})} Q^{\bar{A}} V^{\bar{B}}-\sum_{\left(\bar{A}^{*}, \bar{B}^{*}\right)=\left(\overline{1}^{*}, \bar{A}^{*}\right)}^{\left(\overline{3}^{*}, \overline{6}^{*}\right)} Q^{\bar{A}^{*}} V^{\bar{B}^{*}}\right)(z), \\
& G^{3}(z)=\frac{1}{(5+k)}\left(-\sum_{\left(\bar{A}, \bar{A}^{*}\right)=\left(\bar{A}, \bar{A}^{*}\right)}^{\left(\overline{6}, \overline{6}^{*}\right)} Q^{\bar{A}} V^{\bar{A}^{*}}+\sum_{\left(\bar{A}^{*}, \bar{A}\right)=\left(\bar{A}^{*}, \overline{4}\right)}^{\left(\overline{6}^{*}, \bar{b}\right)} Q^{\bar{A}^{*}} V^{\bar{A}}-\sum_{\left(\bar{A}, \bar{A}^{*}\right)=\left(\overline{1}, \overline{1}^{*}\right)}^{\left(\overline{3}, \overline{3}^{*}\right)} Q^{\bar{A}} V^{\bar{A}^{*}}+\sum_{\left(\bar{A}^{*}, \bar{A}\right)=\left(\overline{1}^{*}, \overline{1}\right)}^{\left(\overline{3}^{*}, \overline{3}\right)} Q^{\bar{A}^{*}} V^{\bar{A}}\right)(z),
\end{aligned}
$$

which is consistent with the ones in (2.7) with the help of (B.1). Here the indices 10,11, 12 (and their complex conjugated ones) in [40] are replaced by 4,5,6 (and their conjugated ones) and the indices 7,8 (and their complex conjugated ones) are replaced by 11, 12 (their complex conjugated ones). The remaining indices can be identified each other. The Wolf space coset indices in this case are given by $(\overline{1}, \overline{2}, \overline{3})$ and $(\overline{4}, \overline{5}, \overline{6})$ (and their complex conjugated ones). 
This equation (2.8) corresponds to the equation (3.6) of [29]. One can obtain two properties from (2.8) as follows:

$$
\begin{aligned}
h_{\bar{a} \bar{b}}^{i} & =-h_{\bar{b} \bar{a}}^{i}, \\
h_{\bar{a} \bar{b}}^{i} h_{\bar{c}}^{i \bar{a}} & =g_{\bar{b} \bar{c}},
\end{aligned}
$$

where $i=1,2,3$ and there is no sum over the index $i$ in the second equation of (2.9). The first one in (2.9) corresponds to the case $\mu=i$ and $\nu=0$ while the second one in (2.9) corresponds to the case $\mu=\nu=i$ in (2.8). The complex structure is antisymmetric in the Wolf space coset indices according to the first equation of (2.9). The conditions in (2.9) imply that the $h^{i}$ are almost complex structures and correspond to the equations (3.8) and (3.9) of [29]. If one defines the third complex structure as

$$
h_{\bar{a} \bar{b}}^{3} \equiv h_{\bar{a} \bar{c}}^{1} h_{\bar{b}}^{2 \bar{c}},
$$

then the three almost complex structures $\left(h^{1}, h^{2}, h^{3}\right)$ satisfy the following relation

$$
h_{\bar{a} \bar{c}}^{i} h^{j \bar{c}}=\epsilon^{i j k} h_{\bar{a} \bar{b}}^{k}-\delta^{i j} g_{\bar{a} \bar{b}} .
$$

This relation corresponds to the quaternionic algebra (2.24) of [39]. See also [48] where the relation (2.11) was described.

For the identities from the cubic terms in (2.6), one presents in appendix E.

Now one should determine other remaining currents. We can identify six spin-1 currents $A^{ \pm i}(z)$ of $\mathrm{SU}(2)_{\hat{k}^{+}} \times \mathrm{SU}(2) \hat{k}^{-}$using the last two equations in (C.4) as follows: ${ }^{5}$

$$
\begin{aligned}
& A^{+i}(z)=-\frac{1}{4 N} f_{c}^{\bar{a} \bar{b}} h_{\bar{a} \bar{b}}^{i} V^{c}(z), \\
& A^{-i}(z)=-\frac{1}{4(k+N+2)} h_{\bar{a} \bar{b}}^{i} Q^{\bar{a}} Q^{\bar{b}}(z), \quad(i=1,2,3) .
\end{aligned}
$$

These equations (2.12) correspond to the equation (3.18) of [29]. They satisfy the follow-

$$
\begin{aligned}
& { }^{5} \text { For } N=3 \text {, the results from [40] with (2.16) lead to the following expressions } \\
& A^{+1}(z)=-\frac{1}{2}\left(-V^{9}+V^{9^{*}}\right)(z), \quad A^{+2}(z)=\frac{i}{2}\left(V^{9}+V^{9^{*}}\right)(z), \\
& A^{+3}(z)=-\frac{1}{12} i(\sqrt{3}+3 i) V^{11}(z)-\frac{i(3 \sqrt{5}+i \sqrt{3})}{12 \sqrt{2}} V^{12}(z)-\frac{1}{12}(3+i \sqrt{3}) V^{11^{*}}(z)-\frac{(\sqrt{3}+3 i \sqrt{5})}{12 \sqrt{2}} V^{12^{*}}(z), \\
& A^{-1}(z)=\frac{1}{2(k+5)}\left(\sum_{(\bar{A}, \bar{B})=(\overline{1}, \overline{4})}^{(\overline{3}, \overline{6})} Q^{\bar{A}} Q^{\bar{B}}+\sum_{\left(\bar{A}^{*}, \bar{B}^{*}\right)=\left(\overline{1}^{*}, \bar{A}^{*}\right)}^{\left(\overline{3}^{*}, \overline{\bar{G}}^{*}\right)} Q^{\bar{A}^{*}} Q^{\bar{B}^{*}}\right)(z), \\
& A^{-2}(z)=\frac{i}{2(k+5)}\left(\sum_{(\bar{A}, \bar{B})=(\overline{1}, \overline{4})}^{(\overline{3}, \overline{6})} Q^{\bar{A}} Q^{\bar{B}}-\sum_{\left(\bar{A}^{*}, \bar{B}^{*}\right)=\left(\overline{1}^{*}, \bar{A}^{*}\right)}^{\left(\overline{3}^{*}, \overline{\bar{b}}^{*}\right)} Q^{\bar{A}^{*}} Q^{\bar{B}^{*}}\right)(z), \\
& A^{-3}(z)=-\frac{i}{2(k+5)}\left(\sum_{\left(\bar{A}, \bar{A}^{*}\right)=\left(\overline{4}, \bar{A}^{*}\right)}^{\left(\overline{6}, \overline{6}^{*}\right)} Q^{\bar{A}} Q^{\bar{A}^{*}}+\sum_{\left(\bar{A}, \bar{A}^{*}\right)=\left(\overline{1}, \overline{1}^{*}\right)}^{\left(\overline{3}, \overline{3}^{*}\right)} Q^{\bar{A}} Q^{\bar{A}^{*}}\right)(z),
\end{aligned}
$$

which is consistent with the ones in (2.12). For the $A^{+i}(z)$ current, it is not obvious to see the consistency because there exists a structure constant in (2.12). However, one can check by looking at the explicit values of the nonzero structure constants in [40]. 
ing $\mathrm{OPEs}^{6}$

$$
A^{ \pm i}(z) A^{ \pm j}(w)=-\frac{1}{(z-w)^{2}} \frac{1}{2} \delta^{i j} \hat{k}^{ \pm}+\frac{1}{(z-w)} \epsilon^{i j k} A^{ \pm k}(w)+\cdots,
$$

where $i, j=1,2,3$ and $\hat{k}^{+}=k$ is the level for $\mathrm{SU}(2)_{\hat{k}^{+}}$while $\hat{k}^{-}=N$ is the level for $\mathrm{SU}(2)_{\hat{k}^{-}}$. They commute with each other because the spin- 1 currents and the spin- $\frac{1}{2}$ currents do not have any singular terms from (2.1). One can easily construct the sum of these two currents $\left(A^{+i}+A^{-i}\right)(z)$ which has the level $\left(\hat{k}^{+}+\hat{k}^{-}\right)=(k+N)$ for $\mathrm{SU}(2)_{\hat{k}^{+}+\hat{k}^{-}} \cdot{ }^{7}$

There are many ways to extract the correct stress energy tensor. For example, one can identify the stress energy tensor from the first-order pole term of $G^{0}(z) G^{0}(w)$. After substituting $\mu=\nu=0$ in both sides of (2.6) and subtracting the correct nonlinear terms coming from the two kinds of spin-1 currents, one arrives at the following result

$$
\begin{aligned}
L(z)= & \frac{1}{2(k+N+2)^{2}}\left[(k+N+2) V_{\bar{a}} V^{\bar{a}}+k Q_{\bar{a}} \partial Q^{\bar{a}}+f_{\bar{a} \bar{b} c} Q^{\bar{a}} Q^{\bar{b}} V^{c}\right](z) \\
& -\frac{1}{(k+N+2)} \sum_{i=1}^{3}\left(A^{+i}+A^{-i}\right)^{2}(z) .
\end{aligned}
$$

The first line in (2.14) corresponds to the right hand side of (2.6) and the second line corresponds to the above nonlinear terms which are the usual Sugawara construction for the above $\mathrm{SU}(2)_{\hat{k}^{+}+\hat{k}^{-}}$currents. The above 10 currents $G^{\mu}(w)$ and $A^{ \pm i}(w)$ are primary under this stress energy tensor $L(z)$ and furthermore one can check the following OPE from the OPEs in $(2.1)^{8}$

$$
L(z) L(w)=\frac{1}{(z-w)^{4}} \frac{\hat{c}}{2}+\frac{1}{(z-w)^{2}} 2 L(w)+\frac{1}{(z-w)} \partial L(w)+\cdots,
$$

where the central charge $\hat{c}=\frac{3(k+N+2 k N)}{(k+N+2)}$ is equal to the one in (1.1).

\footnotetext{
${ }^{6}$ Actually, we have checked that for the upper case, using the currents for low values of $N=3,5,7,9$, this equation holds and for the lower case, one identifies this equation using the property of complex structures.

${ }^{7}$ One can read off the three almost complex structures of $\mathrm{SU}(N+2=5)$ from the spin-1 currents in $(2.12)$ (or the $\hat{B}_{i}(z)$ in [40]) and they are $12 \times 12$ matrices as follows:

$$
h_{\bar{a} \bar{b}}^{1}=\left(\begin{array}{cccc}
0 & -1 & 0 & 0 \\
1 & 0 & 0 & 0 \\
0 & 0 & 0 & -1 \\
0 & 0 & 1 & 0
\end{array}\right), \quad h_{\bar{a} \bar{b}}^{2}=\left(\begin{array}{cccc}
0 & -i & 0 & 0 \\
i & 0 & 0 & 0 \\
0 & 0 & 0 & i \\
0 & 0 & -i & 0
\end{array}\right), \quad h_{\bar{a} \bar{b}}^{3}=h_{\bar{a} \bar{c}}^{1} h^{2 \bar{c}} \bar{b}
$$

where each element is $3 \times 3$ matrix. The $h_{\bar{a} \bar{b}}^{3}$ was defined in (2.10) and three complex structures satisfy (2.11). In appendix $\mathrm{B}$, the $N$-generalization for the complex structures is given.

${ }^{8}$ Also in this case one checked this $\mathrm{OPE}$ for low values for $N=3,5,7,9$. Furthermore, one has the second line of (2.14) as

$$
\begin{aligned}
\sum_{i=1}^{3}\left(A^{+i}+A^{-i}\right)^{2}(z)= & \sum_{i=1}^{3}\left(\frac{1}{16 N^{2}} f^{\bar{a} \bar{b}} h_{\bar{a} \bar{b}}^{i} f_{f}^{\bar{d} \bar{e}} h_{\bar{d} \bar{e}}^{i} V^{c} V^{f}+\frac{1}{8 N(k+N+2)} f^{\bar{a} \bar{b}} h_{\bar{a} \bar{b}}^{i} h_{\bar{d} \bar{e}}^{i} Q^{\bar{d}} Q^{\bar{e}} V^{c}\right. \\
& \left.+\frac{1}{16(k+N+2)^{2}} h_{\bar{a} \bar{b}}^{i} h_{\bar{c} \bar{d}}^{i} Q^{\bar{a}} Q^{\bar{b}} Q^{\bar{c}} Q^{\bar{d}}\right)(z)-\frac{3}{4(k+N+2)} Q_{\bar{a}} \partial Q^{\bar{a}}(z)
\end{aligned}
$$

Note that the spin-1 Kac-Moody current and the spin- $\frac{1}{2}$ Kac-Moody current commute with each other and there is no ordering problem between them. For the expression of $\left(A^{-i} A^{-i}\right)(z)$, one should be careful about the ordering [47]. If one simplifies (2.14) further, then all the expressions can be combined with the ones in the first line of (2.14) except the quartic fermionic term and the quadratic spin-1 currents.
} 
Summarizing this subsection, the large $\mathcal{N}=4$ nonlinear superconformal algebra in terms of the Wolf space coset currents is characterized by (2.6), where the right hand side should be written in terms of the 11 currents, (2.13) and (2.15). Of course, as above, the 10 currents are primary. We are left with the OPEs between the spin-1 currents $A^{ \pm i}(z)$ and the spin- $\frac{3}{2}$ currents $G^{\mu}(w)$. They appear in appendix C. The role of three almost complex structures was very crucial. Again the 11 currents are given by (2.7), (2.12) and (2.14). Due to the nonlinear terms in (2.6), the spin-2 stress energy tensor takes the form of very nontrivial expression. This is the reason why some of the literatures do not have the correct and explicit form for the stress energy tensor. We will explain this feature in appendix C in detail. ${ }^{9}$

\section{The higher spin currents in the Wolf space coset}

Let us recall that the lowest 16 higher spin currents have the following $\mathcal{N}=2$ multiplets with spin contents

$$
\begin{aligned}
& \left(1, \frac{3}{2}, \frac{3}{2}, 2\right):\left(T^{(1)}, T_{+}^{\left(\frac{3}{2}\right)}, T_{-}^{\left(\frac{3}{2}\right)}, T^{(2)}\right), \\
& \left(\frac{3}{2}, 2,2, \frac{5}{2}\right):\left(U^{\left(\frac{3}{2}\right)}, U_{+}^{(2)}, U_{-}^{(2)}, U^{\left(\frac{5}{2}\right)}\right), \\
& \left(\frac{3}{2}, 2,2, \frac{5}{2}\right):\left(V^{\left(\frac{3}{2}\right)}, V_{+}^{(2)}, V_{-}^{(2)}, V^{\left(\frac{5}{2}\right)}\right), \\
& \left(2, \frac{5}{2}, \frac{5}{2}, 3\right):\left(W^{(2)}, W_{+}^{\left(\frac{5}{2}\right)}, W_{-}^{\left(\frac{5}{2}\right)}, W^{(3)}\right) .
\end{aligned}
$$

In this section, we would like to construct these higher spin currents explicitly. As described in the introduction, it is very crucial to obtain the lowest higher spin-1 current in (3.1).

\footnotetext{
${ }^{9}$ One has the explicit relations between the 11 currents in [40] and the ones in this paper as follows:

$$
\begin{aligned}
& \hat{B}_{i}(z)=A^{-i}(z), \quad(i=1,2,3), \\
& \hat{A}_{1}(z)=-A^{+1}(z), \quad \hat{A}_{2}(z)=A^{+2}(z), \quad \hat{A}_{3}(z)=-A^{+3}(z), \\
& \hat{G}_{11}(z)=\frac{1}{\sqrt{2}}\left(G^{1}-i G^{2}\right)(z), \quad \hat{G}_{12}(z)=-\frac{1}{\sqrt{2}}\left(G^{3}-i G^{0}\right)(z), \\
& \hat{G}_{22}(z)=\frac{1}{\sqrt{2}}\left(G^{1}+i G^{2}\right)(z), \quad \hat{G}_{21}(z)=-\frac{1}{\sqrt{2}}\left(G^{3}+i G^{0}\right)(z), \\
& \hat{T}(z)=L(z) .
\end{aligned}
$$

One can calculate the first order pole of the OPE $\hat{G}_{11}(z) \hat{G}_{22}(w)$ using (2.16) and (2.6) and read off the following quantity

$$
\begin{aligned}
\hat{T}(z)= & \frac{1}{2(k+N+2)^{2}}\left[(k+N+2)\left(g-i h^{3}\right)_{\bar{a} \bar{b}} V^{\bar{a}} V^{\bar{b}}+k\left(g+i h^{3}\right)_{\bar{a} \bar{b}} Q^{\bar{a}} \partial Q^{\bar{b}}\right. \\
& \left.+\frac{1}{2}\left(h^{1}-i h^{2}\right)_{\bar{a} \bar{b}}\left(h^{1}+i h^{2}\right)_{\bar{c} \bar{d}} f^{\bar{b} \bar{d}}{ }_{e} Q^{\bar{a}} Q^{\bar{c}} V^{e}\right](z)-\frac{1}{(k+N+2)}\left[i \partial\left(N \hat{A}_{3}-k \hat{B}_{3}\right)\right. \\
& \left.+\frac{1}{2}\left(\hat{A}_{+} \hat{A}_{-}+\hat{A}_{-} \hat{A}_{+}\right)+\hat{A}_{3} \hat{A}_{3}+2 \hat{A}_{3} \hat{B}_{3}+\frac{1}{2}\left(\hat{B}_{+} \hat{B}_{-}+\hat{B}_{-} \hat{B}_{+}\right)+\hat{B}_{3} \hat{B}_{3}\right](z),
\end{aligned}
$$

which corresponds to the equation (4.31) of [49]. In this calculation the properties of complex structures are used and $\hat{A}_{ \pm}(z) \equiv \hat{A}_{1} \pm i \hat{A}_{2}(z)$ and $\hat{B}_{ \pm}(z) \equiv \hat{B}_{1} \pm i \hat{B}_{2}(z)$. 
Once this is found, then it is straightforward to obtain the remaining 15 higher spin currents with the help of the four spin- $\frac{3}{2}$ currents.

\subsection{One higher spin-1 current}

From the $N=3$ result in [40], one expresses the higher spin- 1 current as

$$
\begin{aligned}
T^{(1)}(z)= & -\frac{1}{(k+5)}\left[\frac{5(\sqrt{3}+3 i)}{6} V^{11}-\frac{(9 \sqrt{5}-5 i \sqrt{3})}{6 \sqrt{2}} V^{12}+\frac{5(\sqrt{3}-3 i)}{6} V^{11^{*}}\right. \\
& \left.-\frac{(9 \sqrt{5}+5 i \sqrt{3})}{6 \sqrt{2}} V^{12^{*}}\right](z)+\frac{k}{(k+5)^{2}}\left[\sum_{\left(\bar{A}, \bar{A}^{*}\right)=\left(\overline{1}, \overline{1}^{*}\right)}^{\left(\overline{3} \overline{3}^{*}\right)} Q^{\bar{A}} Q^{\bar{A}^{*}}-\sum_{\left(\bar{A}, \bar{A}^{*}\right)=\left(\overline{6}, \overline{4}^{*}\right)}^{(3.2)} Q^{\bar{A}} Q^{\bar{A}^{*}}\right](z) .
\end{aligned}
$$

How does one generalize this higher spin- 1 current for general $N$ ? For the quadratic terms in (3.2), one can easily see the generalization by expanding the corresponding indices to the $N$ and $N$ respectively. The second summation has an opposite sign. However, for the first four terms in (3.2), one cannot see the behavior for the general $N$ easily because the structure constants in $\mathrm{SU}(5)$ are rather complicated. Based on (3.2), one can try to write the following quantity

$$
T^{(1)}(z)=-\frac{1}{2(k+N+2)} A_{a} V^{a}(z)+\frac{k}{2(k+N+2)^{2}} d_{\bar{a} \bar{b}}^{0} Q^{\bar{a}} Q^{\bar{b}}(z),
$$

where $A_{a}$ and $d_{\bar{a} \bar{b}}^{0}$ are numerical constants (to be determined later) and the $d_{\bar{a} \bar{b}}^{0}$ is antisymmetric by construction. Recall that from the explicit form for the spin-1 currents in previous section in (2.12), one sees the linear $V^{a}(z)$ term and the quadratic $Q^{\bar{a}} Q^{\bar{b}}(z)$ term. For the higher spin-1 current, the two arbitrary coefficient tensors are inserted. We also insert the $(N, k)$ dependences in (3.3) from $N=3$ result of (3.2).

The primary condition of the higher spin- 1 current under the stress energy tensor (2.14) leads to the following OPE

$$
\left.T^{(1)}(z) \hat{T}(w)\right|_{\frac{1}{(z-w)^{2}}}=T^{(1)}(w) .
$$

In other words, there are no other singular terms in the $\operatorname{OPE} T^{(1)}(z) \hat{T}(w)$ except (3.4). The third-order pole is identically zero. From the condition of (3.4), one has the following relations

$$
\begin{aligned}
-\frac{1}{2(N+2)} A_{a} f_{\bar{b} \bar{c}}^{a} & =d_{\bar{b} \bar{c}}^{0}, \\
A_{a} f_{c}^{a \bar{b}} f_{\bar{b} d}^{c}-\frac{k}{(N+2)} A_{a} f_{{ }_{\bar{b}} \bar{c}}^{a} f^{\bar{b} \bar{c}}{ }_{d} & =2(k+N+2) A_{d} .
\end{aligned}
$$

The regular conditions with the six spin- 1 currents imply that

$$
T^{(1)}(z) A^{ \pm i}(w)=+\cdots .
$$

From the first condition (with upper sign) of (3.6), one has the two relations (corresponding to the first-order pole and second-order pole)

$$
A_{a} f_{d}^{\bar{b} \bar{c}} h_{\bar{b} \bar{c}}^{i} f_{e}^{a d}=0, \quad A_{a} f^{\bar{b} \bar{c} a} h_{\bar{b} \bar{c}}^{i}=0 .
$$


From the second condition (with lower sign) of (3.6), the following relations for the firstorder and the second-order poles hold

$$
d_{\bar{a}}^{0} \bar{c} h_{\bar{c} \bar{b}}^{i}=d_{\bar{b}}^{0} \bar{c} h_{\bar{c} \bar{a}}^{i}, \quad d^{0 \bar{a} \bar{b}} h_{\bar{a} \bar{b}}^{i}=0 .
$$

One has the following condition

$$
\left.T^{(1)}(z) G^{\mu}(w)\right|_{\frac{1}{(z-w)^{2}}}=0 .
$$

From this relation (3.8), the following relations are satisfied

$$
A^{\bar{a}} h_{\bar{a} \bar{b}}^{\mu}=0, \quad\left(\text { or } \quad A_{\bar{a}}=0\right) .
$$

When $\mu=0$ in this equation, one obtains the relation inside of the bracket. Using this result, for the $\mu=i$, one has $A^{\bar{a}} h_{\bar{a} \bar{b}}^{i}=0$ automatically. One can obtain the following relation from (3.5) and (3.9) as follows:

$$
A_{a}=d_{\bar{b} \bar{c}}^{0} f_{a}^{\bar{b} \bar{c}}{ }_{a}
$$

One can read off the explicit form for the second rank tensor $d_{\bar{a} \bar{b}}^{0}$ in $\mathrm{SU}(5)$ from [40] (or (3.2)) and generalize to the $\mathrm{SU}(N+2)$ case. In $\mathrm{SU}(5)$ case, one has the following $12 \times 12$ matrix representation

$$
d_{\bar{a} \bar{b}}^{0}=\left(\begin{array}{cccc}
0 & 0 & 1 & 0 \\
0 & 0 & 0 & -1 \\
-1 & 0 & 0 & 0 \\
0 & 1 & 0 & 0
\end{array}\right),
$$

where each element is $3 \times 3$ matrix. See appendix B for the $N$-generalization of the tensor $d_{\bar{a} \bar{b}}^{0}$ and other related tensors.

Furthermore, the tensor $d_{\bar{a} \bar{b}}^{0}$ satisfies the following properties (for $N=3,5,7,9$ )

$$
d_{\bar{a} \bar{c}}^{0} d^{0 \bar{c}}{ }_{\bar{b}}=g_{\bar{a} \bar{b}}, \quad d_{\bar{a} \bar{b}}^{0} f_{\bar{c} d}^{\bar{b}}=d_{\bar{c} \bar{b}}^{0} f_{\bar{a} d}^{\bar{b}} .
$$

The first equation of (3.12) is similar to the second equation of (2.9) while the second equation of (3.12) looks similar to the equation (3.11) of [29]. These will be used on (3.17) later.

Summarizing this subsection, one can rewrite $T^{(1)}(z)$ using (3.10) where the coefficient tensor appearing in the linear spin- 1 current can be written in terms of those appearing in the quadratic fermionic term,

$$
T^{(1)}(z)=-\frac{1}{2(k+N+2)} d_{\bar{a} \bar{b}}^{0} f^{\bar{a} \bar{b}}{ }_{c} V^{c}(z)+\frac{k}{2(k+N+2)^{2}} d_{\bar{a} \bar{b}}^{0} Q^{\bar{a}} Q^{\bar{b}}(z) .
$$

In the first term, there is sum over the index $c$ which can be expanded as the subgroup index and the coset index. This coset index term vanishes. Note that the structure constant satisfies $f_{\bar{c}}^{\bar{a} \bar{b}}=0$. This is true for the symmetric space. For $N=3$ case, the expression (3.2) does not contain the fields $V^{a}(z)$ where $a=1, \cdots, 6$ (or its complex conjugated ones). 
Compared to the previous spin- 1 currents of large $\mathcal{N}=4$ nonlinear superconformal algebra, the expression in (3.13) contains the $d_{\bar{a} \bar{b}}^{0}$ tensor. Because this higher spin-1 current is a singlet $(\mathbf{1}, \mathbf{1})$ under the $\mathrm{SU}(2)_{\hat{k}^{+}} \times \mathrm{SU}(2)_{\hat{k}^{-}}$, there is no any index corresponding to these groups. In the construction of the remaining higher spin currents, the presence of this $d_{\bar{a} \bar{b}}^{0}$ tensor occurs in all the OPEs in this paper.

\subsection{Four higher spin- $\frac{3}{2}$ currents}

Let us describe the next higher spin currents. Because the four spin- $\frac{3}{2}$ currents (2.7) and the higher spin-1 current (3.13) are given, one can calculate the following OPE

$$
G^{\mu}(z) T^{(1)}(w)=\frac{1}{(z-w)} G^{\prime \mu}(w)+\cdots
$$

The algebraic structure of (3.14) was already given in $N=3$ case. In other words, the higher spin- 1 current generates four higher spin- $\frac{3}{2}$ currents. One can calculate $G^{\prime \mu}(w)$ using the condition (3.7) and it turns out that

$$
G^{\prime \mu}(z)=\frac{i}{(k+N+2)} d_{\bar{a} \bar{b}}^{\mu} Q^{\bar{a}} V^{\bar{b}}(z)
$$

where the product of $d_{\bar{a}}^{0 \bar{c}}$ and $h_{\bar{c} \bar{b}}^{\mu}$ is defined via

$$
d_{\bar{a} \bar{b}}^{\mu} \equiv d_{\bar{a}}^{0 \bar{c}} h_{\bar{c} \bar{b}}^{\mu} .
$$

The $d_{\bar{a} \bar{b}}^{i}(i=1,2,3)$ are symmetric and traceless from the condition (3.7). Also the $\mu=0$ case in (3.16) leads to the $d_{\bar{a} \bar{b}}^{0}$ tensor. Compared to the previous spin- $\frac{3}{2}$ currents (2.5), the only difference is the presence of the second rank tensor in (3.16). The normalization and the field contents are the same. It is obvious that the higher spin- $\frac{3}{2}$ currents transform as $(\mathbf{2}, \mathbf{2})$ under the $\mathrm{SU}(2) \times \mathrm{SU}(2)$ as done in the four spin- $\frac{3}{2}$ currents in (2.5). They share the common $\mu$ indices.

One can calculate the OPE $G^{\prime \mu}(z) G^{\prime \nu}(w)$ using the relation (3.12) and the result is given by the opposite sign of (2.6)

$$
G^{\prime \mu}(z) G^{\prime \nu}(w)=-G^{\mu}(z) G^{\nu}(w)
$$

This is also observed in the context of asymptotic symmetry algebra in [49]. In other words, their singular terms have the above relation (3.17).

From (2.16) on the spin- $\frac{3}{2}$ currents, one can also have similar relations for the higher spin- $\frac{3}{2}$ currents as

$$
\begin{array}{ll}
\hat{G}_{11}^{\prime}(z)=\frac{1}{\sqrt{2}}\left(G^{\prime 1}-i G^{\prime 2}\right)(z), & \hat{G}_{12}^{\prime}(z)=-\frac{1}{\sqrt{2}}\left(G^{\prime 3}-i G^{\prime 0}\right)(z), \\
\hat{G}_{22}^{\prime}(z)=\frac{1}{\sqrt{2}}\left(G^{\prime 1}+i G^{\prime 2}\right)(z), & \hat{G}_{21}^{\prime}(z)=-\frac{1}{\sqrt{2}}\left(G^{\prime 3}+i G^{\prime 0}\right)(z) .
\end{array}
$$

Then by using the defining equation, one obtains the following OPE in the bispinor notation

$$
\hat{G}_{m n}(z) T^{(1)}(w)=\frac{1}{(z-w)} \hat{G}_{m n}^{\prime}(w)+\cdots, \quad(m, n=1,2) .
$$


Note that the currents $\hat{G}_{m n}^{\prime}(w)$ is denoted by $V_{\frac{1}{2}}^{(1) \alpha \beta}(w)$ in [20]. The higher spin- $\frac{3}{2}$ current $T_{+}^{\left(\frac{3}{2}\right)}(w)$ was appeared in the following OPE [40] (see also appendix $\mathrm{G}$ )

$$
\hat{G}_{21}(z) T^{(1)}(w)=\frac{1}{(z-w)}\left[\hat{G}_{21}+2 T_{+}^{\left(\frac{3}{2}\right)}\right](w)+\cdots .
$$

Then one can rewrite the first-order pole in (3.20) as the one in (3.19) and one has the following simple relation

$$
\begin{aligned}
T_{+}^{\left(\frac{3}{2}\right)}(z) & =\frac{1}{2}\left(\hat{G}_{21}^{\prime}-\hat{G}_{21}\right)(z) \\
& =-\frac{i}{2 \sqrt{2}(k+N+2)}\left(d^{3}+i d^{0}-h^{3}-i h^{0}\right)_{\bar{a} \bar{b}} Q^{\bar{a}} V^{\bar{b}}(z)
\end{aligned}
$$

Similarly, for completeness with the help of appendix G, one writes the other three higher spin- $\frac{3}{2}$ currents as follows:

$$
\begin{aligned}
T_{-}^{\left(\frac{3}{2}\right)}(z) & =\frac{1}{2}\left(\hat{G}_{12}^{\prime}+\hat{G}_{12}\right)(z)=-\frac{i}{2 \sqrt{2}(k+N+2)}\left(d^{3}-i d^{0}+h^{3}-i h^{0}\right)_{\bar{a} \bar{b}} Q^{\bar{a}} V^{\bar{b}}(z) \\
U^{\left(\frac{3}{2}\right)}(z) & =\frac{1}{2}\left(\hat{G}_{11}^{\prime}-\hat{G}_{11}\right)(z)=\frac{i}{2 \sqrt{2}(k+N+2)}\left(d^{1}-i d^{2}-h^{1}+i h^{2}\right)_{\bar{a} \bar{b}} Q^{\bar{a}} V^{\bar{b}}(z) \\
V^{\left(\frac{3}{2}\right)}(z) & =\frac{1}{2}\left(\hat{G}_{22}^{\prime}+\hat{G}_{22}\right)(z) \\
& =\frac{i}{2 \sqrt{2}(k+N+2)}\left(d^{1}+i d^{2}+h^{1}+i h^{2}\right)_{\bar{a} \bar{b}} Q^{\bar{a}} V^{\bar{b}}(z) .
\end{aligned}
$$

Summarizing this subsection, the expression in (3.15) contains the $d_{\bar{a} \bar{b}}^{\mu}$ tensor while the previous spin- $\frac{3}{2}$ currents of large $\mathcal{N}=4$ nonlinear superconformal algebra has the $h_{\bar{a} \bar{b}}^{\mu}$ tensor. Or $\hat{G}_{m n}^{2}(z)$ and $\hat{G}_{m n}^{\prime}(z)$ in $(3.19)$ are in the representation $(\mathbf{2}, \mathbf{2})$ under the $\mathrm{SU}(2)_{\hat{k}^{+}} \times \mathrm{SU}(2)_{\hat{k}^{-}}$. According to the defining OPE in (3.14), the role of $h_{\bar{a} \bar{b}}^{\mu}$ and $d_{\bar{a} \bar{b}}^{0}$ tensors was crucial. The former gives the $\mathrm{SO}(4)$ vector representation for the higher spin- $\frac{3}{2}$ currents and the latter gives the additional $\mu$-independence on them. ${ }^{10}$

${ }^{10}$ Note that for $N=3$ the above four higher spin- $\frac{3}{2}$ currents were written as

$$
\begin{aligned}
& T_{+}^{\left(\frac{3}{2}\right)}(z)=-\frac{\sqrt{2}}{(5+k)} \sum_{\left(\bar{A}, \bar{A}^{*}\right)=\left(\overline{4}, \bar{A}^{*}\right)}^{\left(\overline{6}, \overline{6}^{*}\right)} Q^{\bar{A}} V^{\bar{A}^{*}}(z), \quad T_{-}^{\left(\frac{3}{2}\right)}(z)=-\frac{\sqrt{2}}{(5+k)} \sum_{\left(\bar{A}, \bar{A}^{*}\right)=\left(\overline{4}, \overline{4}^{*}\right)}^{\left(\overline{6}, \overline{6}^{*}\right)} Q^{\bar{A}^{*}} V^{\bar{A}}(z), \\
& U^{\left(\frac{3}{2}\right)}(z)=-i \frac{\sqrt{2}}{(5+k)} \sum_{(\bar{A}, \bar{B})=(\overline{4}, \overline{1})}^{(\overline{\overline{6}}, \overline{3})} Q^{\bar{A}} V^{\bar{B}}(z), \quad V^{\left(\frac{3}{2}\right)}(z)=i \frac{\sqrt{2}}{(5+k)} \sum_{\left(\bar{A}^{*}, \bar{B}^{*}\right)=\left(\overline{4}^{*}, \overline{1}^{*}\right)}^{\left(\overline{6}^{*}, \overline{3}^{*}\right)} Q^{\bar{A}^{*}} V^{\bar{B}^{*}}(z) \text {. }
\end{aligned}
$$

From the representation in (B.2) and (B.4), the nonzero elements for $\left(d^{3}+i d^{0}-h^{3}-i h^{0}\right)_{\bar{a} \bar{b}}$ are given by $\overline{4} \overline{4}^{*}, \overline{5} \overline{5}^{*}$ and $\overline{6} \overline{6}^{*}$. Then one can check that $T_{+}^{\left(\frac{3}{2}\right)}(z)$ is consistent with the general expression in (3.21). One can also check other cases. 


\subsection{Six higher spin-2 currents}

One can generalize the equation (4.27) in [40] to the corresponding OPE for general $N$ (in fact $N=3,5,7,9)$ as follows:

$$
\begin{aligned}
\hat{G}_{11}(z) T_{-}^{\left(\frac{3}{2}\right)}(w)= & \frac{1}{(z-w)^{2}} \frac{2 N}{(N+k+2)}\left[i \hat{A}_{+}\right](w) \\
& +\frac{1}{(z-w)}\left[-U_{-}^{(2)}+\frac{1}{2} \partial\left\{\hat{G}_{11} T_{-}^{\left(\frac{3}{2}\right)}\right\}_{-2}\right](w)+\cdots
\end{aligned}
$$

From the relation (3.22), the left hand side of the OPE (3.23) can be written as

$$
\hat{G}_{11}(z) T_{-}^{\left(\frac{3}{2}\right)}(w)=\frac{1}{2} \hat{G}_{11}(z) \hat{G}_{12}^{\prime}(w)+\frac{1}{2} \hat{G}_{11}(z) \hat{G}_{12}(w) .
$$

Because the OPE $\hat{G}_{11}(z) \hat{G}_{12}(w)$ is already known for general $N$ (appendix D), one arrives at the following OPE

$$
\hat{G}_{11}(z) \hat{G}_{12}^{\prime}(w)=\frac{1}{(z-w)}\left[-2 U_{-}^{(2)}-\frac{4}{(N+k+2)} \hat{A}_{+} \hat{B}_{3}\right](w)+\cdots
$$

In other words, there are no second-order pole terms in (3.25) because they are cancelled each other in (3.24). From (3.25), one can write down the higher spin-2 current as follows:

$$
U_{-}^{(2)}(w)=-\frac{1}{2}\left\{\hat{G}_{11} \hat{G}_{12}^{\prime}\right\}_{-1}(w)-\frac{2}{(N+k+2)} \hat{A}_{+} \hat{B}_{3}(w)
$$

Let us emphasize that in (3.23) one should calculate the second-order pole to determine $U_{-}^{(2)}(w)$ for the relative $(N, k)$ dependent coefficient appearing in the descendant field of $\hat{A}_{+}(w)$ but in (3.26) one has to calculate the first-order pole only. Then it is straightforward to calculate the first term of (3.26) from (2.7), (2.16), (3.15) and (3.18) and it turns out (for $N=3,5,7$ ) that

$$
\begin{aligned}
U_{-}^{(2)}(w)= & \frac{1}{2(k+N+2)^{2}}\left[(k+N+2)\left(i d^{1}+d^{2}\right)_{\bar{a} \bar{b}} V^{\bar{a}} V^{\bar{b}}\right. \\
& \left.-\frac{1}{2}\left(h^{1}-i h^{2}\right)_{\bar{a} \bar{b}}\left(i d^{0}-d^{3}\right)_{\bar{c} \bar{d}} f^{\bar{b} \bar{d}} Q^{\bar{a}} Q^{\bar{c}} V^{e}\right](w)-\frac{2}{(N+k+2)}\left[\hat{A}_{+} \hat{B}_{3}\right](w) .
\end{aligned}
$$

Furthermore one can substitute (2.12) with (2.16) into the last term of (3.27)

$$
\begin{aligned}
U_{-}^{(2)}(w)= & \frac{1}{2(k+N+2)^{2}}\left[(k+N+2)\left(i d^{1}+d^{2}\right)_{\bar{a} \bar{b}} V^{\bar{a}} V^{\bar{b}}\right. \\
& \left.-\frac{1}{4 N} f_{e}^{\bar{c} \bar{d}}\left(h^{1}-i h^{2}\right)_{\bar{c} \bar{d}}\left(i d^{0}-h^{3}\right)_{\bar{a} \bar{b}} Q^{\bar{a}} Q^{\bar{b}} V^{e}\right](w),
\end{aligned}
$$


where the identities appearing in (E.3) are used. ${ }^{11}$ There are no quartic terms in (3.28) because the spin- $\frac{3}{2}$ current and the higher spin- $\frac{3}{2}$ current do not have any cubic terms.

Now one can describe the next higher spin-2 current. As in previous case, the equation (4.37) of [40] (or appendix G) implies the algebraic structure for the higher spin2 current $V_{+}^{(2)}(w)$. The OPE $\hat{G}_{22}(z) T_{+}^{\left(\frac{3}{2}\right)}(w)$ can be written in terms of $\hat{G}_{22}(z)$ with $\frac{1}{2}\left(\hat{G}_{21}^{\prime}-\hat{G}_{21}\right)(w)$ from (3.21). Then the second-order pole of (4.37) of [40] is canceled out the one of the OPE between $\hat{G}_{22}(z)$ and $\frac{1}{2} \hat{G}_{21}(w)$. By reading off the first-order pole in the OPE between $\hat{G}_{22}(z)$ and $\hat{G}_{21}^{\prime}(w)$, one obtains the following higher spin-2 current (for $N=3,5,7$ )

$$
\begin{aligned}
V_{+}^{(2)}(w)= & \frac{1}{2(k+N+2)^{2}}\left[(k+N+2)\left(-i d^{1}+d^{2}\right)_{\bar{a} \bar{b}} V^{\bar{a}} V^{\bar{b}}\right. \\
& \left.-\frac{1}{2}\left(i g+h^{3}\right)_{\bar{a} \bar{b}}\left(d^{1}+i d^{2}\right)_{\bar{c} \bar{d}} f^{\bar{b} \bar{d}} Q^{\bar{a}} Q^{\bar{c}} V^{e}\right](w)+\frac{2}{(N+k+2)}\left[\hat{A}_{-} \hat{B}_{3}\right](w) .
\end{aligned}
$$

Moreover, further substitution of (2.12) and (2.16) into the last term of (3.29) leads to the following simplified expression

$$
\begin{aligned}
V_{+}^{(2)}(w)= & \frac{1}{2(k+N+2)^{2}}\left[(k+N+2)\left(-i d^{1}+d^{2}\right)_{\bar{a} \bar{b}} V^{\bar{a}} V^{\bar{b}}\right. \\
& \left.+\frac{1}{4 N} f_{e}^{\bar{c} \bar{d}}\left(h^{1}+i h^{2}\right)_{\bar{c} \bar{d}}\left(i d^{0}-h^{3}\right)_{\bar{a} \bar{b}} Q^{\bar{a}} Q^{\bar{b}} V^{e}\right](w),
\end{aligned}
$$

where the identities appearing in (E.3) are used. Note that the complete expression of (3.30) looks similar to the one in (3.28). The only difference appears in the coefficients of $d_{\bar{a} \bar{b}}^{1}$ and $h_{\bar{c} \bar{d}}^{1}$. The U(1) charge introduced in [40] of (3.30) is opposite to the one of (3.28). ${ }^{12}$

Let us consider the two higher spin-2 currents with nonzero U(1) charges. The equation (4.23) of [40] (or appendix G) implies the algebraic structure for the higher spin2 current $U_{+}^{(2)}(w)$. The OPE $\hat{G}_{11}(z) T_{+}^{\left(\frac{3}{2}\right)}(w)$ can be written in terms of $\hat{G}_{11}(z)$ with $\frac{1}{2}\left(\hat{G}_{21}^{\prime}-\hat{G}_{21}\right)(w)$ from (3.21). Then the second-order pole of (4.23) of [40] is canceled

$$
\begin{aligned}
& { }^{11} \text { For } N=3 \text {, this current has the following form: } \\
& U_{-}^{(2)}(w)=-i \frac{2}{(5+k)^{2}} \sum_{\left(\bar{A}, \bar{A} \bar{A}^{*}\right)=\left(\overline{4}, \overline{A^{*}}\right)}^{\left(\overline{6}, \overline{6}^{*}\right)} Q^{\bar{A}} Q^{\bar{A}^{*}} V^{9}(w)-i \frac{2}{(5+k)} \sum_{(\bar{A}, \bar{B})=(\overline{1}, \overline{4})}^{(\overline{3}, \overline{6})} V^{\bar{A}} V^{\bar{B}}(w)-\frac{3 i}{(5+k)} \partial V^{9}(w) .
\end{aligned}
$$

One can see that the quadratic term can be checked by realizing that $\left(i d^{1}+d^{2}\right)_{\bar{a} \bar{b}}$ has nonzero term in the $\overline{1} \overline{4}, \overline{2} \overline{5}$ and $\overline{3} \overline{6}$ elements (and $\overline{4} \overline{1}, \overline{5} \overline{2}$ and $\overline{6} \overline{3}$ elements) from (B.2) and (B.4). The derivative term in the above can be written in terms of quadratic terms. The cubic terms can be checked from appendices (B.2) and (B.4) and the explicit values of nonzero structure constants in [40].

${ }^{12}$ For $N=3$, this current has the following form:

$$
V_{+}^{(2)}(w)=-i \frac{2}{(5+k)^{2}} \sum_{\left(\bar{A}, \bar{A}^{*}\right)=\left(\overline{4}, \overline{4}^{*}\right)}^{\left(\overline{6}, \overline{6}^{*}\right)} Q^{\bar{A}} Q^{\bar{A}^{*}} V^{9^{*}}(w)-i \frac{2}{(5+k)} \sum_{\left(\bar{A}^{*}, \bar{B}^{*}\right)=\left(\overline{1}^{*}, \overline{4}^{*}\right)}^{\left(\overline{3}^{*}, \overline{6}^{*}\right)} V^{\bar{A}^{*}} V^{\bar{B}^{*}}(w)+\frac{3 i}{(5+k)} \partial V^{9^{*}}(w) .
$$

The nonzero elements of $\left(-i d^{1}+d^{2}\right)_{\bar{a} \bar{b}}$ in (B.2) and (B.4) are given by in the $\overline{1}^{*} \overline{4}^{*}, \overline{2}^{*} \overline{5}^{*}$ and $\overline{3}^{*} \overline{6}^{*}$ (and $\overline{4}^{*} \overline{1}^{*}$, $\overline{5}^{*}, \overline{2}^{*}$ and $\left.\overline{6}^{*}, \overline{3}^{*}\right)$ and one can check the quadratic terms are identified. The derivative term is associated the ordering in the quadratic terms. One can check the cubic terms by looking at the nonzero structure constants appearing in [40] and appendices (B.2) and (B.4). 
out the one of the OPE between $\hat{G}_{11}(z)$ and $\frac{1}{2} \hat{G}_{21}(w)$. By reading off the first-order pole in the OPE between $\hat{G}_{11}(z)$ and $\hat{G}_{21}^{\prime}(w)$, one obtains the following higher spin-2 current (for $N=3,5,7$ )

$$
\begin{aligned}
U_{+}^{(2)}(w)= & -\frac{1}{2(k+N+2)^{2}}\left[k\left(i d^{1}+d^{2}\right)_{\bar{a} \bar{b}} Q^{\bar{a}} \partial Q^{\bar{b}}\right. \\
& \left.-\frac{1}{2}\left(h^{1}-i h^{2}\right)_{\bar{a} \bar{b}}\left(i d^{0}+d^{3}\right)_{\bar{c} \bar{d}} f^{\bar{b} \bar{d}} Q_{e}^{\bar{a}} Q^{\bar{c}} V^{e}\right](w)-\frac{2}{(N+k+2)}\left[\hat{A}_{3} \hat{B}_{-}\right](w) .
\end{aligned}
$$

The last term of (3.31) can be replaced with the expressions in section 2 .

The equation (4.41) of [40] (or appendix G) implies the algebraic structure for the higher spin-2 current $V_{-}^{(2)}(w)$. The OPE $\hat{G}_{22}(z) T_{-}^{\left(\frac{3}{2}\right)}(w)$ can be written in terms of $\hat{G}_{22}(z)$ with $\frac{1}{2}\left(\hat{G}_{12}^{\prime}+\hat{G}_{12}\right)(w)$ from (3.22). Then the second-order pole of (4.47) of [40] is canceled out the one of the OPE between $\hat{G}_{22}(z)$ and $\frac{1}{2} \hat{G}_{12}(w)$. By reading off the first-order pole in the OPE between $\hat{G}_{22}(z)$ and $\hat{G}_{12}^{\prime}(w)$, one obtains the following higher spin-2 current (for $N=3,5,7$ )

$$
\begin{aligned}
& V_{-}^{(2)}(w)=\frac{1}{2(k+N+2)^{2}}\left[k\left(i d^{1}-d^{2}\right)_{\bar{a} \bar{b}} Q^{\bar{a}} \partial Q^{\bar{b}}+\frac{1}{2}\left(i g-h^{3}\right)_{\bar{a} \bar{b}}\left(d^{1}+i d^{2}\right)_{\bar{c} \bar{d}} f^{\bar{b} \bar{d}} Q^{\bar{a}} Q^{\bar{c}} V^{e}\right](w) \\
& +\frac{2}{(N+k+2)}\left[\hat{A}_{3} \hat{B}_{+}\right](w) \text {. }
\end{aligned}
$$

One can also replace the last term of (3.32) with the expressions in section 2 .

The equation (4.16) of [40] (or appendix G) implies the algebraic structure for the higher spin-2 current $T^{(2)}(w)$. The OPE $\hat{G}_{21}(z) T_{-}^{\left(\frac{3}{2}\right)}(w)$ can be written in terms of $\hat{G}_{21}(z)$ with $\frac{1}{2}\left(\hat{G}_{12}^{\prime}+\hat{G}_{12}\right)(w)$ from (3.22). Then the third-order pole of (4.16) of [40] is canceled out the one of the OPE between $\hat{G}_{21}(z)$ and $\frac{1}{2} \hat{G}_{12}(w)$. By reading off the first-order pole in the OPE between $\hat{G}_{21}(z)$ and $\hat{G}_{12}^{\prime}(w)$, one obtains the following higher spin-2 current (for $N=3,5,7$ )

$$
\begin{aligned}
T^{(2)}(w)= & -\frac{1}{2(k+N+2)^{2}}\left[(k+N+2)\left(d^{0}-i d^{3}\right)_{\bar{a} \bar{b}} V^{\bar{a}} V^{\bar{b}}+k\left(d^{0}-i d^{3}\right)_{\bar{a} \bar{b}} Q^{\bar{a}} \partial Q^{\bar{b}}\right. \\
& \left.-\frac{1}{2}\left(i g-h^{3}\right)_{\bar{a} \bar{b}}\left(i d^{0}+d^{3}\right)_{\bar{c} \bar{d}} f^{\bar{b} \bar{d}} Q_{e}^{\bar{a}} Q^{\bar{c}} V^{e}\right](w) \\
& +\left[\frac{1}{2} \partial T^{(1)}+\frac{1}{(N+k+2)}\left(\hat{A}_{i} \hat{A}_{i}+\hat{B}_{i} \hat{B}_{i}-2 \hat{A}_{3} \hat{B}_{3}\right)+\frac{(k+N)}{(k+N+2 k N)} \hat{T}\right](w) .
\end{aligned}
$$

The last line of (3.33) comes from appendix G.

The equation (4.48) of [40] (or appendix G) implies the algebraic structure for the higher spin-2 current $W^{(2)}(w)$. The OPE $\hat{G}_{22}(z) U^{\left(\frac{3}{2}\right)}(w)$ can be written in terms of $\hat{G}_{22}(z)$ with $\frac{1}{2}\left(\hat{G}_{11}^{\prime}-\hat{G}_{11}\right)(w)$ from (3.22). Then the third-order pole of (4.48) of [40] is canceled out the one of the OPE between $\hat{G}_{22}(z)$ and $\frac{1}{2} \hat{G}_{11}(w)$. By reading off the first-order pole in the OPE between $\hat{G}_{22}(z)$ and $\hat{G}_{11}^{\prime}(w)$, one obtains the following higher spin-2 current 
(for $N=3,5,7)$

$$
\begin{aligned}
W^{(2)}(w)= & \frac{1}{2(k+N+2)^{2}}\left[(k+N+2)\left(d^{0}-i d^{3}\right)_{\bar{a} \bar{b}} V^{\bar{a}} V^{\bar{b}}+k\left(d^{0}+i d^{3}\right)_{\bar{a} \bar{b}} Q^{\bar{a}} \partial Q^{\bar{b}}\right. \\
& \left.+\frac{1}{2}\left(h^{1}-i h^{2}\right)_{\bar{a} \bar{b}}\left(d^{1}+i d^{2}\right)_{\bar{c} \bar{d}} f_{e}^{\bar{b} \bar{d}} Q^{\bar{a}} Q^{\bar{c}} V^{e}\right](w) \\
& +\left[-\frac{1}{2} \partial T^{(1)}+\frac{1}{(N+k+2)}\left(\hat{A}_{i} \hat{A}_{i}+\hat{B}_{i} \hat{B}_{i}+2 \hat{A}_{3} \hat{B}_{3}\right)+\hat{T}\right](w) .
\end{aligned}
$$

In this case, the last line of (3.34) comes from appendix G. ${ }^{13}$

It is useful to write the above higher spin-2 currents in terms of the $V_{1}^{(s) \pm i}(s=1, i=$ $1,2,3)$ introduced in [20]. Their equation (2.10) implies the following conditions

$$
\hat{T}(z) V_{1}^{(1) \pm i}(w)=\frac{1}{(z-w)^{2}} 2 V_{1}^{(1) \pm i}(w)+\frac{1}{(z-w)} \partial V_{1}^{(1) \pm i}(w)+\cdots .
$$

The regular conditions in (2.11) of [20] can be written as

$$
\left(\begin{array}{c}
\hat{A}_{i} \\
\hat{B}_{i}
\end{array}\right)(z) V_{1}^{(1) \mp j}(w)=+\cdots
$$

This implies that there are no singular terms between the triplet of spin-1 current $\hat{A}_{i}(z)\left[\hat{B}_{i}(z)\right]$ in the first [second] $\mathrm{SU}(2)$ factor and the triplet of the higher spin-2 current $V_{1}^{(1)-j}(w)\left[V_{1}^{(1)+j}(w)\right]$ in the second [first] $\mathrm{SU}(2)$ factor. Furthermore, one has the following OPEs

$$
\left(\begin{array}{c}
\hat{A}_{i} \\
\hat{B}_{i}
\end{array}\right)(z) V_{1}^{(1) \pm j}(w)=\frac{1}{(z-w)^{2}} a \delta_{i j} T^{(1)}(w)+\frac{1}{(z-w)} \epsilon^{i j k} V_{1}^{(1) \pm k}(w)+\cdots
$$

Therefore, the two triplets in the same SU(2) factor do have nontrivial OPEs.

By requiring the above conditions (3.36), (3.37) and (3.38), one obtains their three higher spin-2 currents as follows (for $N=3,5,7$ ):

$$
\begin{aligned}
& V_{1}^{(1)+1}(z)=\left[U_{-}^{(2)}-V_{+}^{(2)}+\frac{4}{(N+k+2)} \hat{A}_{1} \hat{B}_{3}\right](z) \\
& V_{1}^{(1)+2}(z)=\left[-i\left(U_{-}^{(2)}+V_{+}^{(2)}\right)+\frac{4}{(N+k+2)} \hat{A}_{2} \hat{B}_{3}\right](z) \\
& V_{1}^{(1)+3}(z)=\left[T^{(2)}-W^{(2)}+\frac{4}{(N+k+2)} \hat{A}_{3} \hat{B}_{3}+\frac{2 k N}{(N+k+2 k N)} \hat{T}\right](z) .
\end{aligned}
$$

\footnotetext{
${ }^{13}$ Strictly speaking, the higher spin-2 current $W^{(2)}(w)$ in [40] is not a primary current but a quasi-primary current. One can find the primary higher spin-2 current as follows (for $N=3,5,7,9$ ):

$$
\begin{aligned}
\tilde{W}^{(2)}(w) & \equiv W^{(2)}(w)-\frac{2 k N}{(k+N+2 k N)} \hat{T}(w) \\
& =\left[\frac{1}{2}\left\{\hat{G}_{11} \hat{G}_{22}^{\prime}\right\}_{-1}-\frac{1}{2} \partial T^{(1)}+\frac{2}{(N+k+2)}\left(\hat{A}_{i} \hat{A}_{i}+\hat{B}_{i} \hat{B}_{i}+2 \hat{A}_{3} \hat{B}_{3}\right)+\frac{(k+N)}{(k+N+2 k N)} \hat{T}\right](w) .
\end{aligned}
$$

Note that the coefficient of $\hat{T}(w)$ in $\tilde{W}^{(2)}(w)(3.35)$ is the same as the one of $\hat{T}(w)$ in $T^{(2)}(w)$. 
Note that the $\mathrm{U}(1)$ charge defined in [40] is not preserved in (3.39). It is not obvious to see the $\mathrm{SU}(2)$ index appearing in the left hand side of (3.39) in the right hand side explicitly. The other three higher spin-2 currents are given by (for $N=3,5,7$ )

$$
\begin{aligned}
& V_{1}^{(1)-1}(z)=\left[V_{-}^{(2)}-U_{+}^{(2)}-\frac{4}{(N+k+2)} \hat{A}_{3} \hat{B}_{1}\right](z) \\
& V_{1}^{(1)-2}(z)=\left[-i\left(V_{-}^{(2)}+U_{+}^{(2)}\right)-\frac{4}{(N+k+2)} \hat{A}_{3} \hat{B}_{2}\right](z), \\
& V_{1}^{(1)-3}(z)=\left[T^{(2)}+W^{(2)}-\frac{2}{(N+k+2)}\left(\hat{A}_{i} \hat{A}_{i}+\hat{B}_{i} \hat{B}_{i}\right)-\frac{2(N+k+k N)}{(N+k+2 k N)} \hat{T}\right](z) .
\end{aligned}
$$

Also in this case, the $\mathrm{U}(1)$ charge is not conserved. Because the right hand side of (3.39) and (3.40) are known from the previous results, one can simplify them further. ${ }^{14}$

Summarizing this subsection, the higher spin-2 currents are given by (3.28), (3.30), (3.31), (3.32), (3.33) and (3.34). They contain the quadratic and cubic terms in the Kac-Moody currents as well as the composite currents from the large $\mathcal{N}=4$ nonlinear superconformal algebra.

\subsection{Four higher spin- $\frac{5}{2}$ currents}

Now one can move the other higher spin currents. The (4.55) of [40] can be generalized to the following OPE

$$
\begin{aligned}
\hat{G}_{12}(z) W^{(2)}(w)= & \frac{1}{(z-w)^{2}}\left[\frac{(N+2 k+1)}{(N+k+2)} \hat{G}_{12}+\frac{(N-k)}{(N+k+2)} T_{-}^{\left(\frac{3}{2}\right)}\right](w) \\
& +\frac{1}{(z-w)}\left[W_{-}^{\left(\frac{5}{2}\right)}+\frac{1}{3} \partial\left\{\hat{G}_{12} W^{(2)}\right\}_{-2}\right](w)+\cdots .
\end{aligned}
$$

Then the spin- $\frac{5}{2}$ current (for $N=3,5,7$ ) can be read off

$$
W_{-}^{\left(\frac{5}{2}\right)}(w)=\left\{\hat{G}_{12} W^{(2)}\right\}_{-1}(w)-\frac{1}{3} \partial\left\{\hat{G}_{12} W^{(2)}\right\}_{-2}(w) .
$$

The second term can be obtained from the second-order pole of (3.43). From the explicit expressions of spin- $\frac{3}{2}$ current (2.7) with (2.16) and the higher spin-2 current (3.34), one

${ }^{14}$ That is,

$$
V_{1}^{(1)+j}(z)=\frac{i(-1)^{j+1}}{(k+N+2)^{2}}\left[(k+N+2) d_{\bar{a} \bar{b}}^{j} V^{\bar{a}} V^{\bar{b}}-\frac{1}{2} C_{\bar{a} \bar{b} \bar{c} \bar{d}}^{+j} f^{\bar{b} \bar{d}}{ }_{e} Q^{\bar{a}} Q^{\bar{c}} V^{e}\right](z),
$$

where the rank four tensor is introduced as follows:

$$
C_{\bar{a} \bar{b} \bar{c} \bar{d}}^{ \pm j} \equiv h_{\bar{a} \bar{b}}^{j} d_{\bar{c} \bar{d}}^{0} \pm \frac{1}{2} \epsilon^{j k l} h_{\bar{a} \bar{b} \bar{c}}^{k} d_{\bar{c} \bar{d}}^{l} .
$$

Similarly the right hand side of (3.40) can be further simplified as follows:

$$
V_{1}^{(1)-j}(z)=\frac{i}{(k+N+2)^{2}}\left[k d_{\bar{a} \bar{b}}^{j} Q^{\bar{a}} \partial Q^{\bar{b}}-\frac{1}{2} C_{\bar{a} \bar{b} \bar{c} \bar{d}}^{-j} f^{\bar{b} \bar{d}} Q_{e}^{\bar{a}} Q^{\bar{c}} V^{e}\right](z) .
$$

The constant $a$ in (3.38) is determined in next subsection and is fixed as $a=i$. After this simplification, one sees the $\mathrm{SU}(2) \times \mathrm{SU}(2)$ representations explicitly. 
obtains the following result

$$
\begin{aligned}
W_{-}^{\left(\frac{5}{2}\right)}(w)= & \frac{1}{\sqrt{2}(k+N+2)^{2}}\left[(k-1)\left(d^{0}+i d^{3}\right)_{\bar{a} \bar{b}} \partial Q^{\bar{a}} V^{\bar{b}}-(k+1)\left(d^{0}+i d^{3}\right)_{\bar{a} \bar{b}} Q^{\bar{a}} \partial V^{\bar{b}}\right. \\
& \left.+A_{\bar{a} \overline{d e}} Q^{\bar{a}} V^{\bar{d}} V^{e}+\frac{1}{4(k+N+2)} B_{\bar{a} \bar{b} \bar{c} \bar{d}} Q^{\bar{a}} Q^{\bar{b}} Q^{\bar{c}} V^{\bar{d}}\right](w)-\frac{1}{(N+k+2)}\left[\frac{4}{3} \partial \hat{G}_{12}\right. \\
& \left.+\frac{1}{3}(2 N+k+3) \partial \hat{G}_{12}^{\prime}-2 i\left(\hat{A}_{3}+\hat{B}_{3}\right) \hat{G}_{12}+i \hat{A}_{+} \hat{G}_{22}+i \hat{B}_{+} \hat{G}_{11}\right](w) .
\end{aligned}
$$

The second part (starting from $\partial \hat{G}_{12}(w)$ to the end) of (3.44) can be obtained by taking the OPEs between the spin- $\frac{3}{2}$ current $\hat{G}_{12}(z)$ and the last line of (3.34) (and the contributions from the derivative of the second order pole of (3.43)). All of these are easily determined from the basic OPEs in section 2 (and the OPE in (3.19)). Here we introduce the rank three tensor and the rank four tensor as follows:

$$
\begin{aligned}
A_{\bar{a} \bar{d} e} & \equiv\left[\left(-i g+h^{3}\right)_{\bar{a} \bar{b}} d_{\bar{c} \bar{d}}^{3}+\frac{1}{2}\left(d^{1}+i d^{2}\right)_{\bar{a} \bar{b}}\left(h^{1}-i h^{2}\right)_{\bar{c} \bar{d}}\right] f^{\bar{b} \bar{c}}, \\
B_{\bar{a} \bar{b} \bar{c} \bar{d}} & \equiv\left(-i h^{1}+h^{2}\right)_{\bar{a} \bar{b}}\left(i d^{1}+d^{2}\right)_{\bar{c} \bar{d}}+2 d_{\bar{a} \bar{b}}^{0}\left(g+i h^{3}\right)_{\bar{c} \bar{d}} .
\end{aligned}
$$

In the rank three tensor, there is a $\mathrm{SU}(N+2)$ index. In the rank four tensor, all the indices are living in the Wolf space coset. They will appear in the construction of the higher spin-3 current later.

In particular, the quartic term in the second line of (3.44) can be analyzed as follows. Let us focus on the OPE $\hat{G}_{12}(z)$ and the third term of the higher spin-2 current in (3.34). Then according to the last equation of appendix (F.1), one has the quartic term in the right hand side of (F.1). By multiplying the correct coefficients, one arrives at the final expression in (3.44). Note that in (3.44) there is no quintic fermionic term because the spin- $\frac{3}{2}$ current has no cubic term in the Wolf space coset. For the nonsymmetric space, one expects that there should be such quintic term.

Similarly, from appendix G (the first equation of (G.4)), one can read off the next higher spin- $\frac{5}{2}$ current and it turns out, from the spin- $\frac{3}{2}$ current (2.7) with (2.16) and the higher spin-2 current (3.27), that (for $N=3,5,7$ )

$$
\begin{aligned}
U^{\left(\frac{5}{2}\right)}(w)= & \left\{\hat{G}_{21} U_{-}^{(2)}\right\}_{-1}(w)-\frac{1}{3} \partial\left\{\hat{G}_{21} U_{-}^{(2)}\right\}_{-2}(w) \\
= & \frac{i}{\sqrt{2}(k+N+2)^{2}}\left[(2 k+N)\left(d^{1}-i d^{2}\right)_{\bar{a} \bar{b}} \partial Q^{\bar{a}} V^{\bar{b}}+N\left(d^{1}-i d^{2}\right)_{\bar{a} \bar{b}} Q^{\bar{a}} \partial V^{\bar{b}}\right. \\
& \left.+A_{\bar{a} \bar{d} e}^{1} Q^{\bar{a}} V^{\bar{d}} V^{e}+\frac{1}{2(k+N+2)} d_{\bar{a} \bar{b}}^{0}\left(h^{1}-i h^{2}\right)_{\bar{c} \bar{d}} Q^{\bar{a}} Q^{\bar{b}} Q^{\bar{c}} V^{\bar{d}}\right](w) \\
& +\frac{1}{(N+k+2)}\left[\frac{1}{3} \partial \hat{G}_{11}-\frac{1}{3}(N+2 k+1) \partial \hat{G}_{11}^{\prime}-2 i \hat{G}_{11} \hat{B}_{3}+i \hat{A}_{+} \hat{G}_{21}\right](w),
\end{aligned}
$$

where the tensor is given by

$$
A_{\bar{a} \bar{d} e}^{1} \equiv\left[\left(i g+h^{3}\right)_{\bar{a} \bar{b}}\left(i d^{1}+d^{2}\right)_{\bar{c} \bar{d}}+\frac{1}{2}\left(h^{1}-i h^{2}\right)_{\bar{a} \bar{b}}\left(d^{0}-i d^{3}\right)_{\bar{c} \bar{d}}\right] f_{e^{b}}^{\bar{b} \bar{c}}
$$


As described before, the last line of (3.46) can be obtained from the OPE between $\hat{G}_{21}(z)$ and the derivative of the second order pole in the first equation in (G.4). The quartic term in the second line (in the final expression) of (3.46) can be analyzed as follows. The OPE $\hat{G}_{21}(z)$ and the second term of the higher spin-2 current in (3.27) implies the last equation of appendix (F.1). One has the quartic term in the right hand side of (F.1). By multiplying the correct coefficients, one arrives at the final expression in (3.46). Note that in (3.46) there is no quintic fermionic term as described before.

One can continue to describe the following higher spin- $\frac{5}{2}$ current with the help of the spin- $\frac{3}{2}$ current (2.7) with (2.16) and the higher spin-2 current (3.32) and it turns out that (for $N=3,5,7$ )

$$
\begin{aligned}
V^{\left(\frac{5}{2}\right)}(w)= & \left\{\hat{G}_{21} V_{-}^{(2)}\right\}_{-1}(w)-\frac{1}{3} \partial\left\{\hat{G}_{21} V_{-}^{(2)}\right\}_{-2}(w) \\
= & \frac{i}{\sqrt{2}(k+N+2)^{2}}\left[k\left(d^{1}+i d^{2}\right)_{\bar{a} \bar{b}} Q^{\bar{a}} \partial V^{\bar{b}}-k\left(d^{1}+i d^{2}\right)_{\bar{a} \bar{b}} \partial Q^{\bar{a}} V^{\bar{b}}\right. \\
& \left.+A_{\bar{b} \bar{c} e}^{2} Q^{\bar{b}} V^{\bar{c}} V^{e}+\frac{1}{4(N+k+2)} B_{\bar{a} \bar{b} \bar{c} \bar{d}}^{2} Q^{\bar{a}} Q^{\bar{b}} Q^{\bar{c}} V^{\bar{d}}\right](w) \\
& -\frac{1}{(N+k+2)}\left[\frac{1}{3} \partial \hat{G}_{22}+\frac{1}{3}(2 N+k+1) \partial \hat{G}_{22}^{\prime}-2 i \hat{A}_{3} \hat{G}_{22}+i \hat{G}_{21} \hat{B}_{+}\right](w),
\end{aligned}
$$

where the two tensors are

$$
\begin{aligned}
A_{\bar{b} \bar{c} e}^{2} & \equiv\left(g-i h^{3}\right)_{\bar{a}[\bar{b}}\left(d^{1}+i d^{2}\right)_{\bar{c}] \bar{d}} f_{e}^{\bar{a} \bar{d}} \\
B_{\bar{a} \bar{b} \bar{c} \bar{d}}^{2} & \equiv\left(h^{1}+i h^{2}\right)_{\bar{a} \bar{b}}\left(d^{0}-i d^{3}\right)_{\bar{c} \bar{d}}+2 i h_{\bar{a} \bar{b}}^{3}\left(d^{1}+i d^{2}\right)_{\bar{c} \bar{d}}-2 d_{\bar{a} \bar{b}}^{0}\left(h^{1}+i h^{2}\right)_{\bar{c} \bar{d}} .
\end{aligned}
$$

The second equation of appendix (G.4) is used. The derivative of the second order pole in the second equation of (G.4) with the spin- $\frac{3}{2}$ current gives the last line of (3.47). Also the cubic and quartic terms in the second line (in the final expression) of (3.47) can be obtained using the property of appendix (F.1).

Finally the last higher spin- $\frac{5}{2}$-current can be obtained similarly. From the expressions of the spin- $\frac{3}{2}$ current (2.7) with (2.16) and the higher spin-2 current (3.34), one arrives at the following result (from the third equation of appendix (G.4)) (for $N=3,5,7$ )

$$
\begin{aligned}
W_{+}^{\left(\frac{5}{2}\right)}(w)= & \left\{\hat{G}_{21} W^{(2)}\right\}_{-1}(w)-\frac{1}{3} \partial\left\{\hat{G}_{21} W^{(2)}\right\}_{-2}(w) \\
= & \frac{1}{\sqrt{2}(k+N+2)^{2}}\left[(N+2 k+1)\left(d^{0}-i d^{3}\right)_{\bar{a} \bar{b}} \partial Q^{\bar{a}} V^{\bar{b}}+(N+1)\left(d^{0}-i d^{3}\right)_{\bar{a} \bar{b}} Q^{\bar{a}} \partial V^{\bar{b}}\right. \\
& \left.+A_{\bar{a} \bar{d} e}^{3} Q^{\bar{a}} V^{\bar{d}} V^{e}+\frac{1}{4(k+N+2)} B_{\bar{a} \bar{b} \bar{c} \bar{d}}^{3} Q^{\bar{a}} Q^{\bar{b}} Q^{\bar{c}} V^{\bar{d}}\right](w)-\frac{1}{(N+k+2)}\left[\frac{4}{3} \partial \hat{G}_{21}\right. \\
& \left.+\frac{1}{3}(N+2 k+3) \partial \hat{G}_{21}^{\prime}+2 i\left(\hat{A}_{3}+\hat{B}_{3}\right) \hat{G}_{21}-i \hat{A}_{-} \hat{G}_{11}-i \hat{B}_{-} \hat{G}_{22}\right](w),
\end{aligned}
$$

where we have the following tensors

$$
\begin{aligned}
A_{\bar{a} \bar{d} e}^{3} & \equiv\left[\left(i g+h^{3}\right)_{\bar{a} \bar{b}} d_{\bar{c} \bar{d}}^{3}+\frac{1}{2}\left(h^{1}-i h^{2}\right)_{\bar{a} \bar{b}}\left(d^{1}+i d^{2}\right)_{\bar{c} \bar{d}}\right] f^{\bar{b} \bar{c}}{ }_{e}, \\
B_{\bar{a} \bar{b} \bar{c} \bar{d}}^{3} & \equiv\left(h^{1}-i h^{2}\right)_{\bar{a} \bar{b}}\left(d^{1}+i d^{2}\right)_{\bar{c} \bar{d}}+2 d_{\bar{a} \bar{b}}^{0}\left(g-i h^{3}\right)_{\bar{c} \bar{d}} .
\end{aligned}
$$


Summarizing this subsection, the higher spin- $\frac{5}{2}$ currents are given by (3.44), (3.46), (3.47) and (3.48). They contain the quadratic, cubic and quartic terms in the Kac-Moody currents as well as the composite currents from the large $\mathcal{N}=4$ nonlinear superconformal algebra. ${ }^{15}$

\subsection{One higher spin-3 current}

Let us describe the final higher spin-3 current. The equation (4.59) of [40] can be generalized to the following result

$$
\begin{aligned}
\hat{G}_{21}(z) W_{-}^{\left(\frac{5}{2}\right)}(w)= & \frac{1}{(z-w)^{3}}\left[a_{1} \hat{A}_{3}+a_{2} \hat{B}_{3}+a_{3} T^{(1)}\right](w) \\
& +\frac{1}{(z-w)^{2}}\left[a_{4} \hat{T}+a_{5} T^{(2)}+a_{6} W^{(2)}+a_{7}\left(\hat{A}_{1} \hat{A}_{1}+\hat{A}_{2} \hat{A}_{2}\right)+a_{8} \hat{A}_{3} \hat{A}_{3}\right. \\
& \left.+a_{9} \hat{A}_{3} \hat{B}_{3}+a_{10}\left(\hat{B}_{1} \hat{B}_{1}+\hat{B}_{2} \hat{B}_{2}\right)+a_{11} \hat{B}_{3} \hat{B}_{3}+a_{12}\left(T^{(1)} \hat{A}_{3}-T^{(1)} \hat{B}_{3}\right)\right](w) \\
& +\frac{1}{(z-w)}\left[\frac{1}{4} \partial\left\{\hat{G}_{21} W_{-}^{\left(\frac{5}{2}\right)}\right\}_{-2}+a_{13}\left(\hat{T} \hat{A}_{3}-\frac{1}{2} \partial^{2} \hat{A}_{3}\right)+a_{14}\left(\hat{T} \hat{B}_{3}-\frac{1}{2} \partial^{2} \hat{B}_{3}\right)\right. \\
& \left.+a_{15}\left(\hat{T} T^{(1)}-\frac{1}{2} \partial^{2} T^{(1)}\right)+W^{(3)}\right](w)+\cdots,
\end{aligned}
$$

where the coefficients are given in (H.1). Then the higher spin-3 current can be written as

$$
\begin{aligned}
W^{(3)}(w)= & \left\{\hat{G}_{21} W_{-}^{\left(\frac{5}{2}\right)}\right\}_{-1}(w)-\left[\frac{1}{4} \partial\left\{\hat{G}_{21} W_{-}^{\left(\frac{5}{2}\right)}\right\}_{-2}+a_{13}\left(\hat{T} \hat{A}_{3}-\frac{1}{2} \partial^{2} \hat{A}_{3}\right)\right. \\
& \left.+a_{14}\left(\hat{T} \hat{B}_{3}-\frac{1}{2} \partial^{2} \hat{B}_{3}\right)+a_{15}\left(\hat{T} T^{(1)}-\frac{1}{2} \partial^{2} T^{(1)}\right)\right](w) .
\end{aligned}
$$

$$
\begin{aligned}
& V_{\frac{3}{2}, 11}^{(1)}(z)=2 i U^{\left(\frac{5}{2}\right)}(z) \\
& +\frac{4}{(N+k+2)}\left[\hat{A}_{+} \hat{G}_{21}+\hat{A}_{+} T_{+}^{\left(\frac{3}{2}\right)}-\hat{B}_{-} \hat{G}_{12}+2 \hat{B}_{-} T_{-}^{\left(\frac{3}{2}\right)}+2 \hat{B}_{3} U^{\left(\frac{3}{2}\right)}-\frac{2 i}{3} \partial U^{\left(\frac{3}{2}\right)}\right](z), \\
& V_{\frac{3}{2}, 22}^{(1)}(z)=-2 i V^{\left(\frac{5}{2}\right)}(z) \\
& +\frac{4}{(N+k+2)}\left[\hat{A}_{-} \hat{G}_{12}-2 \hat{A}_{-} T_{-}^{\left(\frac{3}{2}\right)}-2 \hat{A}_{3} V^{\left(\frac{3}{2}\right)}-\hat{B}_{+} T_{+}^{\left(\frac{3}{2}\right)}+\frac{2 i}{3} \partial V^{\left(\frac{3}{2}\right)}\right](z), \\
& V_{\frac{3}{2}, 21}^{(1)}(z)=2 i W_{+}^{\left(\frac{5}{2}\right)}(z)+\frac{4}{(N+k+2)}\left[\hat{A}_{-} \hat{G}_{11}+\hat{A}_{-} U^{\left(\frac{3}{2}\right)}-2 \hat{A}_{3} \hat{G}_{21}-2 \hat{A}_{3} T_{+}^{\left(\frac{3}{2}\right)}\right. \\
& \left.+\hat{B}_{-} \hat{G}_{22}-\hat{B}_{-} V^{\left(\frac{3}{2}\right)}+2 \hat{B}_{3} T_{+}^{\left(\frac{3}{2}\right)}+\frac{2 i}{3} \partial \hat{G}_{21}\right](z), \\
& V_{\frac{3}{2}, 12}^{(1)}(z)=-2 i W_{-}^{\left(\frac{5}{2}\right)}(z)+\frac{4}{(N+k+2)}\left[\hat{A}_{+} \hat{G}_{22}-\hat{A}_{+} V^{\left(\frac{3}{2}\right)}-2 \hat{A}_{3} \hat{G}_{12}+2 \hat{A}_{3} T_{-}^{\left(\frac{3}{2}\right)}\right. \\
& \left.+\hat{B}_{+} \hat{G}_{11}+\hat{B}_{+} U^{\left(\frac{3}{2}\right)}-2 \hat{B}_{3} T_{-}^{\left(\frac{3}{2}\right)}-\frac{2 i}{3} \partial \hat{G}_{12}\right](z) .
\end{aligned}
$$

Note that in this calculation, the constant $a$ appeared in the description of (3.41) and (3.42) is determined by $a=i$. It is not obvious to reexpress these in $\mathrm{SU}(2) \times \mathrm{SU}(2)$ manifest way as done in the higher spin- 2 currents. It would be interesting to obtain these higher spin- $\frac{5}{2}$ currents with manifest $(\mathbf{2}, \mathbf{2})$ representation of $\mathrm{SU}(2) \times \mathrm{SU}(2)$. 
Note that in order to obtain the second term of (3.50), one should differentiate the second order pole in (3.49). After calculating the first-order pole in the OPE between $\hat{G}_{21}(z)(2.7)$ with (2.16) and $W_{-}^{\left(\frac{5}{2}\right)}(w)(3.44)$, the first term of (3.50) is given by (for $N=3,5$ )

$$
\begin{aligned}
\left\{\hat{G}_{21} W_{-}^{\left(\frac{5}{2}\right)}\right\}_{-1}(w)= & \frac{i}{2(k+N+2)^{3}} \\
& \times\left[2 i(k+N+2) A_{\bar{a} \bar{d} e} V^{\bar{a}} V^{\bar{d}} V^{e}-4(k+N+2)\left(i k d^{0}-d^{3}\right)_{\bar{a} \bar{b}} V^{\bar{a}} \partial V^{\bar{b}}\right. \\
& -i(k-1)(k+N+2) d_{\bar{a} \bar{b}}^{0} f^{\bar{a} \bar{b}}{ }_{c} \partial^{2} V^{c}+\left((k+1) C_{\bar{c} \bar{a} e}+F_{\bar{a} \bar{c} e}\right) Q^{\bar{a}} Q^{\bar{c}} \partial V^{e} \\
& +\left((k-1) C_{\bar{a} \bar{c} e}+(k+1) C_{\bar{c} \bar{a} e}+k D_{\bar{a} \bar{c} e}+F_{\bar{a} \bar{c} e}\right) Q^{\bar{a}} \partial Q^{\bar{c}} V^{e} \\
& +E_{\bar{a} \bar{c} e f} Q^{\bar{a}} Q^{\bar{c}} V^{e} V^{f}+\left(G+\frac{3}{4} I\right)_{\bar{a} \bar{c} \bar{d} \bar{f}} Q^{\bar{a}} Q^{\bar{c}} V^{\bar{d}} V^{\bar{f}}+2 i k(k-1) d_{\bar{a} \bar{b}}^{0} \partial Q^{\bar{a}} \partial Q^{\bar{b}} \\
& +\frac{1}{4(k+N+2)} J_{\bar{a} \bar{b} \bar{c} \bar{d} h} Q^{\bar{a}} Q^{\bar{b}} Q^{\bar{c}} Q^{\bar{d}} V^{h}+\frac{k}{4(k+N+2)} K_{\bar{a} \bar{b} \bar{c} \bar{d}} Q^{\bar{a}} Q^{\bar{b}} Q^{\bar{c}} \partial Q^{\bar{d}} \\
& \left.+k\left(-2(k+1)\left(i d^{0}-d^{3}\right)+\frac{1}{2} H\right)_{\bar{a} \bar{c}} Q^{\bar{a}} \partial^{2} Q^{\bar{c}}\right](w) \\
& -\frac{1}{(N+k+2)}\left[\frac{4}{3} \partial\left\{\hat{G}_{21} \hat{G}_{12}\right\}_{-1}+\frac{(2 N+k+3)}{3} \partial\left\{\hat{G}_{21} \hat{G}_{12}^{\prime}\right\}_{-1}-2 \hat{G}_{21} \hat{G}_{12}\right. \\
& -\hat{G}_{11} \hat{G}_{22}-\hat{G}_{22} \hat{G}_{11}-2 i\left(\hat{A}_{3}+\hat{B}_{3}\right)\left\{\hat{G}_{21} \hat{G}_{12}\right\}_{-1}+i \hat{A}_{+}\left\{\hat{G}_{21} \hat{G}_{22}\right\}_{-1} \\
& \left.+i \hat{B}_{+}\left\{\hat{G}_{21} \hat{G}_{11}\right\}_{-1}\right](w),
\end{aligned}
$$

where the various tensors are

$$
\begin{aligned}
C_{\bar{a} \bar{c} e} & \equiv\left(i g+h^{3}\right)_{\bar{a} \bar{b}}\left(d^{0}+i d^{3}\right)_{\bar{c} \bar{d}} f_{e}^{\bar{b} \bar{d}} \\
E_{\bar{a} \bar{c} e f} & \equiv A_{\bar{a} \bar{d} e} f_{f}^{\bar{d} b}\left(i g-h^{3}\right)_{\bar{b} \bar{c}} \\
F_{\bar{a} \bar{c} e} & \equiv E_{\bar{a} \bar{c} b d} f_{e}^{b d} \\
D_{\bar{a} \bar{c} e} & \equiv\left[\left(i g-h^{3}\right)_{\bar{a} \bar{b}}\left(d^{0}-i d^{3}\right)_{\bar{c} \bar{d}}-\left(d^{1}+i d^{2}\right)_{\bar{a} \bar{b}}\left(i h^{1}+h^{2}\right)_{\bar{c} \bar{d}}\right] f^{\bar{b} \bar{d}}, \\
G_{\bar{a} \bar{c} \bar{d} \bar{f}} & \equiv A_{\bar{a} \bar{d} \bar{e}}\left(i g-h^{3}\right)_{\bar{b} \bar{c}} f^{e \bar{b}} \overline{\bar{f}} \\
H_{\bar{a} \bar{c}} & \equiv A_{\bar{a} \bar{d} \bar{e}}\left(i g-h^{3}\right)_{\bar{b} \bar{c}} f^{e \bar{d} \bar{b}}=-4 N\left(i d^{0}-d^{3}\right)_{\bar{a} \bar{c} \bar{c}} \\
I_{\bar{a} \bar{c} \bar{f} \bar{f}} & \equiv\left(i g+h^{3}\right)^{\bar{e}}{ }_{\bar{d}} B_{[\bar{e} \bar{a} \bar{c}] \bar{f}} \\
J_{\bar{a} \bar{b} \bar{c} \bar{d} h} & \equiv B_{\bar{a} \bar{b} \bar{c} \bar{e}}\left(i g+h^{3}\right)_{\bar{d} \bar{f}} f_{h}^{\bar{e} \bar{f}} \\
K_{\bar{a} \bar{b} \bar{c} \bar{d}} & \equiv 2 i\left(h^{1}+i h^{2}\right)_{\bar{a} \bar{b}}\left(d^{1}-i d^{2}\right)_{\bar{c} \bar{d}}+4 d_{\bar{a} \bar{b}}^{0}\left(i g-h^{3}\right)_{\bar{c} \bar{d} .}
\end{aligned}
$$

The $A_{\bar{a} \bar{d} e}$ and $B_{\bar{a} \bar{b} \bar{c} \bar{e}}$ tensors are given in (3.45). The identity containing $H_{\bar{a} \bar{c}}$ is checked by low values of $N=3,5,7$.

One can analyze each term in (3.51). For example, the first term consists of the cubic term. Let us consider the OPE $\hat{G}_{21}(z) W_{-}^{\left(\frac{5}{2}\right)}(w)$ where the cubic term is taken from the higher spin- $\frac{5}{2}$ current. According to the third equation of appendix (H.2), there exists a first term in the right hand side. By combining the right coefficient from the spin- $\frac{3}{2}$ current and the higher spin- $\frac{5}{2}$ current, one checks that the final corresponding expression is given by the first term in (3.51). The last three lines in (3.51) come from the OPE between 
$\hat{G}_{21}(z)$ and the composite fields appearing in the second part of (3.44). In order to obtain these expressions completely, appendices D and G are needed.

Summarizing this subsection, one way to obtain the last higher spin-3 current is given (the OPEs $\hat{G}_{22}(z) U^{\left(\frac{5}{2}\right)}(w)$ or $\hat{G}_{11}(z) V^{\left(\frac{5}{2}\right)}(w)$ provide the higher spin-3 current). The highest order is given by the sextic term which appears in $\hat{T} \hat{B}_{3}(w)$ or $\hat{T} T^{(1)}(w)$. There exists a cubic term in the bosonic spin-1 currents as one expected.

Therefore, the 16 higher spin currents in (3.1) are obtained in this section. Because they are written in terms of Kac-Moody currents explicitly, one observes that their zero mode can be determined and this will be the open problem to accomplish further in the context of three-point function.

\section{Conclusions and outlook}

We have determined the 16 higher spin currents with spins $\left(1, \frac{3}{2}, \frac{3}{2}, 2\right),\left(\frac{3}{2}, 2,2, \frac{5}{2}\right)$, $\left(\frac{3}{2}, 2,2, \frac{5}{2}\right)$ and $\left(2, \frac{5}{2}, \frac{5}{2}, 3\right)$ in the $\mathcal{N}=4$ superconformal Wolf space coset $\frac{\mathrm{SU}(N+2)}{\mathrm{SU}(N) \times \mathrm{SU}(2) \times \mathrm{U}(1)}$ using the bosonic and fermionic Kac-Moody currents.

Let us describe the future directions as follows:

- Three-point functions

As a second step to the construction of three point function [50-54] for the two scalars and one higher spin current, one should calculate the eigenvalue equations for the zero modes of the higher spin currents. In [4], one of the simple representation of the minimal representations is given by $Q_{-\frac{1}{2}}^{\bar{A}} \mid 0>$ where $\bar{A}=1,2, \cdots, 2 N$ corresponding to $(0 ; f)$ representation where the first element is an integrable highest weight representation of $\mathrm{SU}(N+2)$ and the second element is the one of $\mathrm{SU}(N)$. The above state contains the group index for the fundamental representation of $\mathrm{SU}(N)$ with two dimensional SU(2) representation. Then the eigenvalue equation for the zero mode of spin- 2 current acting on the above state can be calculated. One can do this by considering the zero mode of (2.14) and performing it into the above state. Or one can obtain the OPE between the spin-2 stress energy tensor $L(z)$ and the spin- $\frac{1}{2}$ current $Q^{\bar{A}}(w)$ and read off the relevant commutator relation. According to the result of [4], the conformal dimension $h(0 ; f)$ is given by $h(0 ; f)=\frac{\left(k+\frac{3}{2}\right)}{2(N+k+2)}$ which is equal to the one for BPS bound. That is, one should have $L_{0} Q_{-\frac{1}{2}}^{\bar{A}}\left|0>=h(0 ; f) Q_{-\frac{1}{2}}^{\bar{A}}\right| 0>$. It would be interesting to see this behavior by analyzing the results of this paper carefully. Furthermore, there exist other 10 currents and 16 higher spin currents. It is an open problem to construct the zero mode eigenvalue equations and obtain the various three point functions with two scalars together with each (higher spin) current.

- An extension of large $\mathcal{N}=4$ linear superconformal algebra

It would be interesting to obtain the higher spin currents in the context of an extension of large $\mathcal{N}=4$ linear superconformal algebra by adding the four spin- $\frac{1}{2}$ currents and one spin-1 current. As described in section 2, the spin- $\frac{3}{2}$ currents have the cubic 
fermionic terms. This can be observed in the explicit transformations between the spin-2, spin- $\frac{3}{2}$ and spin- 1 currents in the large $\mathcal{N}=4$ nonlinear superconformal algebra and those in the large $\mathcal{N}=4$ linear superconformal algebra, initiated by Goddard and Schwimmer in [26]. Furthermore, from the work of [39], one expects that one can obtain the 16 currents with the various tensors in terms of the spin- 1 and spin- $\frac{1}{2}$ Kac-Moody currents in (2.1). Due to the presence of the extra $(4+1)$ currents, there are more rooms for the coset space $\frac{\mathrm{SU}(N+2)}{\mathrm{SU}(N)}$. Once the above construction for the large $\mathcal{N}=4$ linear superconformal algebra is done, then it is straightforward to determine the higher spin currents by following the works of $[40,41]$ and this paper. One of the motivations for this direction is to provide an extension of small $\mathcal{N}=4$ superconformal algebra (one spin- 2 current, four spin- $\frac{3}{2}$ currents and three spin- 1 currents) by taking the appropriate limits on the levels in the theory. In the end, one obtains the OPEs for the extension of small $\mathcal{N}=4$ superconformal algebra which is related to the type IIB string compactification of $A d S_{3} \times \mathbf{S}^{3} \times \mathbf{T}^{4}$. It is not clear whether the extension of small $\mathcal{N}=4$ superconformal algebra can be obtained from the extension of large $\mathcal{N}=4$ nonlinear superconformal algebra because there exist nonlinear terms in the spin- 1 currents of the OPE between the spin- $\frac{3}{2}$ currents. Any simple transformations do not decouple the three spin-1 currents in these nonlinear terms of the OPEs.

- The application to an orthogonal Wolf space

One can apply the results of this paper to other type of Wolf space coset. There exists an orthogonal Wolf space coset by changing the unitary group to the orthogonal group. The relevant works in the orthogonal group are given by [55-61]. At the level of large $\mathcal{N}=4$ nonlinear superconformal algebra, there are no much differences between the unitary Wolf space coset theory and the orthogonal Wolf space coset theory because the role of $\mathrm{SU}(2) \times \mathrm{SU}(2)$ group is replaced with $\mathrm{SO}(3) \times \mathrm{SO}(3)$ group. The main difference between them arises when one tries to determine the extension of large $\mathcal{N}=4$ nonlinear superconformal algebra because the lowest $\mathcal{N}=4$ higher spin multiplet contains the higher spin-2 current as its lowest component rather than higher spin-1 current. This higher spin-2 current behaves as a singlet under the above $\mathrm{SU}(2) \times \mathrm{SU}(2)$. From the results of this paper, it is useful to recall that the higher spin-2 currents, $T^{(2)}(z)$ and $W^{(2)}$, contain $d_{\bar{a} \bar{b}}^{\mu}$ tensor, $h_{\bar{a} \bar{b}}^{\mu}$ tensor and $f^{\bar{a} \bar{b}}{ }_{c}$ structure constant. In order to determine the tensorial structure in the higher spin-2 current, the low $N$ value results for the work of [62] will be very useful because they will give the explicit realizations on the above tensors. It is an open problem why the higher spin-1 current like the $T^{(1)}(z)$ (and other four higher spin- $\frac{3}{2}$ currents) in this paper does not exist in the orthogonal Wolf space coset theory. Compared to the unitary Wolf space coset, the orthogonal Wolf space coset has very simple nonzero structure constants and maybe this does not give the right current contents for the low spins $\left(s=1\right.$ or $s=\frac{3}{2}$ ). Other possibility comes from the fact that the regular conditions between the above candidate currents and the four spin- $\frac{1}{2}$ currents (and one spin-1 current) from the large $\mathcal{N}=4$ linear superconformal algebra restrict to 
their structures strongly. In other words, for low spin cases, there are too many conditions to solve for a few unknown terms. As the spin increases, one can consider more terms by multiplying the Kac-Moody currents and for the lowest higher spin-2 current one expects that there exists a unique solution for the regular conditions.

- The next 16 higher spin currents for general $N$

In order to see the spin dependence on the three-point function, sometimes it is not enough to obtain the three-point functions on two scalars with higher spin current living in the lowest $\mathcal{N}=4$ multiplet (3.1) only. Then one should look at the threepoint functions with higher spin current in the next $\mathcal{N}=4$ multiplet. For $N=3$, the higher spin current generating procedure is given in [41]. For the four higher spin- $\frac{5}{2}$ currents, the OPEs between the higher spin- 1 current and four higher spin- $\frac{5}{2}$ current found in this paper are needed. Once these new higher spin- $\frac{5}{2}$ currents are found, then the OPEs between the spin- $\frac{3}{2}$ currents living in the large $\mathcal{N}=4$ nonlinear superconformal algebra and them will determine the six higher spin-3 currents and one higher spin- 2 current. Furthermore, the four higher spin- $\frac{7}{2}$ currents can be fixed by the OPEs between the spin- $\frac{3}{2}$ currents and the above new higher spin-3 currents. Finally, the higher spin- 4 current can be determined by the OPE between the spin- $\frac{3}{2}$ current and the higher spin- $\frac{7}{2}$ current. In order to see the $N$ dependence on the OPEs, one should try to calculate them for low values for $N$. For the higher spin- 4 current, the first-order pole in (2.14) of [41] is rather complicated and it is nontrivial to obtain all the $N$ dependence in the first-order pole in there. As the spin increases, one should simplify the multiple products between the $d_{\bar{a} \bar{b}}^{\mu}$ tensor and the $h_{\bar{a} \bar{b}}^{\mu}$ tensor (as well as the structure constants) using some relevant identities. Otherwise, one cannot write down the higher spin currents in simple form.

- Oscillator formalism for the higher spin currents

Based on the previous work in [63, 64], the results in the $A d S_{3}$ bulk theory [4] were obtained for the higher spin-1 current and the four higher spin- $\frac{3}{2}$ currents as well as some currents in the large $\mathcal{N}=4$ nonlinear superconformal algebra. Then it is an open problem to obtain the oscillator formalism for the remaining higher spin currents (with general $N$ and $k$ ) by using the higher spin algebra in the $A d S_{3}$ bulk theory. In this approach, the nontrivial things are to write down all the higher spin fields in the primary basis.

- For the general $M$

So far, the $M$ in (1.1) is fixed as $M=2$. What happens for $M>2$ ? Along the lines of $[18,19]$, it is an open problem to study the coset model for $M>2$. Are there any supersymmetric coset models? It would be interesting to observe whether the $M=2$ case is special or not. 


\section{Acknowledgments}

This work was supported by the Mid-career Researcher Program through the National Research Foundation of Korea (NRF) grant funded by the Korean government (MEST) (No. 2012-045385/2013-056327/2014-051185). CA would like to thank the participants of the focus program of Asia Pacific Center for Theoretical Physics (APCTP) on "Liouville, Integrability and Branes (10) Focus Program at Asia-Pacific Center for Theoretical Physics", Sept. 03-14, 2014 for their feedbacks. CA appreciates APCTP for its hospitality during completion of this work. CA acknowledges warm hospitality from the School of Liberal Arts (and Institute of Convergence Fundamental Studies), Seoul National University of Science and Technology.

\section{A The generators of $\mathrm{SU}(N+2)$ in complex basis}

The $\mathrm{SU}(N+2)$ generators can be expressed in the complex (or Cartan-Weyl) basis. The index $a(2.3)$ is classified with $A$ and $A^{*}$. The $\left[(N+2)^{2}-1\right]$ generators of $\mathrm{SU}(N+2)$ are divided by the two sets of generators $T_{A}$ and $T_{A^{*}}$ where $A=1,2, \cdots, \frac{(N+2)^{2}-1}{2}$ with $N$ odd. Let us describe the $\frac{(N+2)^{2}-1}{2}$ unstarred generators $T_{A}$. There are $\frac{N+1}{2}$ diagonal generators and the remaining $\left[\frac{(N+2)^{2}-1}{2}-\frac{N+1}{2}\right]$ off-diagonal generators. For each off-diagonal matrix, the nonzero element (which is equal to 1 ) occurs once (at the specific row and column). Among those off-diagonal matrices, the nonzero elements of the $\frac{N(N-1)}{2}$ matrices are located at the lower half triangle matrices of the middle $N \times N$ matrices, one matrix has nonzero element with $(N+2)$-th row and first column and the remaining $2 N$ matrices have nonzero elements as follows:
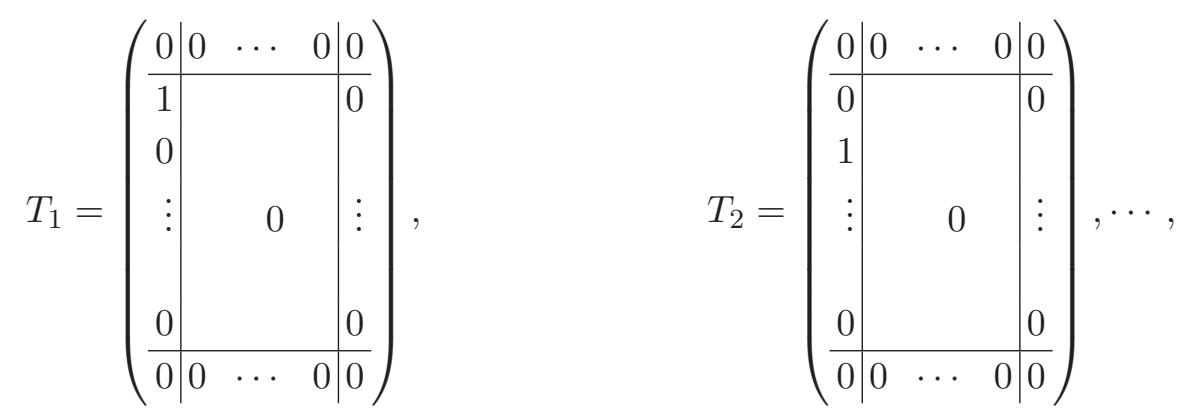

$$
T_{N}=\left(\begin{array}{c|ccc|c}
0 & 0 & \cdots & 0 & 0 \\
\hline 0 & & & & 0 \\
0 & & & & \\
\vdots & & 0 & \vdots \\
1 & & & & 0 \\
\hline 0 & 0 & \cdots & 0 & 0
\end{array}\right),
$$$$
T_{N+1}=\left(\begin{array}{c|ccc|c}
0 & 0 & \cdots & 0 & 0 \\
\hline 0 & & & & 0 \\
0 & & & & \\
\vdots & & 0 & \vdots \\
0 & & & & 0 \\
\hline 0 & 1 & \cdots & 0 & 0
\end{array}\right) \text {, }
$$ 


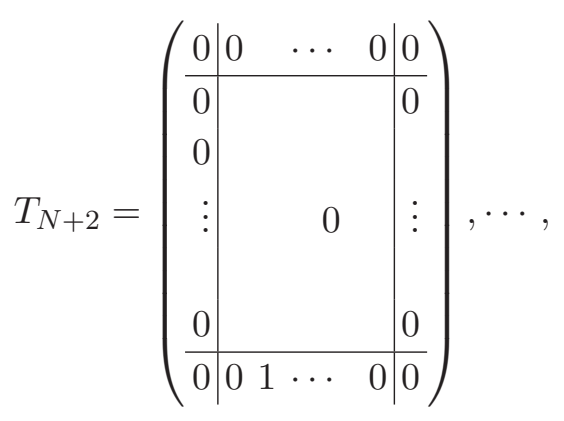

$$
T_{2 N}=\left(\begin{array}{r|rrr|r}
0 & 0 & \cdots & 0 & 0 \\
\hline 0 & & & & 0 \\
0 & & & \\
\vdots & & 0 & \vdots \\
0 & & & & 0 \\
\hline 0 & 0 & \cdots & 1 & 0
\end{array}\right) .
$$

That is, the matrix $T_{n}$ where $1 \leq n \leq N$ has nonzero element (numerical value is 1 ) at $(n+1)$-th row and first column (and other matrix elements vanish) and the matrix $T_{N+n}$ has nonzero element at $(N+2)$-th row and $(n+1)$-th column (and other matrix elements are vanishing). By interchanging of the role of both row and column (or transposing the matrix and taking the complex conjugation),

$$
T_{A^{*}} \equiv T_{A}^{\dagger},
$$

one obtains the other set of generators.

There exist $(N+1)$ Cartan generators in $\mathrm{SU}(N+2)$ denoted by $H_{1}, H_{2}, \cdots, H_{N+1}$. The Cartan generators are defined by [65]

$$
\left[H_{m}\right]_{i j}=\frac{1}{\sqrt{2 m(m+1)}}\left(\sum_{k=1}^{m} \delta_{i k} \delta_{j k}-m \delta_{i, m+1} \delta_{j, m+1}\right) .
$$

How does one construct these explicitly? The normalization is given in (A.3) and the diagonal element can be obtained as follows: one can start with $H_{1}$ by putting the number 1 at the first row and first column, put -1 at the next diagonal element and put the zeros at the remaining diagonal elements. For the $H_{m}$ where $1 \leq m \leq N+1$, one puts 1 at each diagonal element until $m$-th diagonal element, put $-m$ at the next diagonal element and put the zeros at the remaining diagonal elements. Then one obtains the following matrix representation for the Cartan generators as follows:

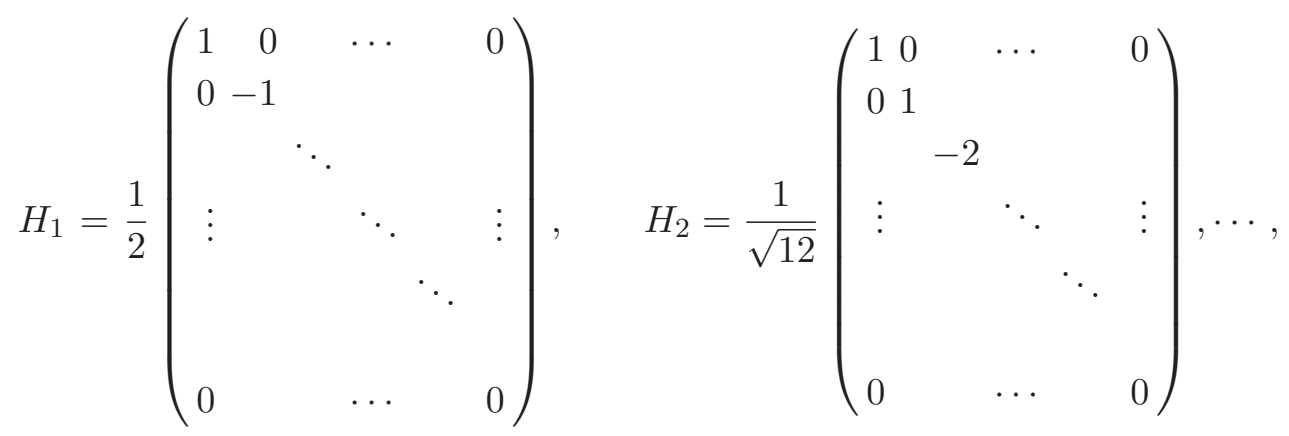




$$
H_{N+1}=\frac{1}{\sqrt{2(N+1)(N+2)}}\left(\begin{array}{ccccc}
1 & 0 & \cdots & & \\
0 & 1 & & & \\
& 1 & & & \\
\vdots & & \ddots & & \vdots \\
& & & \ddots & \\
& & & & \\
0 & & \cdots & & -(N+1)
\end{array}\right) .
$$

Because $N$ is odd, there are even number of Cartan generators. Then one can define the $\frac{N+1}{2}$ diagonal generators.

$$
\begin{gathered}
T_{p+1}=i H_{1}+H_{2}, \\
T_{p+2}=i H_{3}+H_{4}, \\
\vdots \\
T_{p+\frac{N+1}{2}}=i H_{N}+H_{N+1},
\end{gathered}
$$

where $p=\frac{(N+2)^{2}-1}{2}-\frac{N+1}{2}$. Half of these Cartan generators can be obtained from (A.2).

The metric is

$$
g_{a b}=\operatorname{Tr}\left(T_{a} T_{b}\right)=\left(\begin{array}{ll}
0 & 1 \\
1 & 0
\end{array}\right), \quad\left(a, b=1,2, \cdots,(N+2)^{2}-1\right) .
$$

This is consistent with the description of subsection 2.1. The nonvanishing metric components are

$$
g_{A A^{*}}=g_{A^{*} A}=1,
$$

where $A=1,2, \cdots, \frac{(N+2)^{2}-1}{2}$.

We have checked some OPEs and other relevant quantities for low values of $N$. The structure constants for those cases can be obtained from the generators in (A.1) and (A.4). One can also obtain the generators corresponding to the nonzero elements of the $\frac{N(N-1)}{2}$ matrices located at the lower half triangle matrices of the middle $N \times N$ matrices. For example, the structure constants containing the group $\mathrm{SU}(N+2)$ index appear in the spin-1 current (2.12) or spin-2 current (2.14). 


\section{B Eight rank-two tensors in the Wolf space coset}

The four rank two tensors $\left(12 \times 12\right.$ matrices) appearing in the spin- $\frac{3}{2}$ currents in $(2.5)$ can be generalized to the ones of $4 N \times 4 N$ matrices as follows:

$$
\begin{aligned}
& h_{\bar{a} \bar{b}}^{0} \equiv g_{\bar{a} \bar{b}}=\left(\begin{array}{cc|cc}
0 & 0 & 1 & 0 \\
0 & 0 & 0 & 1 \\
\hline 1 & 0 & 0 & 0 \\
0 & 1 & 0 & 0
\end{array}\right), h_{\bar{a} \bar{b}}^{1}=\left(\begin{array}{cc|cc}
0 & -i & 0 & 0 \\
i & 0 & 0 & 0 \\
\hline 0 & 0 & 0 & i \\
0 & 0 & -i & 0
\end{array}\right), \\
& h_{\bar{a} \bar{b}}^{2}=\left(\begin{array}{cc|cc}
0 & -1 & 0 & 0 \\
1 & 0 & 0 & 0 \\
\hline 0 & 0 & 0 & -1 \\
0 & 0 & 1 & 0
\end{array}\right), \\
& h_{\bar{a} \bar{b}}^{3} \equiv h_{\bar{a} \bar{c}}^{1} h_{\bar{b}}^{2 \bar{c}}=\left(\begin{array}{cc|cc}
0 & 0 & i & 0 \\
0 & 0 & 0 & i \\
\hline-i & 0 & 0 & 0 \\
0 & -i & 0 & 0
\end{array}\right),
\end{aligned}
$$

where each element in (B.1) is a $N \times N$ matrix. One can specify the indices further. The rows and columns of the first $2 N \times 2 N$ matrices are given by $\bar{A}$ and $\bar{A}$. The rows and columns of the first right $2 N \times 2 N$ matrices are given by $\bar{A}$ and $\bar{A}^{*}$. The rows and columns of the second left $2 N \times 2 N$ matrices are given by $\bar{A}^{*}$ and $\bar{A}$. The rows and columns of the second right $2 N \times 2 N$ matrices are given by $\bar{A}^{*}$ and $\bar{A}^{*}$. One also has

$$
\begin{aligned}
& i h_{\bar{a} \bar{b}}^{0}+h_{\bar{a} \bar{b}}^{3}=\left(\begin{array}{cc|cc}
0 & 0 & 2 i & 0 \\
0 & 0 & 0 & 2 i \\
\hline 0 & 0 & 0 & 0 \\
0 & 0 & 0 & 0
\end{array}\right), \quad i h_{\bar{a} \bar{b}}^{0}-h_{\bar{a} \bar{b}}^{3}=\left(\begin{array}{cc|c|cc}
0 & 0 & 0 & 0 \\
0 & 0 & 0 & 0 \\
\hline 2 i & 0 & 0 & 0 \\
0 & 2 i & 0 & 0
\end{array}\right), \\
& h_{\bar{a} \bar{b}}^{1}+i h_{\bar{a} \bar{b}}^{2}=\left(\begin{array}{cc|cc}
0 & 0 & 0 & 0 \\
0 & 0 & 0 & 0 \\
\hline 0 & 0 & 0 & -2 \\
0 & 0 & 2 & 0
\end{array}\right), \quad h_{\bar{a} \bar{b}}^{1}-i h_{\bar{a} \bar{b}}^{2}=\left(\begin{array}{cc|cc}
0 & -2 & 0 & 0 \\
2 & 0 & 0 & 0 \\
\hline 0 & 0 & 0 & 0 \\
0 & 0 & 0 & 0
\end{array}\right) .
\end{aligned}
$$

The rank two tensors defined in (3.16) (see also (3.11)) are generalized to the following $4 N \times 4 N$ matrices

$$
\begin{aligned}
d_{\bar{a} \bar{b}}^{0}=\left(\begin{array}{cc|cc}
0 & 0 & 1 & 0 \\
0 & 0 & 0 & -1 \\
\hline-1 & 0 & 0 & 0 \\
0 & 1 & 0 & 0
\end{array}\right), & d_{\bar{a} \bar{b}}^{1} \equiv d_{\bar{a}}^{0 \bar{c}} h_{\bar{c} \bar{b}}^{1}=\left(\begin{array}{cc|cc|c|c}
0 & -1 & 0 & 0 \\
-1 & 0 & 0 & 0 \\
\hline 0 & 0 & 0 & 1 \\
0 & 0 & 1 & 0
\end{array}\right), \\
d_{\bar{a} \bar{b}}^{2} \equiv d_{\bar{a}}^{0 \bar{c}} h_{\bar{c} \bar{b}}^{2}=\left(\begin{array}{cc|c|cc}
0 & -i & 0 & 0 \\
-i & 0 & 0 & 0 \\
\hline 0 & 0 & 0 & -i \\
0 & 0 & -i & 0
\end{array}\right), & d_{\bar{a} \bar{b}}^{3} \equiv d_{\bar{a}}^{0 \bar{c}} h_{\bar{c} \bar{b}}^{3}=\left(\begin{array}{cc|cc}
0 & 0 & i & 0 \\
0 & 0 & 0 & -i \\
\hline i & 0 & 0 & 0 \\
0 & -i & 0 & 0
\end{array}\right) .
\end{aligned}
$$

In (B.3), the above equations (B.1) are used. Compared to (B.1), the locations for the row and column appearing the nonzero elements in $d_{\bar{a} \bar{b}}^{\mu}$ are the same as the ones of $h_{\bar{a} \bar{b}}^{\mu}$. One 
also has

$$
\begin{aligned}
& i d_{\bar{a} \bar{b}}^{0}+d_{\bar{a} \bar{b}}^{3}=\left(\begin{array}{cc|cc}
0 & 0 & 2 i & 0 \\
0 & 0 & 0 & -2 i \\
\hline 0 & 0 & 0 & 0 \\
0 & 0 & 0 & 0
\end{array}\right), \quad i d_{\bar{a} \bar{b}}^{0}-d_{\bar{a} \bar{b}}^{3}=\left(\begin{array}{cc|cc}
0 & 0 & 0 & 0 \\
0 & 0 & 0 & 0 \\
\hline-2 i & 0 & 0 & 0 \\
0 & 2 i & 0 & 0
\end{array}\right), \\
& d_{\bar{a} \bar{b}}^{1}+i d_{\bar{a} \bar{b}}^{2}=\left(\begin{array}{cc|cc}
0 & 0 & 0 & 0 \\
0 & 0 & 0 & 0 \\
\hline 0 & 0 & 0 & 2 \\
0 & 0 & 2 & 0
\end{array}\right), \quad d_{\bar{a} \bar{b}}^{1}-i d_{\bar{a} \bar{b}}^{2}=\left(\begin{array}{cc|cc}
0 & -2 & 0 & 0 \\
-2 & 0 & 0 & 0 \\
\hline 0 & 0 & 0 & 0 \\
0 & 0 & 0 & 0
\end{array}\right) .
\end{aligned}
$$

It is easy to obtain eight tensors whose nonzero single element appears by taking a simple linear combination of (B.2) and (B.4). For example,

$$
-i h_{\bar{a} \bar{b}}^{0}-h_{\bar{a} \bar{b}}^{3}+i d_{\bar{a} \bar{b}}^{0}+d_{\bar{a} \bar{b}}^{3}=\left(\begin{array}{cc|cc}
0 & 0 & 0 & 0 \\
0 & 0 & 0 & -4 i \\
\hline 0 & 0 & 0 & 0 \\
0 & 0 & 0 & 0
\end{array}\right),
$$

which appears in the higher spin- $\frac{3}{2}$ current in (3.21).

\section{The large $\mathcal{N}=4$ nonlinear superconformal algebra}

In this appendix, we review the work of Van Proeyen [27]. The currents of $\mathcal{N}=4$ nonlinear superconformal algebra are given by one spin-2 current $L(z)$, four spin- $\frac{3}{2}$ currents $G^{\mu}(z)$, six spin-1 currents $A^{ \pm i}(z)$.

The spin-2 stress energy tensor satisfies the following OPE:

$$
L(z) L(w)=\frac{1}{(z-w)^{4}} \frac{\hat{c}}{2}+\frac{1}{(z-w)^{2}} 2 L(w)+\frac{1}{(z-w)} \partial L(w)+\cdots,
$$

where the central charge is given in (1.1)

$$
\hat{c}=\frac{6(k+1)(N+1)}{(k+N+2)}-3=\frac{3(k+N+2 k N)}{(k+N+2)},
$$

and the -3 in (C.1) is the contribution from the other terms in the modified stress energy tensor [40]. The above 10 currents are primary currents under the $L(z)$ as follows:

$$
\begin{aligned}
L(z) A^{ \pm i}(w) & =\frac{1}{(z-w)^{2}} A^{ \pm i}(w)+\frac{1}{(z-w)} \partial A^{ \pm i}(w)+\cdots \\
L(z) G^{\mu}(w) & =\frac{1}{(z-w)^{2}} \frac{3}{2} G^{\mu}(w)+\frac{1}{(z-w)} \partial G^{\mu}(w)+\cdots .
\end{aligned}
$$

The nontrivial OPEs between the four spin- $\frac{3}{2}$ currents can be summarized by

$$
\begin{aligned}
G^{\mu}(z) G^{\nu}(w)= & \frac{1}{(z-w)^{3}} \frac{2}{3} \delta^{\mu \nu} c_{\text {Wolf }}-\frac{1}{(z-w)^{2}} \frac{8}{(k+N+2)}\left(N \alpha_{\mu \nu}^{+i} A_{i}^{+}+k \alpha_{\mu \nu}^{-i} A_{i}^{-}\right)(w) \\
& +\frac{1}{(z-w)}\left[2 \delta^{\mu \nu} L-\frac{4}{(k+N+2)} \partial\left(N \alpha_{\mu \nu}^{+i} A_{i}^{+}+k \alpha_{\mu \nu}^{-i} A_{i}^{-}\right)\right. \\
& \left.-\frac{8}{(k+N+2)}\left(\alpha^{+i} A_{i}^{+}-\alpha^{-i} A_{i}^{-}\right)_{\rho(\mu}\left(\alpha^{+j} A_{j}^{+}-\alpha^{-j} A_{j}^{-}\right)_{\nu)}{ }^{\rho}\right](w)+\cdots,
\end{aligned}
$$


where the indices run over $\mu, \nu, \rho=0,1,2,3$ and $i, j=1,2,3$. The OPE (C.2) is used in section 2. The Wolf space coset central charge is given in (1.1)

$$
c_{\text {Wolf }}=\frac{6 k N}{(2+k+N)} .
$$

The $\alpha^{ \pm i}$ is defined by

$$
\alpha_{\mu \nu}^{ \pm i}=\frac{1}{2}\left( \pm \delta_{i \mu} \delta_{\nu 0} \mp \delta_{i \nu} \delta_{\mu 0}+\epsilon_{i \mu \nu}\right)
$$

The OPE between the six spin- 1 currents and the four spin- $\frac{3}{2}$ currents (related to the discussion at the end of section 2) is

$$
A^{ \pm i}(z) G^{\mu}(w)=\frac{1}{(z-w)} \alpha_{\mu \nu}^{ \pm i} G^{\nu}(w)+\cdots
$$

Finally, the OPEs between the spin- 1 currents are given by

$$
A^{ \pm i}(z) A^{ \pm j}(w)=-\frac{1}{(z-w)^{2}} \frac{1}{2} \delta^{i j} \hat{k}^{ \pm}+\frac{1}{(z-w)} \epsilon^{i j k} A^{ \pm k}(w)+\cdots,
$$

where $\hat{k}^{+}=k$ and $\hat{k}^{-}=N$ are the levels associated with each group in $\mathrm{SU}(2)_{\hat{k}^{+}} \times \mathrm{SU}(2)_{\hat{k}^{-}}$ respectively.

Let us present (C.2) explicitly as follows:

$$
\begin{aligned}
G^{0}(z) G^{0}(w)= & \frac{1}{(z-w)^{3}} \frac{2}{3} c_{\text {Wolf }}+\frac{1}{(z-w)}\left[2 L+\frac{2}{(k+N+2)} \sum_{i=1}^{3}\left(A^{+i}+A^{-i}\right)^{2}\right](w)+\cdots, \\
G^{i}(z) G^{i}(w)= & \frac{1}{(z-w)^{3}} \frac{2}{3} c_{\text {Wolf }}+\frac{1}{(z-w)}[2 L \\
& \left.+\frac{2}{(k+N+2)} \sum_{j=1}^{3}\left(A^{+j}+a(i, j) A^{-j}\right)^{2}\right](w)+\cdots, \quad(i=1,2,3), \\
G^{0}(z) G^{i}(w)= & \frac{4}{(k+N+2)}\left[\frac{1}{(z-w)^{2}}\left(N A^{+i}-k A^{-i}\right)\right. \\
& \left.+\frac{1}{(z-w)}\left(\frac{1}{2} \partial\left(N A^{+i}-k A^{-i}\right)+\sum_{j, k=1}^{3} \epsilon^{i j k} A^{+j} A^{-k}\right)\right](w)+\cdots, \quad(i=1,2,3) \\
G^{i}(z) G^{j}(w)= & -\frac{4}{(k+N+2)}\left[\frac{1}{(z-w)^{2}} \sum_{k=1}^{3} \epsilon^{i j k}\left(N A^{+k}+k A^{-k}\right)\right. \\
& \left.+\frac{1}{(z-w)}\left(\frac{1}{2} \sum_{k=1}^{3} \epsilon^{i j k} \partial\left(N A^{+k}+k A^{-k}\right)-\left(A^{+i} A^{-j}+A^{-i} A^{+j}\right)\right)\right](w)+\cdots,
\end{aligned}
$$

where the indices are $(i, j)=(1,2),(2,3)$ or $(3,1)$ in the last OPE of (C.4). The following index appearing in the second OPE of (C.4) occurs in [27]

$$
a(i, j) \equiv\left\{\begin{array}{l}
+1 \text { if } i=j \\
-1 \text { if } i \neq j
\end{array} .\right.
$$


The second equation in (C.4) has the following decomposition with (C.5)

$$
\begin{aligned}
L(z)= & \frac{1}{2(k+N+2)^{2}}\left[(k+N+2) V_{\bar{a}} V^{\bar{a}}+k Q_{\bar{a}} \partial Q^{\bar{a}}+h_{\bar{a} \bar{b}}^{i} h_{\bar{c} \bar{d}}^{i} f_{e}^{\bar{b} \bar{d}} Q^{\bar{a}} Q^{\bar{c}} V^{e}\right](z) \\
& -\frac{1}{(k+N+2)} \sum_{j=1}^{3}\left(A^{+j}+a(i, j) A^{-j}\right)^{2}(z), \quad(i=1,2,3), \\
\equiv & L_{W}^{i}(z)+L_{\mathrm{SU}(2)}^{i}(z) .
\end{aligned}
$$

Similarly, one can write $L(z)$ in terms of the sum of $L_{W}^{0}(z)$ and $L_{\mathrm{SU}(2)}^{0}(z)$ using the first equation of (C.4). With the index $a(0, j) \equiv 1$, one has the following decomposition

$$
\begin{aligned}
L_{W}^{\mu}(z) & \equiv \frac{1}{2(k+N+2)^{2}}\left[(k+N+2) V_{\bar{a}} V^{\bar{a}}+k Q_{\bar{a}} \partial Q^{\bar{a}}+h_{\bar{a} \bar{b}}^{\mu} h_{\bar{c} \bar{d}}^{\mu} f^{\bar{b} \bar{d}} Q_{e}^{\bar{a}} Q^{\bar{c}} V^{e}\right](z), \\
L_{\mathrm{SU}(2)}^{\mu}(z) & \equiv-\frac{1}{(k+N+2)} \sum_{j=1}^{3}\left(A^{+j}+a(\mu, j) A^{-j}\right)^{2}(z), \quad(\mu=0,1,2,3),
\end{aligned}
$$

where there is no sum over the index $\mu$. Therefore, there are four different $L_{W}^{\mu}(z)$ and four different $L_{\mathrm{SU}(2)}^{\mu}(z)$. However, the sum of each $L_{W}^{\mu}(z)$ and each $L_{\mathrm{SU}(2)}^{\mu}(z)$ leads to a single $L(z)$. In other words, for each $\mu$ index,

$$
L(z)=L_{W}^{\mu}(z)+L_{\mathrm{SU}(2)}^{\mu}(z) .
$$

One can also check the following OPEs between $L_{W}^{\mu}(z)$ and $L_{\mathrm{SU}(2)}^{\mu}(z)$ as follows:

$$
\begin{aligned}
L_{W}^{\mu}(z) L_{W}^{\mu}(w) & =\frac{1}{(z-w)^{4}} \frac{{ }^{c} \frac{\mathrm{Wolf}}{2}+\frac{1}{(z-w)^{2}} 2 L_{W}^{\mu}(w)+\frac{1}{(z-w)} \partial L_{W}^{\mu}(w)+\cdots,}{L_{\mathrm{SU}(2)}^{\mu}(z) L_{\mathrm{SU}(2)}^{\mu}(w)}=\frac{1}{(z-w)^{4}} \frac{{ }_{\mathrm{SU}(2)}}{2}+\frac{1}{(z-w)^{2}} 2 L_{\mathrm{SU}(2)}^{\mu}(w)+\frac{1}{(z-w)} \partial L_{\mathrm{SU}(2)}^{\mu}(w)+\cdots, \\
L_{W}^{\mu}(z) L_{\mathrm{SU}(2)}^{\mu}(w) & =+\cdots
\end{aligned}
$$

From (C.6), one can check that the central charge in (1.1) with (C.3) is given by

$$
\hat{c}=c_{\mathrm{Wolf}}+c_{\mathrm{SU}(2)}, \quad c_{\mathrm{SU}(2)}=\frac{3(k+N)}{(k+N+2)} .
$$

Furthermore, each spin- $\frac{3}{2}$ current $G^{\mu}(w)$ is a primary current under the corresponding $L_{W}^{\mu}(z)$ and the sum of $\left(A^{+i}+a(\mu, i) A^{-i}\right)(w)$ is primary current under the $L_{\mathrm{SU}(2)}^{\mu}(z)$ stress energy tensor as follows:

$$
\begin{aligned}
L_{W}^{\mu}(z) G^{\mu}(w)= & \frac{1}{(z-w)^{2}} \frac{3}{2} G^{\mu}(w)+\frac{1}{(z-w)} \partial G^{\mu}(w)+\cdots, \\
L_{\mathrm{SU}(2)}^{\mu}(z)\left(A^{+i}+a(\mu, i) A^{-i}\right)(w)= & \frac{1}{(z-w)^{2}}\left(A^{+i}+a(\mu, i) A^{-i}\right)(w) \\
& +\frac{1}{(z-w)} \partial\left(A^{+i}+a(\mu, i) A^{-i}\right)(w)+\cdots .
\end{aligned}
$$

Note that the current $\left(A^{+i}+a(\mu, i) A^{-i}\right)(w)$ has a level $(k+N)$ which is the sum of $\hat{k}^{+}$ and $\hat{k}^{-}$because the currents $A^{+i}(z)$ and the currents $A^{-i}(w)$ commute with each other. Moreover, $L_{\mathrm{SU}(2)}^{\mu}(z) G^{\mu}(w)=+\cdots$ and $L_{W}^{\mu}(z)\left(A^{+i}+a(\mu, i) A^{-i}\right)(w)=+\cdots$. 


\section{The OPEs between the spin- $\frac{3}{2}$ currents for general $N$ in the bispinor notation}

We present the OPEs between the spin- $\frac{3}{2}$ currents for general $N$ in the bispinor notation (the corresponding equation in $N=3$ appears in (A.3) of [40]) as follows:

$$
\begin{aligned}
& \hat{G}_{11}(z) \hat{G}_{11}(w)=\frac{1}{(z-w)} \frac{4}{(N+k+2)}\left[-\hat{A}_{+} \hat{B}_{-}\right](w)+\cdots, \\
& \hat{G}_{11}(z) \hat{G}_{12}(w)=\frac{1}{(z-w)^{2}}\left[4 i \gamma_{A} \hat{A}_{+}\right](w)+\frac{1}{(z-w)}\left[2 i \gamma_{A} \partial \hat{A}_{+}+\frac{4}{(N+k+2)} \hat{A}_{+} \hat{B}_{3}\right](w)+\cdots, \\
& \hat{G}_{11}(z) \hat{G}_{21}(w)=\frac{1}{(z-w)^{2}}\left[-4 i \gamma_{B} \hat{B}_{-}\right](w)+\frac{1}{(z-w)}\left[-2 i \gamma_{B} \partial \hat{B}_{-}+\frac{4}{(N+k+2)} \hat{A}_{3} \hat{B}_{-}\right](w) \\
& +\cdots \text {, } \\
& \hat{G}_{11}(z) \hat{G}_{22}(w)=\frac{1}{(z-w)^{3}} \frac{2}{3} c_{\text {Wolf }}+\frac{1}{(z-w)^{2}}\left[4 i\left(\gamma_{A} \hat{A}_{3}-\gamma_{B} \hat{B}_{3}\right)\right](w)+\frac{1}{(z-w)}[2 \hat{T} \\
& \left.+2 i \partial\left(\gamma_{A} \hat{A}_{3}-\gamma_{B} \hat{B}_{3}\right)+\frac{2}{(k+N+2)}\left(\hat{A}_{i} \hat{A}_{i}+\hat{B}_{i} \hat{B}_{i}+2 \hat{A}_{3} \hat{B}_{3}\right)\right](w)+\cdots, \\
& \hat{G}_{12}(z) \hat{G}_{12}(w)=\frac{1}{(z-w)} \frac{4}{(N+k+2)}\left[\hat{A}_{+} \hat{B}_{+}\right](w)+\cdots, \\
& \hat{G}_{12}(z) \hat{G}_{21}(w)=\frac{1}{(z-w)^{3}} \frac{2}{3} c_{\text {Wolf }}+\frac{1}{(z-w)^{2}}\left[4 i\left(\gamma_{A} \hat{A}_{3}+\gamma_{B} \hat{B}_{3}\right)\right](w)+\frac{1}{(z-w)}[2 \hat{T} \\
& \left.+2 i \partial\left(\gamma_{A} \hat{A}_{3}+\gamma_{B} \hat{B}_{3}\right)+\frac{2}{(k+N+2)}\left(\hat{A}_{i} \hat{A}_{i}+\hat{B}_{i} \hat{B}_{i}-2 \hat{A}_{3} \hat{B}_{3}\right)\right](w)+\cdots, \\
& \hat{G}_{12}(z) \hat{G}_{22}(w)=\frac{1}{(z-w)^{2}}\left[-4 i \gamma_{B} \hat{B}_{+}\right](w)+\frac{1}{(z-w)}\left[-2 i \gamma_{B} \partial \hat{B}_{+}+\frac{4}{(N+k+2)} \hat{A}_{3} \hat{B}_{+}\right](w) \\
& +\cdots \text {, } \\
& \hat{G}_{21}(z) \hat{G}_{21}(w)=\frac{1}{(z-w)} \frac{4}{(N+k+2)}\left[\hat{A}_{-} \hat{B}_{-}\right](w)+\cdots, \\
& \hat{G}_{21}(z) \hat{G}_{22}(w)=\frac{1}{(z-w)^{2}}\left[4 i \gamma_{A} \hat{A}_{-}\right](w)+\frac{1}{(z-w)}\left[2 i \gamma_{A} \partial \hat{A}_{-}+\frac{4}{(N+k+2)} \hat{A}_{-} \hat{B}_{3}\right](w) \\
& +\cdots \text {, } \\
& \hat{G}_{22}(z) \hat{G}_{22}(w)=\frac{1}{(z-w)} \frac{4}{(N+k+2)}\left[-\hat{A}_{-} \hat{B}_{+}\right](w)+\cdots,
\end{aligned}
$$

where $\gamma_{A} \equiv \frac{N}{N+k+2}$ and $\gamma_{B} \equiv \frac{k}{N+k+2}$. One can express these by one single equation as done in (C.2) but instead of doing this, one presents them in components. These OPEs (D.1) can be used when one calculates the higher spin-3 current relevant to the term of the first order pole in the OPE $\hat{G}_{21}(z) \partial \hat{G}_{12}(w)$ (and they are used in many other places). 


\section{E The identities from the cubic terms in the first-order pole of the OPE between the spin- $\frac{3}{2}$ currents in $(2.6)$}

There exist the following relations in the cubic terms in the first-order pole of the OPE between the spin- $\frac{3}{2}$ currents in $(2.6)$

$$
\begin{aligned}
& 2 N f^{\bar{a} \bar{b}}{ }_{e} h_{\bar{a}[\bar{c}}^{0} h_{\bar{d}] \bar{b}}^{1}=f^{\bar{a} \bar{b}} h_{\bar{a} \bar{b}}^{[2} h_{\bar{c} \bar{d}}^{3]}, \\
& 2 N f^{\bar{a} \bar{b}}{ }_{e} h_{\bar{a}[\bar{c}}^{0} h_{\bar{d}] \bar{b}}^{2}=f^{\bar{a} \bar{b}}{ }_{e} h_{\bar{a} \bar{b}}^{[3} h_{\bar{c} \bar{d}}^{1]}, \\
& 2 N f^{\bar{a} \bar{b}}{ }_{e} h_{\bar{a}[\bar{c}}^{0} h_{\bar{d}] \bar{b}}^{3}=f^{\bar{a} \bar{b}}{ }_{e} h_{\bar{a} \bar{b}}^{[1} h_{\bar{c} \bar{d}}^{2]}, \\
& 2 N f^{\bar{a} \bar{b}}{ }_{e} h_{\bar{a}[\bar{c}}^{1} h_{\bar{d}] \bar{b}}^{2}=f^{\bar{a} \bar{b}} h_{\bar{a} \bar{b}}^{(1} h_{\bar{d} \bar{c}}^{2)}, \\
& 2 N f^{\bar{a} \bar{b}}{ }_{e} h_{\bar{a}[\bar{c}}^{2} h_{\bar{d}] \bar{b}}^{3}=f^{\bar{a} \bar{b}} h_{\bar{a} \bar{b}}^{(2} h_{\bar{d} \bar{c}}^{3)}, \\
& 2 N f^{\bar{a} \bar{b}}{ }_{e} h_{\bar{a}[\bar{c}}^{3} h_{\bar{d}] \bar{b}}^{1}=f^{\bar{a} \bar{b}}{ }_{e} h_{\bar{a} \bar{b}}^{(3} h_{\bar{d} \bar{c}}^{1)}, \\
& 2 N f^{\bar{a} \bar{b}}{ }_{e}\left(h_{\bar{a} \bar{c}}^{0} h_{\bar{b} \bar{d}}^{0}-h_{\bar{a} \bar{c}}^{1} h_{\bar{b} \bar{d}}^{1}\right)=f^{\bar{a} \bar{b}}{ }_{e}\left(h_{\bar{a} \bar{b}}^{2} h_{\bar{c} \bar{d}}^{2}+h_{\bar{a} \bar{b}}^{3} h_{\bar{c} \bar{d}}^{3}\right), \\
& 2 N f_{e}^{\bar{a} \bar{b}}\left(h_{\bar{a} \bar{c}}^{0} h_{\bar{b} \bar{d}}^{0}-h_{\bar{a} \bar{c}}^{2} h_{\bar{b} \bar{d}}^{2}\right)=f_{e}^{\bar{a} \bar{b}}\left(h_{\bar{a} \bar{b}}^{3} h_{\bar{c} \bar{d}}^{3}+h_{\bar{a} \bar{b}}^{1} h_{\bar{c} \bar{d}}^{1}\right) \text {, } \\
& 2 N f^{\bar{a} \bar{b}}{ }_{e}\left(h_{\bar{a} \bar{c}}^{0} h_{\bar{b} \bar{d}}^{0}-h_{\bar{a} \bar{c}}^{3} h_{\bar{b} \bar{d}}^{3}\right)=f^{\bar{a} \bar{b}}\left(h_{\bar{a} \bar{b}}^{1} h_{\bar{c} \bar{d}}^{1}+h_{\bar{a} \bar{b}}^{2} h_{\bar{c} \bar{d}}^{2}\right) .
\end{aligned}
$$

The left hand side corresponds to the cubic term of the first order pole in (2.6) while the right hand side corresponds to the same cubic term of the first order pole in (C.2).

In the bispinor notation, from the relations of (2.16) and (D.1), the above relations (E.1) lead to the following equations

$$
\begin{aligned}
& f^{\bar{a} \bar{b}}{ }_{e}\left(i g+h^{3}\right)_{\bar{a}[\bar{c}}\left(i g-h^{3}\right)_{\bar{d}] \bar{b}}=-\frac{1}{2 N} f_{e}^{\bar{a} \bar{b}}\left(h^{1}-i h^{2}\right)_{\bar{a} \bar{b}}\left(h^{1}+i h^{2}\right)_{\bar{c} \bar{d}}, \\
& f^{\bar{a} \bar{b}}{ }_{e}\left(i g+h^{3}\right)_{\bar{a}[\bar{c}}\left(i g+h^{3}\right)_{\bar{d}] \bar{b}}=\frac{1}{2 N} f^{\bar{a} \bar{b}}{ }_{e}\left(h_{\bar{a} \bar{b}}^{1} h_{\bar{c} \bar{d}}^{1}+h_{\bar{a} \bar{b}}^{2} h_{\bar{c} \bar{d}}^{2}\right)-2 f_{\bar{c} \bar{d} e}, \\
& f^{\bar{a} \bar{a}}{ }_{e}\left(i g+h^{3}\right)_{\bar{a}[\bar{c}}\left(h^{1}-i h^{2}\right)_{\bar{d}] \bar{b}}=-\frac{1}{2 N} f^{\bar{a} \bar{b}}{ }_{e}\left(h^{1}-i h^{2}\right)_{\bar{a} \bar{b}} h_{\bar{c} \bar{d}}^{3}, \\
& f^{\bar{a} \bar{b}}{ }_{e}\left(i g+h^{3}\right)_{\bar{a}[\bar{c}}\left(h^{1}+i h^{2}\right)_{\bar{d}] \bar{b}}=-\frac{1}{2 N} f^{\bar{a} \bar{b}}{ }_{e} h_{\bar{a} \bar{b}}^{3}\left(h^{1}+i h^{2}\right)_{\bar{c} \bar{d}}, \\
& f^{\bar{a} \bar{b}}{ }_{e}\left(i g-h^{3}\right)_{\bar{a}[\bar{c}}\left(i g+h^{3}\right)_{\bar{d}] \bar{b}}=-\frac{1}{2 N} f_{e}^{\bar{a} \bar{b}}\left(h^{1}+i h^{2}\right)_{\bar{a} \bar{b}}\left(h^{1}-i h^{2}\right)_{\bar{c} \bar{d}}, \\
& f_{e}^{\bar{a} \bar{b}}\left(i g-h^{3}\right)_{\bar{a}[\bar{c}}\left(h^{1}-i h^{2}\right)_{\bar{d}] \bar{b}}=\frac{1}{2 N} f^{\bar{a} \bar{b}}{ }_{e} h_{\bar{a} \bar{b}}^{3}\left(h^{1}-i h^{2}\right)_{\bar{c} \bar{d}}, \\
& f^{\bar{a} \bar{b}}{ }_{e}\left(i g-h^{3}\right)_{\bar{a}[\bar{c}}\left(h^{1}+i h^{2}\right)_{\bar{d}] \bar{b}}=\frac{1}{2 N} f_{e}^{\bar{a} \bar{b}}\left(h^{1}+i h^{2}\right)_{\bar{a} \bar{b}} h_{\bar{c} \bar{d}}^{3}, \\
& f^{\bar{a} \bar{b}}{ }_{e}\left(h^{1}-i h^{2}\right)_{\bar{a}[\bar{c}}\left(h^{1}-i h^{2}\right)_{\bar{d}] \bar{b}}=-\frac{1}{2 N} f_{e}^{\bar{a} \bar{b}}\left(h^{1}-i h^{2}\right)_{\bar{a} \bar{b}}\left(h^{1}-i h^{2}\right)_{\bar{c} \bar{d}} \text {, } \\
& f_{e}^{\bar{a} \bar{b}}{ }_{e}\left(h^{1}-i h^{2}\right)_{\bar{a}[\bar{c}}\left(h^{1}+i h^{2}\right)_{\bar{d}] \bar{b}}=\frac{1}{2 N} f^{\bar{a} \bar{b}}{ }_{e}\left(h_{\bar{a} \bar{b}}^{1} h_{\bar{c} \bar{d}}^{1}+h_{\bar{a} \bar{b}}^{2} h_{\bar{c} \bar{d}}^{2}+2 h_{\bar{a} \bar{b}}^{3} h_{\bar{c} \bar{d}}^{3}\right)-2 f_{\bar{c} \bar{d} e} \text {, } \\
& f^{\bar{a} \bar{b}}{ }_{e}\left(h^{1}+i h^{2}\right)_{\bar{a}[\bar{c}}\left(h^{1}+i h^{2}\right)_{\bar{d}] \bar{b}}=-\frac{1}{2 N} f_{e}^{\bar{a} \bar{b}}\left(h^{1}+i h^{2}\right)_{\bar{a} \bar{b}}\left(h^{1}+i h^{2}\right)_{\bar{c} \bar{d}} .
\end{aligned}
$$


From the cubic terms in the first-order pole of $G^{\mu}(z) G^{\prime \mu}(w)$ (for $N=3,5,7$ ) one has the following relation where there is no sum over the index $\mu$

$$
h_{\bar{a} \bar{b}}^{\mu} d_{\bar{c} \bar{d}}^{\mu} f_{e}^{\bar{b} \bar{d}}=h_{\bar{c} \bar{b}}^{\mu} d_{\bar{a} \bar{d}}^{\mu} f_{e}^{\bar{b} \bar{d}} \quad(\mu=0,1,2,3) .
$$

One can prove the identities (E.2) using the second property in (3.12).

From the OPE $G_{m n}(z) G_{m n}^{\prime}(w)$ (for $N=3,5,7$ ), the following identities hold

$$
\begin{aligned}
& f^{\bar{a} \bar{b}}\left(i g+h^{3}\right)_{\bar{a}[\bar{c}}\left(d^{1}-i d^{2}\right)_{\bar{d}] \bar{b}}=-\frac{i}{2 N} f_{e}^{\bar{a} \bar{b}}\left(h^{1}-i h^{2}\right)_{\bar{a} \bar{b}} d_{\bar{c} \bar{d}}^{0}, \\
& f_{e}^{\bar{a} \bar{b}}\left(i g-h^{3}\right)_{\bar{a}[\bar{c}}\left(d^{1}+i d^{2}\right)_{\bar{d}] \bar{b}}=-\frac{i}{2 N} f_{e}^{\bar{a} \bar{b}}\left(h^{1}+i h^{2}\right)_{\bar{a} \bar{b} \bar{b}} d_{\bar{c} \bar{d}}^{0}, \\
& f^{\bar{a} \bar{b}}{ }_{e}\left(h^{1}-i h^{2}\right)_{\bar{a}[\bar{c}}\left(i d^{0}-d^{3}\right)_{\bar{d}] \bar{b}}=-\frac{i}{2 N} f_{e}^{\bar{a} \bar{b}}\left(h^{1}-i h^{2}\right)_{\bar{a} \bar{b}} d_{\bar{c} \bar{d}}^{0}, \\
& f^{\bar{a} \bar{a}}{ }_{e}\left(h^{1}+i h^{2}\right)_{\bar{a}[\bar{c}}\left(i d^{0}+d^{3}\right)_{\bar{d}] \bar{b}}=-\frac{i}{2 N} f_{e}^{\bar{a} \bar{b}}\left(h^{1}+i h^{2}\right)_{\bar{a} \bar{b}} d_{\bar{c} \bar{d}}^{0}, \\
& f^{\bar{a} \bar{b}}\left(i g+h^{3}\right)_{\bar{a}[\bar{c}}\left(i d^{0}-d^{3}\right)_{\bar{d}] \bar{b}}=0, \\
& f^{\bar{a} \bar{b}}{ }_{e}\left(i g-h^{3}\right)_{\bar{a}[\bar{c}}\left(i d^{0}+d^{3}\right)_{\bar{d}] \bar{b}}=0, \\
& f^{\bar{a} \bar{b}}{ }_{e}\left(h^{1}-i h^{2}\right)_{\bar{a}[\bar{c}}\left(d^{1}-i d^{2}\right)_{\bar{d}] \bar{b}}=0, \\
& \left.f_{e}^{\bar{a} \bar{b}}\left(h^{1}+i h^{2}\right)_{\bar{a}[\bar{c}}\left(d^{1}+i d^{2}\right) \bar{d}\right] \bar{b}=0 .
\end{aligned}
$$

These are used in (3.28) and (3.30).

\section{F Some OPEs and identities relevant to the higher spin- $\frac{5}{2}$ currents}

For the higher spin- $\frac{5}{2}$ currents, one should calculate the spin- $\frac{3}{2}$ currents and the spin2 currents. The former has $Q^{\bar{a}} V^{\bar{b}}(z)$ and the latter contains the two quadratic terms, $V^{\bar{c}} V^{\bar{d}}(w)$ and $Q^{\bar{c}} \partial Q^{\bar{d}}(w)$, and the cubic term $Q^{\bar{c}} Q^{\bar{d}} V^{e}(w)$. It turns out that the first order poles of the corresponding OPEs, which will be used in the subsection 3.4, are given by

$$
\begin{aligned}
\left.Q^{\bar{a}} V^{\bar{b}}(z) V^{\bar{c}} V^{\bar{d}}(w)\right|_{\frac{1}{(z-w)}=} & {\left[k\left(g^{\bar{c} \bar{b}} \partial Q^{\bar{a}} V^{\bar{d}}+g^{\overline{d \bar{b}}} \partial Q^{\bar{a}} V^{\bar{c}}\right)+f_{e}^{\bar{c} \bar{b}} Q^{\bar{a}} V^{\bar{d}} V^{e}\right.} \\
& \left.+f_{e}^{\overline{d \bar{b}}} Q^{\bar{a}} V^{\bar{c}} V^{e}+f_{e}^{\bar{c} \bar{b}} f^{\bar{d} e}{ }_{\bar{f}} \partial\left(Q^{\bar{a}} V^{\bar{f}}\right)\right](w), \\
\left.Q^{\bar{a}} V^{\bar{b}}(z) Q^{\bar{c}} \partial Q^{\bar{d}}(w)\right|_{\frac{1}{(z-w)}=} & (k+N+2)\left(g^{\bar{d} \bar{a}} Q^{\bar{c}} \partial V^{\bar{b}}-g^{\bar{c} \bar{a}} \partial Q^{\bar{d}} V^{\bar{b}}\right)(w), \\
\left.Q^{\bar{a}} V^{\bar{b}}(z) Q^{\bar{c}} Q^{\bar{d}} V^{e}(w)\right|_{\frac{1}{(z-w)}=}= & (k+N+2)\left(g^{\bar{d} \bar{a}} Q^{\bar{c}} V^{\bar{b}} V^{e}-g^{\bar{c} \bar{a}} Q^{\bar{d}} V^{\bar{b}} V^{e}\right) \\
& \left.+k g^{e \bar{b}} Q^{\bar{c}} Q^{\bar{d}} \partial Q^{\bar{a}}+f_{f}^{e \bar{b}} Q^{\bar{c}} Q^{\bar{d}} Q^{\bar{a}} V^{f}\right](w) .
\end{aligned}
$$

Therefore, the final higher spin- $\frac{5}{2}$ currents can be obtained by multiplying $h_{\bar{a} \bar{b}}^{\mu}$ and other relevant rank two and three tensors (appearing in the spin-2 currents) in (F.1). The spin-2 currents have the composite terms from the currents of large $\mathcal{N}=4$ nonlinear superconformal algebra. One obtains the contributions from the OPEs between the spin- $\frac{3}{2}$ currents and those composite terms without any difficulty because they are part of large $\mathcal{N}=4$ nonlinear superconformal algebra (and their descendants). 
One presents further simplifications as follows (for $N=3,5,7$ ):

$$
\begin{aligned}
\left(i g+h^{3}\right)_{\bar{a} \bar{b}}\left(i d^{1}+d^{2}\right)_{\bar{c} \bar{d}} f^{\bar{b} \bar{c}}{ }_{e} f^{\bar{d} e_{\bar{f}}} \partial\left(Q^{\bar{a}} V^{\bar{f}}\right) & =2 N\left(d^{1}-i d^{2}\right)_{\bar{a} \bar{b}} \partial\left(Q^{\bar{a}} V^{\bar{b}}\right), \\
\left(i g+h^{3}\right)_{\bar{a} \bar{b}}\left(d^{0}-i d^{3}\right)_{\bar{c} \bar{d}} f_{e}^{\bar{b} \bar{c}} f_{e}^{e \bar{d}}{ }_{\bar{f}} \partial\left(Q^{\bar{a}} V^{\bar{f}}\right) & =2 i(N+1)\left(d^{0}-i d^{3}\right)_{\bar{a} \bar{b}} \partial\left(Q^{\bar{a}} V^{\bar{b}}\right), \\
\left(i g-h^{3}\right)_{\bar{a} \bar{b}}\left(d^{0}-i d^{3}\right)_{\bar{c} \bar{d}} f^{\bar{b} \bar{c}}{ }_{e} f^{e \bar{d}}{ }_{\bar{f}} \partial\left(Q^{\bar{a}} V^{\bar{f}}\right) & =2\left(i d^{0}-d^{3}\right)_{\bar{a} \bar{b}} \partial\left(Q^{\bar{a}} V^{\bar{b}}\right) .
\end{aligned}
$$

For example, the first relation in (F.2) comes from the equation (3.46). The spin- $\frac{3}{2}$ current $\hat{G}_{21}(z)$ has a term $\left(h^{3}+i g\right)_{\bar{a} \bar{b}}$ and the higher spin-2 current has $\left(i d^{1}+d^{2}\right)_{\bar{c} \bar{d}}$ in the quadratic term of (3.27). Moreover, according to the first equation in (F.1), the quadratic structure constant term has total derivative term. Combining these factors leads to the left hand side of the first equation of (F.2). The right hand side appears in the final expression of the higher spin- $\frac{5}{2}$ current given in (3.46).

There are other types of identities as follows (for $N=3,5$ ):

$$
\begin{aligned}
& \left(i g+h^{3}\right)_{\bar{a} \bar{b}}\left(h^{1}-i h^{2}\right)_{\bar{e} \bar{f}}\left(i d^{0}-d^{3}\right)_{\bar{c} \bar{g}} f_{d}^{\bar{f} \bar{g}} f_{{ }_{\bar{h}}}^{d \bar{b}} Q^{\bar{e}} Q^{\bar{c}} Q^{\bar{a}} V^{\bar{h}}=2 d_{\bar{a} \bar{b}}^{0}\left(h^{1}-i h^{2}\right)_{\bar{c} \bar{d}} Q^{\bar{a}} Q^{\bar{b}} Q^{\bar{c}} V^{\bar{d}}, \\
& \left(-i g-h^{3}\right)_{\bar{a} \bar{b}}\left(i g+h^{3}\right)_{\bar{f} \bar{e}}\left(d^{1}+i d^{2}\right)_{\bar{c} \bar{g}} f_{d}^{\bar{f} \bar{g}} f_{{ }_{\bar{h}}}^{d \bar{b}} Q^{\bar{e}} Q^{\bar{c}} Q^{\bar{a}} V^{\bar{h}}= \\
& \quad\left[\left(h^{1}+i h^{2}\right)_{\bar{a} \bar{b}}\left(d^{0}-i d^{3}\right)_{\bar{c} \bar{d}}+2 i h_{\bar{a} \bar{b}}^{3}\left(d^{1}+i d^{2}\right)_{\bar{c} \bar{d}}-2 d_{\bar{a} \bar{b}}^{0}\left(h^{1}+i h^{2}\right)_{\bar{c} \bar{d}}\right] Q^{\bar{a}} Q^{\bar{b}} Q^{\bar{c}} V^{\bar{d}}, \\
& \left(i g+h^{3}\right)_{\bar{a} \bar{b}}\left(h^{1}-i h^{2}\right)_{\bar{e} \bar{f}}\left(d^{1}+i d^{2}\right)_{\bar{c} \bar{g}} f_{d}^{\bar{f} \bar{g}} f_{\bar{h}}^{d \bar{b}} Q^{\bar{e}} Q^{\bar{c}} Q^{\bar{a}} V^{\bar{h}}= \\
& \quad\left[\left(i h^{1}+h^{2}\right)_{\bar{a} \bar{b}}\left(d^{1}+i d^{2}\right)_{\bar{c} \bar{d}}+2 d_{\bar{a} \bar{b}}^{0}\left(i g+h^{3}\right)_{\bar{c} \bar{d}}\right] Q^{\bar{a}} Q^{\bar{b}} Q^{\bar{c}} V^{\bar{d}}, \\
& \left(i g-h^{3}\right)_{\bar{a} \bar{b}}\left(h^{1}-i h^{2}\right)_{\bar{e} \bar{f}}\left(d^{1}+i d^{2}\right)_{\bar{c} \bar{g}} f_{d}^{\bar{f} \bar{g}} f_{h}^{d \bar{h}} Q^{\bar{e}} Q^{\bar{c}} Q^{\bar{a}} V^{\bar{h}}= \\
& \quad-\left[\left(h^{1}+i h^{2}\right)_{\bar{a} \bar{b}}\left(i d^{1}+d^{2}\right)_{\bar{c} \bar{d}}+2 d_{\bar{a} \bar{b}}^{0}\left(i g-h^{3}\right)_{\bar{c} \bar{d}}\right] Q^{\bar{a}} Q^{\bar{b}} Q^{\bar{c}} V^{\bar{d}} .
\end{aligned}
$$

Again the first relation in (F.3) comes from the equation (3.46). The spin- $\frac{3}{2}$ current $\hat{G}_{21}(z)$ has a term $\left(h^{3}+i g\right)_{\bar{a} \bar{b}}$ and the higher spin-2 current has $\left(h^{1}-i h^{2}\right)_{\bar{e} \bar{f}}\left(i d^{0}-d^{3}\right)_{\bar{c} \bar{g}} f_{d}^{\bar{f} \bar{g}}$ in the cubic term of (3.27). Moreover, according to the third equation in (F.1), the last term has a quartic term. Combining these factors leads to the left hand side of the first equation of (F.3). The right hand side appears in the final expression of the higher spin- $\frac{5}{2}$ current given in (3.46).

\section{G Some OPEs generating the higher spin currents (for $N=3,5,7,9$ )}

Let us collect some relevant OPEs, (4.9) (4.13), (4.20) and (4.34) of [40], which generate the higher spin- $\frac{3}{2}$ currents. One can check they are the same for general $N$ from $(2.7),(2.16)$ and (3.13). Therefore, one has

$$
\begin{aligned}
& \hat{G}_{21}(z) T^{(1)}(w)=\frac{1}{(z-w)}\left[\hat{G}_{21}+2 T_{+}^{\left(\frac{3}{2}\right)}\right](w)+\cdots, \\
& \hat{G}_{12}(z) T^{(1)}(w)=\frac{1}{(z-w)}\left[-\hat{G}_{12}+2 T_{-}^{\left(\frac{3}{2}\right)}\right](w)+\cdots, \\
& \hat{G}_{11}(z) T^{(1)}(w)=\frac{1}{(z-w)}\left[\hat{G}_{11}+2 U^{\left(\frac{3}{2}\right)}\right](w)+\cdots, \\
& \hat{G}_{22}(z) T^{(1)}(w)=\frac{1}{(z-w)}\left[-\hat{G}_{22}+2 V^{\left(\frac{3}{2}\right)}\right](w)+\cdots .
\end{aligned}
$$


These OPEs (G.1) are used in subsection 3.2. One can also express these OPEs in the $\mathrm{SO}(4)$ representation using (2.16) in order to calculate (3.14).

Let us describe how one can generate the next higher spin-2 currents. In (2.7) and (2.16), the spin- $\frac{3}{2}$ currents in the bispinor notation are determined and the higher spin- $\frac{3}{2}$ currents are given in (3.15). Then one has

$$
\begin{aligned}
\hat{G}_{11}(z) \hat{G}_{12}^{\prime}(w)= & \frac{1}{(z-w)}\left[-2 U_{-}^{(2)}-\frac{4}{(N+k+2)} \hat{A}_{+} B_{3}\right](w)+\cdots, \\
\hat{G}_{21}(z) \hat{G}_{22}^{\prime}(w)= & \frac{1}{(z-w)}\left[2 V_{+}^{(2)}-\frac{4}{(N+k+2)} \hat{A}_{-} B_{3}\right](w)+\cdots \\
\hat{G}_{11}(z) \hat{G}_{21}^{\prime}(w)= & \frac{1}{(z-w)}\left[-2 U_{+}^{(2)}-\frac{4}{(N+k+2)} \hat{A}_{3} \hat{B}_{-}\right](w)+\cdots, \\
\hat{G}_{12}(z) \hat{G}_{22}^{\prime}(w)= & \frac{1}{(z-w)}\left[2 V_{-}^{(2)}-\frac{4}{(N+k+2)} \hat{A}_{3} \hat{B}_{+}\right](w)+\cdots, \\
\hat{G}_{12}(z) \hat{G}_{21}^{\prime}(w)= & \frac{1}{(z-w)^{2}} 2 T^{(1)}(w)+\frac{1}{(z-w)}\left[-2 T^{(2)}+\partial T^{(1)}+\frac{2(k+N)}{(k+N+2 k N)} \hat{T}\right. \\
& \left.+\frac{2}{(N+k+2)}\left(\hat{A}_{i} \hat{A}_{i}+\hat{B}_{i} \hat{B}_{i}-2 \hat{A}_{3} \hat{B}_{3}\right)\right](w)+\cdots, \\
\hat{G}_{11}(z) \hat{G}_{22}^{\prime}(w)= & \frac{1}{(z-w)^{2}} 2 T^{(1)}(w)+\frac{1}{(z-w)}\left[2 W^{(2)}+\partial T^{(1)}-2 \hat{T}\right. \\
& \left.-\frac{2}{(N+k+2)}\left(\hat{A}_{i} \hat{A}_{i}+\hat{B}_{i} \hat{B}_{i}+2 \hat{A}_{3} \hat{B}_{3}\right)\right](w)+\cdots, \\
\hat{G}_{21}(z) \hat{G}_{12}^{\prime}(w)= & \frac{1}{(z-w)^{2}} 2 T^{(1)}(w)+\frac{1}{(z-w)}\left[2 T^{(2)}+\partial T^{(1)}-\frac{2(k+N)}{(k+N+2 k N)} \hat{T}\right. \\
& \left.-\frac{2}{(N+k+2)}\left(\hat{A}_{i} \hat{A}_{i}+\hat{B}_{i} \hat{B}_{i}-2 \hat{A}_{3} \hat{B}_{3}\right)\right](w)+\cdots, \\
& \left.+\frac{2}{(N+k+2)}\left(\hat{A}_{i} \hat{A}_{i}+\hat{B}_{i} \hat{B}_{i}+2 \hat{A}_{3} \hat{B}_{3}\right)\right](w)+\cdots \\
\hat{G}_{22}(z) \hat{G}_{11}^{\prime}(w)= & \frac{1}{(z-w)^{2}} 2 T^{(1)}(w)+\frac{1}{(z-w)}\left[-2 W^{(2)}+\partial T^{(1)}+2 \hat{T}\right. \\
& \frac{2}{(N+2)}
\end{aligned}
$$

These OPEs (G.2) correspond to the second equation of appendix A of [20] where the right hand side contains only linear terms. One can check that according to the analysis in the subsection 3.3 the nonlinear terms in (G.2) can be absorbed into the redefined higher spin-2 currents. One has also following relations concerning on the singular terms

$$
\begin{aligned}
& \hat{G}_{12}(z) \hat{G}_{11}^{\prime}(w)=-\hat{G}_{11}(z) \hat{G}_{12}^{\prime}(w), \\
& \hat{G}_{22}(z) \hat{G}_{21}^{\prime}(w)=-\hat{G}_{21}(z) \hat{G}_{22}^{\prime}(w), \\
& \hat{G}_{21}(z) \hat{G}_{11}^{\prime}(w)=-\hat{G}_{11}(z) \hat{G}_{21}^{\prime}(w), \\
& \hat{G}_{22}(z) \hat{G}_{12}^{\prime}(w)=-\hat{G}_{12}(z) \hat{G}_{22}^{\prime}(w) .
\end{aligned}
$$

These OPEs (G.2) and (G.3) are used in subsection 3.3. The OPEs $G_{m n}^{\prime}(z) G_{p q}^{\prime}(w)$ can be obtained from the relation (3.17) together with (D.1), (3.18) and (2.16). 
The (4.31) (4.45), (4.52) and (4.55) of [40], which generate the higher spin- $\frac{5}{2}$ current can be generalized for general $N$ from (2.7), (2.16), (3.28), (3.32) and (3.34). Therefore, one obtains

$$
\begin{aligned}
\hat{G}_{21}(z) U_{-}^{(2)}(w)= & \frac{1}{(z-w)^{2}}\left[\frac{(N+2 k)}{(N+k+2)} \hat{G}_{11}+\frac{2(N+2 k+1)}{(N+k+2)} U^{\left(\frac{3}{2}\right)}\right](w) \\
& +\frac{1}{(z-w)}\left[U^{\left(\frac{5}{2}\right)}+\frac{1}{3} \partial\left\{\hat{G}_{21} U_{-}^{(2)}\right\}_{-2}\right](w)+\cdots \\
\hat{G}_{21}(z) V_{-}^{(2)}(w)= & \frac{1}{(z-w)^{2}}\left[-\frac{(2 N+k)}{(N+k+2)} \hat{G}_{22}+\frac{2(2 N+k+1)}{(N+k+2)} V^{\left(\frac{3}{2}\right)}\right](w) \\
& +\frac{1}{(z-w)}\left[V^{\left(\frac{5}{2}\right)}+\frac{1}{3} \partial\left\{\hat{G}_{21} V_{-}^{(2)}\right\}_{-2}\right](w)+\cdots \\
\hat{G}_{21}(z) W^{(2)}(w)= & \frac{1}{(z-w)^{2}}\left[\frac{(N+2 k+1)}{(N+k+2)} \hat{G}_{21}+\frac{(k-N)}{(N+k+2)} T_{+}^{\left(\frac{3}{2}\right)}\right](w) \\
& +\frac{1}{(z-w)}\left[W_{+}^{\left(\frac{5}{2}\right)}+\frac{1}{3} \partial\left\{\hat{G}_{21} W^{(2)}\right\}_{-2}\right](w)+\cdots \\
\hat{G}_{12}(z) W^{(2)}(w)= & \frac{1}{(z-w)^{2}}\left[\frac{(N+2 k+1)}{(N+k+2)} \hat{G}_{12}+\frac{(N-k)}{(N+k+2)} T_{-}^{\left(\frac{3}{2}\right)}\right](w) \\
& +\frac{1}{(z-w)}\left[W_{-}^{\left(\frac{5}{2}\right)}+\frac{1}{3} \partial\left\{\hat{G}_{12} W^{(2)}\right\}_{-2}\right](w)+\cdots
\end{aligned}
$$

These OPEs (G.4) are used in subsection 3.4.

\section{H The coefficients and some OPEs relevant to the higher spin-3 current}

Furthermore, the equation (4.59) of [40] can be generalized to the equation (3.49) and the $(N, k)$ dependent coefficients are given by (for $N=3,5,7,9)$

$$
\begin{aligned}
& a_{1}=\frac{8 i N(3 k+1)}{3(N+k+2)^{2}}, \\
& a_{2}=\frac{8 i k(3 N+1)}{3(N+k+2)^{2}}, \\
& a_{3}=\frac{8(k-N)}{3(N+k+2)}, \\
& a_{4}=-\frac{4\left[\left(2 N^{2}-N\right)+\left(6 N^{2}+4 N-1\right) k+(6 N+4) k^{2}\right]}{3(N+k+2)(N+k+2 k N)}, \\
& a_{5}=\frac{4(k-N)}{3(N+k+2)}, \\
& a_{6}=\frac{4(N+k+1)}{(N+k+2)}, \\
& a_{7}=-\frac{4(-N+4 k-1)}{3(N+k+2)^{2}}, \\
& a_{8}=-\frac{4(-4 N+4 k-1)}{3(N+k+2)^{2}},
\end{aligned}
$$




$$
\begin{aligned}
a_{9} & =\frac{8(-N+k-1)}{3(N+k+2)^{2}}, \\
a_{10} & =-\frac{4(2 N+k-1)}{3(N+k+2)^{2}}, \\
a_{11} & =\frac{4(-2 N+2 k+1)}{3(N+k+2)^{2}}, \\
a_{12} & =-\frac{4 i}{(N+k+2)}, \\
a_{13} & =\frac{8 i N(3 k+1)}{(N+k+2)(5 N+4+(6 N+5) k)}, \\
a_{14} & =\frac{8 i k(3 N+1)}{(N+k+2)(5 N+4+(6 N+5) k)}, \\
a_{15} & =\frac{8(k-N)}{(5 N+4+(6 N+5) k)} .
\end{aligned}
$$

The numerators of these coefficients have a simple linear in $N$ except $a_{4}$. For the higher spin- 3 current, one should calculate the spin- $\frac{3}{2}$ current and the spin- $\frac{5}{2}$ current. The former has $Q^{\bar{a}} V^{\bar{b}}(z)$ and the latter contains the two quadratic terms, the cubic term and the quartic term.

It turns out that the first order poles of the corresponding OPEs are given by

$$
\begin{aligned}
& \left.Q^{\bar{a}} V^{\bar{b}}(z) \partial Q^{\bar{c}} V^{\bar{d}}(w)\right|_{\frac{1}{z-w}}=\left[(k+N+2)\left(-g^{\bar{c} \bar{a}} V^{\bar{d}} \partial V^{\bar{b}}+\frac{1}{2} g^{\bar{c} \bar{a}} f_{e}^{\bar{b} \bar{d}} \partial^{2} V^{e}\right)\right. \\
& \left.-k g^{\bar{d} \bar{b}} \partial Q^{\bar{c}} \partial Q^{\bar{a}}-f_{e}^{\bar{b} \bar{d}} Q^{\bar{a}} \partial Q^{\bar{c}} V^{e}\right](w), \\
& \left.Q^{\bar{a}} V^{\bar{b}}(z) Q^{\bar{c}} \partial V^{\bar{d}}(w)\right|_{\frac{1}{z-w}}=\left[-(k+N+2) g^{\bar{c} \bar{a}} V^{\bar{b}} \partial V^{\bar{d}}-k g^{\bar{d} b} Q^{\bar{c}} \partial^{2} Q^{\bar{a}}\right. \\
& \left.-f_{e}^{\bar{d} \bar{b}} Q^{\bar{c}} \partial\left(Q^{\bar{a}} V^{e}\right)\right](w) \\
& \left.Q^{\bar{a}} V^{\bar{b}}(z) Q^{\bar{c}} V^{\bar{d}} V^{e}(w)\right|_{\frac{1}{z-w}}=\left[-(k+N+2) g^{\bar{c} \bar{a}} V^{\bar{b}} V^{\bar{d}} V^{e}-k g^{\overline{d \bar{b}}} Q^{\bar{c}} \partial Q^{\bar{a}} V^{e}\right. \\
& -f_{g}^{\overline{d \bar{b}}} Q^{\bar{c}} Q^{\bar{a}} V^{e} V^{g}-f_{g}^{\overline{d b}} f_{h}^{e g} Q^{\bar{c}} \partial\left(Q^{\bar{a}} V^{h}\right)-f_{g}^{e \bar{b}} Q^{\bar{c}} Q^{\bar{a}} V^{\bar{d}} V^{g} \\
& \left.-\frac{1}{2} k f_{g}^{\overline{d b}} g^{e g} Q^{\bar{c}} \partial^{2} Q^{\bar{a}}-k g^{e \bar{b}} Q^{\bar{c}} \partial Q^{\bar{a}} V^{\bar{d}}\right](w), \\
& \left.Q^{\bar{a}} V^{\bar{b}}(z) Q^{\bar{c}} Q^{\bar{d}} Q^{\bar{e}} V^{f}(w)\right|_{\frac{1}{z-w}}=\left[( k + N + 2 ) \left(g^{\bar{e} \bar{a}} Q^{\bar{d}} Q^{\bar{c}} V^{\bar{b}} V^{f}-g^{\bar{d} \bar{a}} Q^{\bar{e}} Q^{\bar{c}} V^{\bar{b}} V^{f}\right.\right. \\
& \left.+g^{\bar{c} \bar{a}} Q^{\bar{e}} Q^{\bar{d}} V^{\bar{b}} V^{f}\right)+f_{g}^{f \bar{b}} Q^{\bar{e}} Q^{\bar{d}} Q^{\bar{c}} Q^{\bar{a}} V^{g} \\
& \left.+k g^{f \bar{b}} Q^{\bar{e}} Q^{\bar{d}} Q^{\bar{c}} \partial Q^{\bar{a}}\right](w) \text {. }
\end{aligned}
$$

Then, the final higher spin-3 current can be obtained by multiplying $h_{\bar{a} \bar{b}}^{\mu}$ and other relevant rank two, three and four tensors (appearing in the higher spin- $\frac{5}{2}$ current) in (H.2). The spin-3 current have the composite terms from the currents of large $\mathcal{N}=4$ nonlinear superconformal algebra. One obtains the contributions from the OPEs between the spin- $\frac{3}{2}$ current and those composite terms without any difficulty as before. 
Open Access. This article is distributed under the terms of the Creative Commons Attribution License (CC-BY 4.0), which permits any use, distribution and reproduction in any medium, provided the original author(s) and source are credited.

\section{References}

[1] M.R. Gaberdiel and R. Gopakumar, An AdS 3 Dual for Minimal Model CFTs, Phys. Rev. D 83 (2011) 066007 [arXiv: 1011.2986] [INSPIRE].

[2] M.R. Gaberdiel and R. Gopakumar, Triality in Minimal Model Holography, JHEP 07 (2012) 127 [arXiv:1205.2472] [INSPIRE].

[3] M.R. Gaberdiel and R. Gopakumar, Minimal Model Holography, J. Phys. A 46 (2013) 214002 [arXiv:1207.6697] [InSPIRE].

[4] M.R. Gaberdiel and R. Gopakumar, Large- $\mathcal{N}=4$ Holography, JHEP 09 (2013) 036 [arXiv: 1305.4181] [INSPIRE].

[5] J.A. Wolf, Complex Homogeneous Contact Manifolds and Quaternionic Symmetric Spaces, J. Math. Mech. 14 (1965) 1033.

[6] D.V. Alekseevskii, Classification of Quarternionic Spaces with a Transitive Solvable Group of Motions, Math. USSR Izv. 9 (1975) 297.

[7] S. Salamon, Quaternionic Kahler Manifolds, Invent. Math. 67 (1982) 143.

[8] T. Creutzig, Y. Hikida and P.B. Ronne, Extended higher spin holography and Grassmannian models, JHEP 11 (2013) 038 [arXiv:1306.0466] [INSPIRE].

[9] T. Creutzig, Y. Hikida and P.B. Ronne, Higher spin $A d S_{3}$ supergravity and its dual CFT, JHEP 02 (2012) 109 [arXiv:1111.2139] [INSPIRE].

[10] Y. Kazama and H. Suzuki, New $N=2$ Superconformal Field Theories and Superstring Compactification, Nucl. Phys. B 321 (1989) 232 [INSPIRE].

[11] Y. Kazama and H. Suzuki, Characterization of $N=2$ Superconformal Models Generated by Coset Space Method, Phys. Lett. B 216 (1989) 112 [INSPIRE].

[12] C. Candu and M.R. Gaberdiel, Supersymmetric holography on AdS $S_{3}$, JHEP 09 (2013) 071 [arXiv: 1203.1939] [INSPIRE].

[13] K. Hanaki and C. Peng, Symmetries of Holographic Super-Minimal Models, JHEP 08 (2013) 030 [arXiv:1203.5768] [INSPIRE].

[14] C. Ahn, The Large-N 't Hooft Limit of Kazama-Suzuki Model, JHEP 08 (2012) 047 [arXiv:1206.0054] [INSPIRE].

[15] C. Candu and M.R. Gaberdiel, Duality in $N=2$ Minimal Model Holography, JHEP 02 (2013) 070 [arXiv:1207.6646] [INSPIRE].

[16] C. Ahn, The Operator Product Expansion of the Lowest Higher Spin Current at Finite N, JHEP 01 (2013) 041 [arXiv: 1208.0058] [INSPIRE].

[17] Y. Hikida, Conical defects and $N=2$ higher spin holography, JHEP 08 (2013) 127 [arXiv: 1212.4124] [INSPIRE].

[18] C. Candu, C. Peng and C. Vollenweider, Extended supersymmetry in AdS $S_{3}$ higher spin theories, arXiv:1408.5144 [INSPIRE]. 
[19] C. Candu and C. Vollenweider, On the coset duals of extended higher spin theories, JHEP 04 (2014) 145 [arXiv: 1312.5240] [INSPIRE].

[20] M. Beccaria, C. Candu and M.R. Gaberdiel, The large- $N=4$ superconformal $W_{\infty}$ algebra, JHEP 06 (2014) 117 [arXiv:1404.1694] [INSPIRE].

[21] M.R. Gaberdiel and R. Gopakumar, Higher Spins \& Strings, JHEP 11 (2014) 044 [arXiv: 1406.6103] [INSPIRE].

[22] T. Creutzig, Y. Hikida and P.B. Ronne, Higher spin AdS $S_{3}$ holography with extended supersymmetry, JHEP 10 (2014) 163 [arXiv:1406.1521] [INSPIRE].

[23] C. Ahn, The Higher Spin Currents in the $N=1$ Stringy Coset Minimal Model, JHEP 04 (2013) 033 [arXiv: 1211.2589] [InSPIRE].

[24] C. Ahn, Higher Spin Currents with Arbitrary $N$ in the $\mathcal{N}=1$ Stringy Coset Minimal Model, JHEP 07 (2013) 141 [arXiv: 1305.5892] [INSPIRE].

[25] M. Beccaria, C. Candu, M.R. Gaberdiel and M. Groher, $\mathcal{N}=1$ extension of minimal model holography, arXiv:1305.1048 [INSPIRE].

[26] P. Goddard and A. Schwimmer, Factoring Out Free Fermions and Superconformal Algebras, Phys. Lett. B 214 (1988) 209 [INSPIRE].

[27] A. Van Proeyen, Realizations of $N=4$ Superconformal Algebras on Wolf Spaces, Class. Quant. Grav. 6 (1989) 1501 [INSPIRE].

[28] M. Günaydin, J.L. Petersen, A. Taormina and A. Van Proeyen, On the Unitary Representations of a Class of $N=4$ Superconformal Algebras, Nucl. Phys. B 322 (1989) 402 [INSPIRE].

[29] S.J. Gates Jr. and S.V. Ketov, No $N=4$ strings on wolf spaces, Phys. Rev. D 52 (1995) 2278 [hep-th/9501140] [INSPIRE].

[30] M. Günaydin, $N=4$ superconformal algebras and gauged WZW models, Phys. Rev. D 47 (1993) 3600 [hep-th/9301049] [INSPIRE].

[31] A. Sevrin, W. Troost and A. Van Proeyen, Superconformal Algebras in Two-Dimensions with $N=4$, Phys. Lett. B 208 (1988) 447 [INSPIRE].

[32] A. Sevrin, W. Troost, A. Van Proeyen and P. Spindel, Extended supersymmetric $\sigma$-models on group manifolds. 2. Current algebras, Nucl. Phys. B 311 (1988) 465 [INSPIRE].

[33] K. Schoutens, $O(n)$ Extended Superconformal Field Theory in Superspace, Nucl. Phys. B 295 (1988) 634 [inSPIRE].

[34] E.A. Ivanov and S.O. Krivonos, $N=4$ superLiouville equation (in Russian), J. Phys. A 17 (1984) L671 [inSPIRE].

[35] E.A. Ivanov and S.O. Krivonos, $N=4$ Superextension of the Liouville Equation With Quaternionic Structure, Theor. Math. Phys. 63 (1985) 477 [INSPIRE].

[36] E.A. Ivanov, S.O. Krivonos and V.M. Leviant, A New Class of Superconformal $\sigma$ Models With the Wess-Zumino Action, Nucl. Phys. B 304 (1988) 601 [INSPIRE].

[37] E.A. Ivanov, S.O. Krivonos and V.M. Leviant, Quantum $N=3, N=4$ Superconformal WZW $\sigma$-models, Phys. Lett. B 215 (1988) 689 [Erratum ibid. B 221 (1989) 432] [INSPIRE].

[38] A. Sevrin and G. Theodoridis, $N=4$ superconformal coset theories, Nucl. Phys. B 332 (1990) 380 [inSPIRE]. 
[39] N. Saulina, Geometric interpretation of the large-N $=4$ index, Nucl. Phys. B 706 (2005) 491 [hep-th/0409175] [INSPIRE].

[40] C. Ahn, Higher Spin Currents in Wolf Space. Part I, JHEP 03 (2014) 091 [arXiv:1311.6205] [INSPIRE].

[41] C. Ahn, Higher Spin Currents in Wolf Space. Part II, arXiv:1408.0655 [InSPIRE].

[42] K. Thielemans, A Mathematica package for computing operator product expansions, Int. J. Mod. Phys. C 2 (1991) 787 [InSPIRE].

[43] V.G. Kac and I.T. Todorov, Superconformal current algebras and their unitary representations, Commun. Math. Phys. 102 (1985) 337 [INSPIRE].

[44] C.M. Hull and B.J. Spence, $N=2$ Current Algebra and Coset Models, Phys. Lett. B 241 (1990) 357 [inSPIRE].

[45] F.A. Bais, P. Bouwknegt, M. Surridge and K. Schoutens, Extensions of the Virasoro Algebra Constructed from Kac-Moody Algebras Using Higher Order Casimir Invariants, Nucl. Phys. B 304 (1988) 348 [InSPIRE].

[46] F.A. Bais, P. Bouwknegt, M. Surridge and K. Schoutens, Coset Construction for Extended Virasoro Algebras, Nucl. Phys. B 304 (1988) 371 [INSPIRE].

[47] P. Bouwknegt and K. Schoutens, W symmetry in conformal field theory, Phys. Rept. 223 (1993) 183 [hep-th/9210010] [INSPIRE].

[48] J. Michelson and A. Strominger, The Geometry of (super)conformal quantum mechanics, Commun. Math. Phys. 213 (2000) 1 [hep-th/9907191] [INSPIRE].

[49] M.R. Gaberdiel and C. Peng, The symmetry of large $\mathcal{N}=4$ holography, JHEP 05 (2014) 152 [arXiv: 1403.2396] [INSPIRE].

[50] M.R. Gaberdiel and T. Hartman, Symmetries of Holographic Minimal Models, JHEP 05 (2011) 031 [arXiv:1101.2910] [INSPIRE].

[51] C. Ahn, The Coset Spin-4 Casimir Operator and Its Three-Point Functions with Scalars, JHEP 02 (2012) 027 [arXiv:1111.0091] [INSPIRE].

[52] T. Creutzig, Y. Hikida and P.B. Ronne, Three point functions in higher spin $A d S_{3}$ supergravity, JHEP 01 (2013) 171 [arXiv:1211.2237] [INSPIRE].

[53] H. Moradi and K. Zoubos, Three-Point Functions in $N=2$ Higher-Spin Holography, JHEP 04 (2013) 018 [arXiv:1211.2239] [INSPIRE].

[54] C. Ahn and H. Kim, Spin-5 Casimir operator its three-point functions with two scalars, JHEP 01 (2014) 012 [Erratum ibid. 1401 (2014) 174] [arXiv:1308.1726] [INSPIRE].

[55] C. Ahn, The Large-N 't Hooft Limit of Coset Minimal Models, JHEP 10 (2011) 125 [arXiv: 1106.0351] [INSPIRE].

[56] M.R. Gaberdiel and C. Vollenweider, Minimal Model Holography for $\mathrm{SO}(2 N)$, JHEP 08 (2011) 104 [arXiv:1106.2634] [INSPIRE].

[57] C. Ahn, The Primary Spin-4 Casimir Operators in the Holographic $\mathrm{SO}(N)$ Coset Minimal Models, JHEP 05 (2012) 040 [arXiv:1202.0074] [INSPIRE].

[58] T. Creutzig, Y. Hikida and P.B. Rønne, $N=1$ supersymmetric higher spin holography on $A d S_{3}, J H E P 02(2013) 019$ [arXiv: 1209.5404] [INSPIRE]. 
[59] C. Candu, M.R. Gaberdiel, M. Kelm and C. Vollenweider, Even spin minimal model holography, JHEP 01 (2013) 185 [arXiv:1211.3113] [INSPIRE].

[60] C. Ahn and J. Paeng, The OPEs of Spin-4 Casimir Currents in the Holographic $\mathrm{SO}(N)$ Coset Minimal Models, Class. Quant. Grav. 30 (2013) 175004 [arXiv:1301.0208] [InSPIRE].

[61] C. Ahn and J. Paeng, Higher Spin Currents in the Holographic $\mathcal{N}=1$ Coset Minimal Model, JHEP 01 (2014) 007 [arXiv:1310.6185] [INSPIRE].

[62] C. Ahn and J. Paeng, Higher Spin Currents in Orthogonal Wolf Space, arXiv:1410.0080 [INSPIRE].

[63] S.F. Prokushkin and M.A. Vasiliev, Higher spin gauge interactions for massive matter fields in 3 -D AdS space-time, Nucl. Phys. B 545 (1999) 385 [hep-th/9806236] [InSPIRE].

[64] S. Prokushkin and M.A. Vasiliev, $3-D$ higher spin gauge theories with matter, hep-th/9812242 [INSPIRE].

[65] H. Georgi, Frontiers in Physics. Vol. 54: Lie Algebras In Particle Physics. From Isospin To Unified Theories, second edition, Westview Press, Boulder U.S.A. (1999). 